\title{
Coulomb and Higgs branches from canonical singularities. Part 0
}

\author{
Cyril Closset, Sakura Schäfer-Nameki and Yi-Nan Wang \\ Mathematical Institute, University of Oxford, \\ Andrew-Wiles Building, Woodstock Road, Oxford, OX2 6GG, U.K. \\ E-mail: cyril.closset@maths.ox.ac.uk
}

ABSTRACT: Five- and four-dimensional superconformal field theories with eight supercharges arise from canonical threefold singularities in M-theory and Type IIB string theory, respectively. We study their Coulomb and Higgs branches using crepant resolutions and deformations of the singularities. We propose a relation between the resulting moduli spaces, by compactifying the theories to $3 \mathrm{~d}$, followed by $3 \mathrm{~d} \mathcal{N}=4$ mirror symmetry and an $S$-type gauging of an abelian flavor symmetry. In particular, we use this correspondence to determine the Higgs branch of some 5d SCFTs and their magnetic quivers from the geometry. As an application of the general framework, we observe that singularities that engineer Argyres-Douglas theories in Type IIB also give rise to rank-0 5d SCFTs in M-theory. We also compute the higher-form symmetries of the 4d and 5d SCFTs, including the one-form symmetries of generalized Argyres-Douglas theories of type $\left(G, G^{\prime}\right)$.

Keywords: Field Theories in Higher Dimensions, Supersymmetric Gauge Theory, MTheory, String Duality

ARXIV EPRINT: 2007.15600 


\section{Contents}

1 Introduction 1

1.1 Setup and summary 2

1.2 Comments on rank-0 theories from singularities 5

$\begin{array}{llll}2 & 5 \mathrm{~d} / 4 \mathrm{~d} \text { correspondence via } 3 \mathrm{~d} \text { mirror symmetry } & 6\end{array}$

$2.15 \mathrm{~d}$ SCFTs from M-theory 6

2.2 4d SCFTs from Type IIB 8

2.3 Crepant resolution and anomaly matching on the Higgs branch of $\mathscr{T}_{\mathbf{X}}^{4 \mathrm{~d}} \quad 10$

2.4 Flavor symmetries, conifold transitions and boundary five-manifold 11

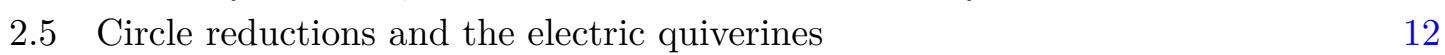

2.6 Higgs branches, magnetic quiverines and 3d mirrors 14

$\begin{array}{lll}2.7 & \text { Higher-form symmetries of SCFTs } & 16\end{array}$

3 5d SCFTs with Lagrangian 4d SCFT counterparts $\quad 17$

$\begin{array}{lll}3.1 & \text { The rank- } N E_{8} \text { theory } & 18\end{array}$

$\begin{array}{lll}3.1 .1 & \text { Singularity spectrum and 4d superconformal quiver } & 18\end{array}$

3.1.2 Resolutions: 5d Coulomb branch and 4d Higgs branch 20

3.1.3 Magnetic quivers and the 5d Higgs branch 23

3.2 Rank-two 5d SCFT with a gauge-theory phase $\mathrm{SU}(2)_{0}-\mathrm{SU}(2)-5 \mathbf{F} \quad 23$

3.2.1 Singularity spectrum and 4d superconformal quiver 24

3.2.2 Resolved geometry and 5d Coulomb branch 25

3.3 Rank-two 5d SCFT with a gauge-theory phase $G_{2}+5 \mathbf{F} \quad 26$

$\begin{array}{lll}\text { 3.3.1 Singularity spectrum and 4d superconformal quiver } & 26\end{array}$

$\begin{array}{ll}\text { 3.3.2 Resolved geometry and 5d Coulomb branch } & 27\end{array}$

4 Argyres-Douglas theories and their 5d SCFT counterparts 29

4.14 d fixed-points with $r=0$ and 3d mirrors 29

$\begin{array}{ll}4.25 \mathrm{~d} \text { rank-zero fixed points and their magnetic quivers } & 32\end{array}$

$\begin{array}{lll}4.3 & \text { Further examples: higher-rank 5d theories } & 34\end{array}$

4.3.1 The $\mathbf{X}_{\left[A_{2}, D_{4}\right]}$ theory 34

4.3.2 Example of a rank-one 5d SCFT coupled to a rank-zero theory 35

4.4 One-form symmetries of the 4d SCFTs $\operatorname{AD}\left[G, G^{\prime}\right] \quad 36$

5 Rank-zero 4d SCFTs from isolated toric singularities 38

$\begin{array}{lll}5.1 & \text { Toric singularities and deformations } & 38\end{array}$

5.2 The $Y^{N, 0}$ geometry, $5 \mathrm{~d} \mathrm{SU}(N)_{0}$ and rank-zero 4d SCFTs 39

A Higgs-branch Hilbert series from magnetic quivers $\quad 42$

A.1 The $\mathrm{CB}$ of $\mathrm{EQ}^{(4)}$ for $\mathrm{AD}\left[A_{2}, D_{4}\right] \quad 44$

A.2 Gauging the affine $\widehat{a}_{N-1}$ quiver 44 


\section{Introduction}

Many supersymmetric quantum field theories (SQFT) can be realized within string theory, which provides us with powerful tools to study them in a strongly coupled regime, often in terms of geometry [1-8]. In this paper, we take yet another look at the 'geometric engineering' of superconformal field theories (SCFT) in space-time dimension $d=4$ and $d=5$. Our leitmotiv will be that one can refine our understanding of these systems, in both $4 \mathrm{~d}$ and $5 \mathrm{~d}$, by considering their relations to each other via toroidal compactification to 3d [9]. A more detailed description of our geometric approach, together with many examples, will appear in a forthcoming paper [10].

These theories are partly characterized by their moduli space of vacua, which generally includes both a Coulomb and a Higgs branch, together with mixed branches. In the case of 5d SCFTs, their moduli spaces can be studied from their geometric realization in M-theory on a canonical singularity: the crepant (i.e. retaining the Calabi-Yau condition) resolutions model the Coulomb branch, whereas the Higgs branch corresponds to the deformations of the singularity. The former has recently been utilized in exploring the classification of 5d SCFTs in [11-28]. On the Higgs branch side, the approach using brane-webs [29] has been particularly successful [30-38]. In the latter approach, one constructs the so-called magnetic quiver from a IIB brane web, a $3 \mathrm{~d} \mathcal{N}=4$ gauge theory whose Coulomb branch is conjectured to be the identical to the 5d Higgs branch.

So far, the analysis of the $5 \mathrm{~d}$ Higgs branch directly from the canonical-singularity point of view has been lagging behind. This is partly because the low-energy physics arising from M-theory on the deformed singularity is subject to M2-brane instanton corrections. In this paper and in [10], we explore the deformation theory of the hypersurface canonical singularities classified in [39] ${ }^{1}$ and we discuss the general structure of the Higgs branch for isolated hypersurface singularities. We concurrently explore the Coulomb branch, which arises from the crepant resolutions of such singularities. We also determine the higher-form symmetries of these $5 \mathrm{~d}$ theories, which provide further information about their spectrum of defect operators. The exploration of this vast class of models give us a wonderful playground to test current ideas about 5d SCFTs.

In this paper, we focus on a few interesting points, which deserve particular attention. First, we will argue that the magnetic quiver of the 5d SCFT is closely related to the $4 \mathrm{~d}$ SCFT that arises by geometric engineering in Type IIB string theory on the same singularity. (The $4 \mathrm{~d}$ geometric engineering in IIB has also studied in a lot detail over the years, see e.g. $[8,42-47]$.) This gives us a complementary understanding of magnetic quivers, and of

\footnotetext{
${ }^{1}$ See also [40]. For more mathematical background, see [41].
} 
their generalizations, directly in the geometric-engineering picture. The relation between the $5 \mathrm{~d}$ and $4 \mathrm{~d}$ SCFT goes through dimensional reduction to $3 \mathrm{~d}$, thanks to $3 \mathrm{~d} \mathcal{N}=4$ mirror symmetry [48], as first studied in [9]. In the IIB picture, M2-brane instantons become D3brane instantons wrapping a 3-cycle times a circle; these correspond to monopole operators in the $3 \mathrm{~d} \mathcal{N}=4$ magnetic-quiver theory, which can be more efficiently resummed [49-52].

Conversely, our analysis of the geometry of canonical hypersurface singularities allows us to also make statements about 4d SCFTs. For instance, we can study the Higgs branch of 4d SCFTs by studying the crepant resolutions of canonical singularities, and identify the additional low-energy degrees of freedom on the 4d Higgs branch (such as free vector multiplets and irreducibles SCFTs) from the resolution. Similarly, the Coulomb branch of the $4 \mathrm{~d}$ SCFT is closely related to the $5 \mathrm{~d}$ Higgs branch.

There has been a lot of recent progress in studying the $4 \mathrm{~d} \mathcal{N}=2$ Coulomb branch for a limited list of references see [53-56] — as well as the Higgs branch — see e.g. [57-67] and much of the SCFT/Vertex operator algebra literature [68], as in e.g. [69-71]. It would be greatly desirable to better understand how much of these beautiful structures can be recovered from the geometric-engineering framework.

Let us now outline the logical structure of the paper, and highlight some of the results.

\subsection{Setup and summary}

Consider a canonical threefold singularity $\mathbf{X}$. An important class of examples consists of the isolated hypersurface singularities (IHS). An IHS is defined by a single quasi-homogeneous polynomial $F(x)$ in $\mathbb{C}^{4}$ :

$$
\mathbf{X} \cong\left\{\left(x_{1}, x_{2}, x_{3}, x_{4}\right) \in \mathbb{C}^{4} \mid F\left(x_{1}, x_{2}, x_{3}, x_{4}\right)=0\right\},
$$

We require this space to be singular, i.e. $\partial_{x_{i}} F=F=0$ at an isolated point, with an additional condition so that $\mathbf{X}$ is canonical, as studied in M-theory e.g. in [72]. This will provide our main class of examples in this paper. ${ }^{2}$ The canonical singularity $\mathbf{X}$ in M-theory defines a $5 \mathrm{~d}$ SCFT, which we denote by $\mathcal{T}_{\mathbf{X}}^{5 \mathrm{~d}}$ :

$$
\mathcal{T}_{\mathbf{X}}^{5 \mathrm{~d}} \quad \longleftrightarrow \quad \text { M-theory on } \mathbb{R}^{1,4} \times \mathbf{X}
$$

while the same singularity in Type IIB string theory defines a $4 \mathrm{~d} \mathcal{N}=2$ SCFT, $\mathscr{T}_{\mathbf{X}}^{4 d}$ :

$$
\mathscr{T}_{\mathbf{X}}^{4 \mathrm{~d}} \quad \longleftrightarrow \quad \text { Type IIB on } \mathbb{R}^{1,3} \times \mathbf{X}
$$

These two superconformal field theories, in space-times of different dimensions, are of course distinct systems, but they are closely related. Consider compactifying the theory $\mathcal{T}_{\mathbf{X}}^{5 \mathrm{~d}}$ on a circle. We obtain a KK-theory in $4 \mathrm{~d}$ with $4 \mathrm{~d} \mathcal{N}=2$ supersymmetry, denoted by $D_{S^{1}} \mathcal{T}_{\mathbf{X}}^{5 \mathrm{~d}}$. By the M-theory/Type IIA duality, this theory is engineered by Type IIA string theory on $\mathbb{R}^{1,3} \times \mathbf{X}[1,73]$. If we further consider this theory on an arbitrary space-time manifold $\mathcal{M}_{4}$, we have

$$
D_{S^{1}} \mathcal{T}_{\mathbf{X}}^{5 \mathrm{~d}} \text { on } \mathcal{M}_{4} \quad \longleftrightarrow \quad \text { Type IIA on } \mathcal{M}_{4} \times \mathbf{X}
$$

\footnotetext{
${ }^{2}$ Another important class of examples, which we will also consider, consists of $\mathrm{CY}_{3}$ toric singularities.
} 
In particular, we may consider $\mathcal{M}_{4}=\mathbb{R}^{1,2} \times S_{\beta}^{1}$, corresponding to a toroidal compactification of $\mathcal{T}_{\mathbf{X}}^{5 \mathrm{~d}}$ on a circle of radius $\beta$. This SQFT has $3 \mathrm{~d} \mathcal{N}=4$ supersymmetry. Similarly, we may compactify the $4 \mathrm{~d}$ SCFT $\mathscr{T}_{\mathbf{X}}^{4 \mathrm{~d}}$ on a circle, to obtain another $3 \mathrm{~d}$ KK-theory with $\mathcal{N}=4$ supersymmetry. These two three-dimensional systems are related by T-duality

$$
\begin{aligned}
& D_{T^{2}} \mathcal{T}_{\mathbf{X}}^{5 \mathrm{~d}} \quad \longleftrightarrow \quad \text { M-theory on } \mathbb{R}^{1,2} \times T^{2} \times \mathbf{X} \\
& \longleftrightarrow \quad \text { Type IIA on } \mathbb{R}^{1,2} \times S_{\beta}^{1} \times \mathbf{X} \\
& \longleftrightarrow \quad \text { Type IIB on } \mathbb{R}^{1,2} \times S_{\frac{1}{\beta}}^{1} \times \mathbf{X} \quad \longleftrightarrow \quad D_{S^{1}} \mathscr{T}_{\mathbf{X}}^{4 \mathrm{~d}} \text {. }
\end{aligned}
$$

This implies the equivalence of two distinct-looking $3 \mathrm{~d} \mathcal{N}=4 \mathrm{KK}$-theories. In particular, the equivalence must hold in the extreme infrared (IR) limit (that is, decoupling all the KK-modes, by focussing on the low-energy limit at fixed $\beta$ ), which must give us a $3 \mathrm{~d} \mathcal{N}=4$ superconformal field theory. This IR SCFT will often have some useful description as the IR fixed point of an intrinsically $3 \mathrm{~d} \mathcal{N}=4 \mathrm{QFT}$, for instance as a $3 \mathrm{~d} \mathcal{N}=4$ gauge theory. Such 3d QFT descriptions may be derivable in the string theory engineering (1.5), or it may be more subtle (for instance requiring further string dualities) - in general, it may be a 'non-Lagrangian' theory, meaning that no useful UV Lagrangian in known in 3d. We will call these $3 \mathrm{~d} \mathcal{N}=4 \mathrm{SQFTs}$ the 'electric quiverine' (EQ) theories of the $5 \mathrm{~d}$ and $4 \mathrm{~d}$ SCFT, respectively, which we denote by

$$
\mathrm{EQ}^{(5)}[\mathbf{X}] \stackrel{3 \mathrm{~d}}{\cong} D_{T^{2}} \mathcal{T}_{\mathbf{X}}^{5 \mathrm{~d}}, \quad \quad \mathrm{EQ}^{(4)}[\mathbf{X}] \stackrel{3 \mathrm{~d}}{\cong} D_{S^{1}} \mathscr{T}_{\mathbf{X}}^{4 \mathrm{~d}}
$$

Here, the equivalence relation $\stackrel{3 \mathrm{~d}}{\cong}$ ' means that the two theories are equivalent as $3 \mathrm{~d} \mathcal{N}=4$ QFTs below the scale set by the lightest KK-modes. In many interesting cases, the quiverine has a description as a $3 \mathrm{~d} \mathcal{N}=4$ gauge-theory quiver, while in general it might not be a quiver, or not have any known Lagrangian description at all.

The T-duality (1.5) realizes $3 \mathrm{~d} \mathcal{N}=4$ mirror symmetry [9, 48], although not without an interesting subtlety. In general, the theories $\mathcal{T}_{\mathbf{X}}^{5 \mathrm{~d}}$ and $\mathscr{T}_{\mathbf{X}}^{4 \mathrm{~d}}$ have a $\mathrm{U}(1)^{f}$ symmetry acting on their Higgs branch (which is generally a subgroup of the full flavor symmetry $G_{H}^{5 \mathrm{~d}}$ and $G_{H}^{4 \mathrm{~d}}$, respectively). The M-theory and IIB realizations provide background gauge fields for this $\mathrm{U}(1)^{f}$ in the Coulomb-branch phase, coming from the reduction of the 3form gauge field $C_{3}$ on 2-cycles in M-theory, and from the RR field $C_{4}$ on 3-cycles in IIB, respectively. Upon toroidal compactification, these background abelian gauge fields may be effectively gauged in the $3 \mathrm{~d} \mathcal{N}=4$ description, depending on whether we are looking at the far IR limit of $D_{T^{2}} \mathcal{T}_{\mathbf{X}}^{5 \mathrm{~d}}$ or $D_{S^{1}} \mathscr{T}_{\mathbf{X}}^{4 \mathrm{~d}}$. Recall also that in $3 \mathrm{~d}$ one may gauge and 'ungauge' any $\mathrm{U}(1)$ current at minimal cost, and in a reversible manner - this is known as the ' $S$ operation' $[74,75]$, — because each gauged U(1) gives rise to a new topological current. Let us then define the following 'magnetic quiverine' (MQ) theories:

$$
\mathrm{MQ}^{(5)}[\mathbf{X}] \cong \mathrm{EQ}^{(4)}[\mathbf{X}] / \mathrm{U}(1)^{f}, \quad \quad \mathrm{MQ}^{(4)}[\mathbf{X}] \cong \mathrm{EQ}^{(5)}[\mathbf{X}] / \mathrm{U}(1)^{f}
$$

where the quotient denotes the $S$-type gauging of the $\mathrm{U}(1)^{f}$ flavor symmetry. We then propose that the electric and magnetic quiverines of $\mathcal{T}_{\mathbf{X}}^{5 \mathrm{~d}}$ and $\mathscr{T}_{\mathbf{X}}^{4 \mathrm{~d}}$, respectively, are $3 \mathrm{~d}$ 


\begin{tabular}{|c|c|c|c|}
\hline Singularity $\mathbf{X}$ & $\operatorname{SCFT} \mathcal{T}_{\mathbf{X}}^{5 \mathrm{~d}}$ & $\operatorname{SCFT} \mathscr{T}_{\mathbf{X}}^{4 \mathrm{~d}}$ & Dimension \\
\hline Divisor class number $\rho(\mathbf{X})$ & $\leq \operatorname{rank}\left(G_{H}^{5 \mathrm{~d}}\right)$ & $\leq \operatorname{rank}\left(G_{H}^{4 \mathrm{~d}}\right)$ & $f$ \\
Exceptional divisors & $\mathrm{CB}$ & reduced HB & $r$ \\
Kähler cone & extended CB & HB & $r+f=\widehat{d}_{H}$ \\
Strictly normalizable deformations & reduced HB & $\mathrm{CB}$ & $\widehat{r}$ \\
Normalizable deformations & HB & extended CB & $\widehat{r}+f=d_{H}$ \\
\hline
\end{tabular}

Table 1. 5d and 4d SCFTs from a canonical singularity X, at a glance. See section 2 for details.

mirror of each other ${ }^{3}$

$$
\mathrm{MQ}^{(5)} \quad 3 \mathrm{~d} \text { mirror } \quad \mathrm{EQ}^{(5)}, \quad \quad \mathrm{MQ}^{(4)} \stackrel{3 \mathrm{~d} \text { mirror }}{\Longleftrightarrow} \mathrm{EQ}^{(4)} .
$$

When it is an ordinary gauge-theory quiver, $\mathrm{MQ}^{(5)}$ coincides with the magnetic quiver for the $5 \mathrm{~d}$ SCFT $\mathcal{T}_{\mathbf{X}}^{5 \mathrm{~d}}$ as studied e.g. in [27, 31, 33, 76-78]. Similarly, the magnetic quiverine $\mathrm{MQ}^{(4)}$ of $\mathscr{T}_{\mathbf{X}}^{4 \mathrm{~d}}$ is usually known as the '3d mirror' of $\mathscr{T}_{\mathbf{X}}^{4 \mathrm{~d}}[43]$.

The most important aspect of $3 \mathrm{~d}$ mirror symmetry is that is exchanges the Higgs branch (HB) and the $3 \mathrm{~d}$ Coulomb branch (CB). Recall that, while the $5 \mathrm{~d}$ Coulomb branch of $\mathcal{T}_{\mathbf{X}}^{5 \mathrm{~d}}$ is a real cone and the $4 \mathrm{~d}$ Coulomb branch of $\mathscr{T}_{\mathbf{X}}^{4 \mathrm{~d}}$ is a complex (special Kähler) cone, the $\mathrm{CB}$ of a $3 \mathrm{~d} \mathcal{N}=4 \mathrm{SCFT}$ is itself a hyper-Kähler cone. On the other hand, the Higgs branch is not affected by toroidal compactification, and we then expect:

$$
\begin{aligned}
& \mathcal{M}_{H}\left[\mathcal{T}_{\mathrm{X}}^{5 \mathrm{~d}}\right] \cong \mathrm{HB}\left[\mathrm{EQ}^{(5)}\right] \cong \mathrm{CB}\left[\mathrm{MQ}^{(5)}\right] \\
& \mathcal{M}_{H}\left[\mathscr{T}_{\mathbf{X}}^{4 \mathrm{~d}}\right] \cong \mathrm{HB}\left[\mathrm{EQ}^{(4)}\right] \cong \mathrm{CB}\left[\mathrm{MQ}^{(4)}\right]
\end{aligned}
$$

Therefore, the quantum Higgs branches of $\mathcal{T}_{\mathbf{X}}^{5 \mathrm{~d}}$ and $\mathscr{T}_{\mathbf{X}}^{4 \mathrm{~d}}$ can be studied as quantum Coulomb branches of their magnetic quiverines. This may seem to exchange one hard problem with another, but it turns out that, by now, $3 \mathrm{~d} \mathcal{N}=4$ Coulomb branches are rather well understood (see e.g. [49-52]). This type of magnetic-quiver construction was first advocated in [76], and explored in numerous papers in recent years [27, 30-38].

We summarize some of the quantities of interest in table 1, which we will further explain in the next section. In particular, we denote by $r$ and $\widehat{r}$ the rank of $\mathcal{T}_{\mathbf{X}}^{5 \mathrm{~d}}$ and $\mathscr{T}_{\mathbf{X}}^{4 \mathrm{~d}}$, respectively, and by $d_{H}$ and $\widehat{d}_{H}$ the quaternionic dimension of their respective Higgs branches. We also have $f$ the rank of the $\mathrm{U}(1)^{f}$ flavor symmetry preserved on the $\mathrm{CB}$, which is generally a subgroup of the flavor symmetry $G_{H}^{5 \mathrm{~d}}$ or $G_{H}^{4 \mathrm{~d}}$ acting on the $5 \mathrm{~d}$ or $4 \mathrm{~d}$ SCFT Higgs branch. We have the key relations:

$$
d_{H}=\widehat{r}+f, \quad r+f=\widehat{d}_{H},
$$

whenever $\mathbf{X}$ is an isolated hypersurface singularity. (We will also briefly discuss the case of isolated toric singularities.)

Our analysis also clarifies subtle issues in the engineering of 5d SCFTs from isolated singularities. For instance, we will discuss the rank- $N E_{8} 5 \mathrm{~d}$ theory in detail, resolving

\footnotetext{
${ }^{3}$ Here and in the following, we often keep the dependence on the singularity $\mathbf{X}$ implicit, to avoid clutter.
} 
some interesting puzzle at $N \geq 2$. Another interesting piece of information that one can extract from the geometry is the set of higher-form symmetries of $\mathcal{T}_{\mathbf{X}}^{5 \mathrm{~d}}$ or $\mathscr{T}_{\mathbf{X}}^{4 \mathrm{~d}}$, following the approach of [79-81]. In particular, we will compute the one-form symmetry of many generalized Argyres-Douglas theories $\mathscr{T}_{\mathbf{X}}^{4 \mathrm{~d}}$ directly from the singularity data. ${ }^{4}$

\subsection{Comments on rank-0 theories from singularities}

The perspective we just sketched immediately leads to the following observations. First, the M-theory engineering predicts the existence of an infinite class of rank-zero theories in $5 \mathrm{~d}$ - which may or may not be interacting SCFTs. They correspond to terminal singularities - canonical singularities that do not admit crepant resolutions with exceptional divisors (so that $r=0$ ). For many of these $5 \mathrm{~d}$ rank-zero SCFTs $\mathcal{T}_{\mathbf{X}}^{5 \mathrm{~d}}$, the singularity $\mathbf{X}$ engineers a (generalized) Argyres-Douglas (AD) theory $\mathscr{T}_{\mathbf{X}}^{4 \mathrm{~d}}$ in IIB [42, 83-86]. Embedding the same terminal singularity in an elliptic Calabi-Yau threefold, one can also construct the $6 \mathrm{~d}$ uplift of these $5 \mathrm{~d}$ rank-zero SCFTs. Their contributions to the $6 \mathrm{~d}$ gravitational anomaly in a compact model was studied in $[87,88]$. However, it is unclear whether they give rise to non-trivial rank-0 theories in $6 \mathrm{~d}$ either.

Similarly, the IIB engineering predicts the existence of infinite families of rank-zero $4 \mathrm{~d}$ $\mathcal{N}=2$ theories, which may or may not be interacting, and correspond to singularities $\mathbf{X}$ whose complex-structure deformation $\widehat{\mathbf{X}}$ does not give rise to any dynamical $\mathrm{U}(1)$ vector multiplet in $4 \mathrm{~d}$ (so that $\widehat{r}=0$ ). This happens, in particular, for all isolated toric singularities; this point was first noted by [46] for toric orbifolds. The same singularities engineer well-studied (and conventional, higher rank) 5d SCFTs in M-theory.

To determine the properties of these rank- 0 theories in $5 \mathrm{~d}$ and $4 \mathrm{~d}$, in particular to clarify whether these should be viewed as genuine interacting SCFTs, or simply as discrete gaugings of free hypermultiplets, remains an interesting question. Discrete gauging in $4 \mathrm{~d}$ was studied e.g. in [89-92]; for discrete gauging in $3 \mathrm{~d}$ and 6d, see [93-95]. We will study these theories, mostly in $5 \mathrm{~d}$, by determining their magnetic quivers (or quiverines) according to (1.7), and thereby their Higgs branch. In all rank-zero cases we studied, we find that the $5 \mathrm{~d}$ Higgs branch is of the type $\mathbb{H}^{n} / \Gamma$, which hints at an interpretation in terms of discrete gauging. Either way, these theories provide an interesting new toolkit for constructions of 5d SCFTs in M-theory.

Another point of view is provided by the brane-webs and associated magnetic quivers and Hasse diagrams for the 5d Higgs branches. It was observed in [33, 36, 37] that some Hasse diagrams contain rank-0 slices, corresponding to e.g. $O(1)$ theories with $N$ flavors. Since such theories clearly appear as sub-structures in the Higgs branch of 5d SCFTs, an understanding of their geometric origin is very well motivated.

An additional tantalizing piece of evidence for the existence of these rank-zero theories in $5 \mathrm{~d}$ comes about by looking at higher-rank theories $\mathcal{T}_{\mathbf{X}}^{5 \mathrm{~d}}$. For instance, we find a large number of rank- 1 theories $\mathcal{T}_{\mathbf{X}}^{5 \mathrm{~d}}$, from canonical singularities with $r=1$, that can be viewed as an 'ordinary' rank-1 'coupled' to a rank-0 SCFT. These new theories differ in their Higgs

\footnotetext{
${ }^{4}$ These one-form symmetries were also studied in [82], which appeared on the arXiv at the same time as the first version of this paper.
} 
branches and in their global symmetries, in particular in their higher-form symmetries, from the 'underlying' rank-1 and rank-0 theories. We will give one specific example of this in this paper; many more examples, of essentially any rank, will be discussed in [10].

Our evidence in $4 \mathrm{~d}$ is somewhat more limited, but points towards the same conclusion, that these rank-0 $4 \mathrm{~d} \mathcal{N}=2$ theories, engineered at toric singularities [46], can be free hypermultiplets or discrete gauging thereof.

This paper is organised as follow. In section 2, we set the stage by reviewing some basic features of the geometric engineering of $5 \mathrm{~d}$ and $4 \mathrm{~d}$ SCFTs from canonical singularities, and we explore the relation between the two constructions via T-duality. In section 3, we explore this $5 \mathrm{~d} / 4 \mathrm{~d}$ correspondence in a few examples where both sides are well understood. In sections 4, we study the 5d theories that correspond to Argyres-Douglas in 4d, and are generally rank-0 $5 \mathrm{~d}$ theories. Finally, in section 5 , we discuss some $4 \mathrm{~d}$ theories engineered at toric singularities.

\section{$25 \mathrm{~d} / 4 \mathrm{~d}$ correspondence via $3 \mathrm{~d}$ mirror symmetry}

In this section, we first review some aspects of the geometric engineering of the $5 \mathrm{~d}$ SCFT $\mathcal{T}_{\mathbf{X}}^{5 \mathrm{~d}}$ and of the $4 \mathrm{~d}$ SCFT $\mathscr{T}_{\mathbf{X}}^{4 \mathrm{~d}}$ from an isolated canonical singularity $\mathbf{X}$. We then discuss how they are related by $3 \mathrm{~d}$ mirror symmetry. Finally, we shall briefly explain how to read off various higher-form symmetries from the geometry. The formalism reviewed here will be explained in more depth in [10].

\section{$2.15 d$ SCFTs from M-theory}

By now, there is a large amount of evidence for the proposition that the low-energy limit of M-theory on a singularity $\mathbf{X}$ defines for us a five-dimensional SCFT in the transverse directions, as in (1.2). While the $5 \mathrm{~d}$ superconformal fixed point is necessarily a strongly-coupled system [96, 97], it is an otherwise ordinary local unitary QFT. ${ }^{5}$ A standard approach, utilising its M-theory definition, is to deform and/or (crepantly) resolve the singularity $\mathbf{X}$ to obtain a smooth local (that is, non-compact) Calabi-Yau (CY) threefold. Then, standard methods (chiefly, the supergravity approximation and the study of BPS branes) become available. The parameter spaces of resolutions and of deformations are identified with the extended Coulomb branch and with the Higgs branch of the 5d SCFT, respectively

$\begin{array}{llll}\operatorname{ECB}\left(\mathcal{T}_{\mathbf{X}}^{5 \mathrm{~d}}\right) & \leftrightarrow & \text { resolutions (extended Kähler cone): } & \widetilde{\mathbf{X}} \\ \operatorname{HB}\left(\mathcal{T}_{\mathbf{X}}^{5 \mathrm{~d}}\right) & \leftrightarrow & \text { deformations }(\text { complex structure parameters): } & \widehat{\mathbf{X}}\end{array}$

The geometric approach to $\mathcal{T}_{\mathbf{X}}^{5 \mathrm{~d}}$ has mostly focussed on its Coulomb branch $(\mathrm{CB})$, whose low-energy dynamics is well-approximated by 11d supergravity [98]. Any smooth point

\footnotetext{
${ }^{5}$ One may think of this system, at least heuristically, as the result of decoupling $5 \mathrm{~d}$ gravity, in a compactification of M-theory on some Calabi-Yau threefold, by taking some large volume limit. Here, our point of view is to consider the canonical singularity $\mathbf{X}$ irrespective of any embedding in a compact $\mathrm{CY}_{3}$, which may or may not exist.
} 
of the (extended) $\mathrm{CB}$ is described by a complete crepant resolution $\pi: \widetilde{\mathbf{X}} \rightarrow \mathbf{X}$, which contains an exceptional locus:

$$
\pi^{-1}(0)=\bigcup_{a=1}^{r} S_{a}
$$

consisting of $r$ exceptional divisors intersecting along curves, assuming $r>0 .{ }^{6}$ The integer $r$, which is independent of the choice of crepant resolution, is called the rank of the 5d SCFT. The effective field theory on the CB consists of $r$ massless photons (and their superpartners in $5 \mathrm{~d} \mathcal{N}=1$ vector multiplets) with an action fully determined by the classical geometry $\widetilde{\mathbf{X}}[7]$. The low-energy BPS excitations are the M2-branes (electrically charged particles) and M5-branes (magnetically charged strings) wrapping 2- and 4-cycles, respectively, within the exceptional locus (2.2). We should also note that some of the Kähler parameters correspond to effective curves which are dual to non-compact divisors. These correspond to non-dynamical vector multiplets, or, equivalently, to mass terms for flavor symmetries. We denote by $f$ the rank of the flavor symmetry group from these curves, which is a subgroup of the actual flavor symmetry group, $G_{H}^{5 \mathrm{~d}}$, of $\mathcal{T}_{\mathbf{X}}^{5 \mathrm{~d}}$ (see section 2.4 for more details). The resolved singularity may also contain 3 -cycles, but on the other hand it is always simply connected $[99,100]$. We then have:

$$
\begin{aligned}
& \operatorname{dim} H_{1}(\tilde{\mathbf{X}}, \mathbb{R})=0, \quad \operatorname{dim} H_{3}(\tilde{\mathbf{X}}, \mathbb{R})=b_{3}, \\
& \operatorname{dim} H_{2}(\widetilde{\mathbf{X}}, \mathbb{R})=r+f, \quad \operatorname{dim} H_{4}(\widetilde{\mathbf{X}}, \mathbb{R})=r .
\end{aligned}
$$

The exceptional divisors and curves can be collapsed to zero volume by varying the Kähler parameters, thus recovering the UV SCFT $\mathcal{T}_{\mathbf{X}}^{5 \mathrm{~d}}$. At that point, the origin of the $5 \mathrm{~d} \mathrm{CB}$, mutually non-local particles and strings become massless, which is a strong indicator of the existence of a gapless phase [83]. The 3-cycles in the crepant resolution $\widetilde{\mathbf{X}}$ also provide free hypermultiplets on the five-dimensional CB. This can correspond to rather interesting physics, as we will see in some examples below, and in [10].

The SCFT $\mathcal{T}_{\mathbf{X}}^{5 \mathrm{~d}}$ may also have a Higgs branch $(\mathrm{HB})$, parameterized by the VEVs of scalar operators charged under the $\mathrm{SU}(2)_{R}$ superconformal R-symmetry. A generic point on the HB also spontaneously breaks the flavor symmetry group, $G_{H}^{5 \mathrm{~d}}$. The HB of any SQFT is a hyper-Kähler manifold. In any SCFT, it should also be a hyper-Kähler cone. We denote by $\mathcal{M}_{H}\left[\mathcal{T}_{\mathbf{X}}^{5 \mathrm{~d}}\right]$ (or $\mathcal{M}_{H}^{5 \mathrm{~d}}$ ) the HB of $\mathcal{T}_{\mathbf{X}}^{5 \mathrm{~d}}$, and by $d_{H}$ its quaternionic dimension

$$
\operatorname{dim} \mathcal{M}_{H}\left[\mathcal{T}_{\mathbf{X}}^{5 \mathrm{~d}}\right]=\frac{1}{2} \operatorname{dim}_{\mathbb{C}} \mathcal{M}_{H}\left[\mathcal{T}_{\mathbf{X}}^{5 \mathrm{~d}}\right]=d_{H}
$$

Upon deforming the singularity $\mathbf{X}$ to a smooth local CY threefold $\widehat{\mathbf{X}}$, we obtain a number of 3-cycles, $S_{l}^{3}, l=1, \cdots, \mu$, which are topologically three-spheres. The low-energy hypermultiplets consist of the complex-structure moduli:

$$
t_{l}=\int_{S_{l}^{3}} \Omega_{3}
$$

\footnotetext{
${ }^{6}$ The case $r=0$ instead corresponds to 'small resolutions', i.e. resolutions whose exceptional loci consist of curves only. In that case, we have real mass terms but no dynamical CB fields in $5 \mathrm{~d}$.
} 
very schematically, paired with the $5 \mathrm{~d}$ axions arising from the reduction of the M-theory 3 -form gauge field. Not all such hypermultiplets are dynamical, however [72]. The Higgs branch dimension $d_{H}$ is generally smaller than $\mu$. For $\mathbf{X}$ an IHS, one finds that:

$$
d_{H}=\widehat{r}+f,
$$

where $\widehat{r}$ is the dimension of the mixed Hodge structure (MHS) group $H^{1,2}(\widehat{\mathbf{X}})$ on the vanishing cohomology of $\mathbf{X}$. Indeed, for this class of singularities, we have [41, 43]:

$$
\mu=2 \widehat{r}+f, \quad f=\operatorname{dim} H^{2,2}(\widehat{\mathbf{X}}), \quad \widehat{r}=\operatorname{dim} H^{1,2}(\widehat{\mathbf{X}})=\operatorname{dim} H^{2,1}(\widehat{\mathbf{X}}) .
$$

The complex structure parameters in $H^{1,2}(\widehat{\mathbf{X}})$ corresponds to 'strictly normalizable' deformations ${ }^{7}$ of $\mathbf{X}$, while the 'normalizable deformations' also include the parameters from $H^{2,2}(\widehat{\mathbf{X}}) .{ }^{8}$ Importantly, the integer $f$ defined as in (2.7) coincide with the integer $f$ appearing in (2.3), as we explain further below.

Unlike the situation for the CB, 11d supergravity is not enough to compute the metric on the Higgs branch of $\mathcal{T}_{\mathbf{X}}^{5 \mathrm{~d}}$, due to the presence of M2-brane instantons wrapping the vanishing 3-cycles [101]. Instead, one must rely on other approaches, using various stringtheory dualities. A particularly fruitful approach, in recent years, has been the study of $(p, q)$-brane webs engineering $\mathcal{T}_{\mathbf{X}}^{5 \mathrm{~d}}[29]$, and the related concept of a "magnetic quiver" whose $3 \mathrm{~d}$ Coulomb branch is identical to the hyper-Kähler cone of the $\mathrm{HB}, \mathcal{M}_{H}\left[\mathcal{T}_{\mathbf{X}}^{5 \mathrm{~d}}\right]$, that we would like to understand $[27,30,31,33,34,38,102]$. One interesting outcome of the present work is that it provides a derivation from the geometry of magnetic quivers for the SCFT $\mathcal{T}_{\mathbf{X}}^{5 \mathrm{~d}}$ defined as in (1.2), at least in a number of cases [10]. We will see some examples of this in section 3 below.

\subsection{4d SCFTs from Type IIB}

The low-energy limit of Type IIB string theory on a canonical singularity $\mathbf{X}$ defines a $4 \mathrm{~d}$ $\mathcal{N}=2$ SCFT $\mathscr{T}_{\mathbf{X}}^{4 \mathrm{~d}},(1.3)$, in the transverse directions [8, 42-45, 103-105]. In this context, the complex structure deformations correspond to the (extended) Coulomb branch of the SCFT, while the Kähler cone of $\mathbf{X}$ underlies the Higgs branch of the theory:

$$
\begin{array}{llll}
\operatorname{ECB}\left(\mathscr{T}_{\mathbf{X}}^{4 \mathrm{~d}}\right) & \leftrightarrow & \text { deformations }(\text { complex structure parameters): } & \widehat{\mathbf{X}} \\
\operatorname{HB}\left(\mathscr{T}_{\mathbf{X}}^{4 \mathrm{~d}}\right) & \leftrightarrow & \text { resolutions (Kähler parameters): } & \widetilde{\mathbf{X}}
\end{array}
$$

Here, the beautiful structure of the $4 \mathrm{~d} \mathcal{N}=2$ Coulomb branch, which must be a special Kähler cone, is encoded in the classical geometry of the deformed singularity $\mathbf{X}$. By contrast, the 4d Higgs branch metric receives quantum corrections from D1- and D3-brane instantons wrapping the exceptional locus of $\widetilde{\mathbf{X}}$.

\footnotetext{
${ }^{7}$ By which we mean, such that $\Delta>1$, with the scaling dimension $\Delta$ defined in (2.13) below; normalizable deformations have $\Delta \geq 1$.

${ }^{8}$ It may be worth pointing out that the $H^{2,2}$ MHS group does not correspond to 4-forms (it is not a Dolbeault cohomology group). For a pedagogical review of MHS in our context, we refer to [10].
} 
For concreteness, let us focus on the case of quasi-homogeneous isolated hypersurface singularities (1.1), as first studied in [8]. The $4 \mathrm{~d}$ SCFT $\mathscr{T}_{\mathbf{X}}^{4 \mathrm{~d}}$ must have a superconformal $R$ symmetry $\mathrm{U}(1)_{r}$ which is spontaneously broken on the Coulomb branch. This corresponds to the condition that the defining polynomial $F(x)$ be quasi-homogeneous

$$
F\left(\lambda^{q_{1}} x_{1}, \lambda^{q_{2}} x_{2}, \lambda^{q_{3}} x_{3}, \lambda^{q_{4}} x_{4}\right)=\lambda F\left(x_{1}, x_{2}, x_{3}, x_{4}\right),
$$

for some scaling weights $q_{i} \in \mathbb{Q}_{>0}$. The requirement that the singularity be canonical translates to the condition $\sum_{i=1}^{4} q_{i}>1$. The deformed singularity, corresponding to the $\mathrm{CB}$, takes the form:

$$
\widehat{\mathbf{X}} \cong\left\{\widehat{F}(x) \equiv F(x)+\sum_{l=1}^{\mu} t_{l} x^{\mathfrak{m}_{l}}=0\right\},
$$

where $x^{\mathfrak{m}_{l}}$ denote the monomials generating the Milnor ring

$$
\mathcal{M}(F)=\mathbb{C}\left[x_{1}, x_{2}, x_{3}, x_{4}\right] /(d F),
$$

and $\mu$ is the Milnor number of the singularity, where one has the relation

$$
\mu=\prod_{i=1}^{4}\left(q_{i}^{-1}-1\right) .
$$

At generic values of the complex-structure parameters $t_{l}$, the deformed singularity is smooth and has the homotopy type of a 'bouquet' of $\mu$ three-spheres. Let us order the deformations $t_{l}$ according to their weights $Q[t]$ under (2.9). They have conformal dimensions

$$
\Delta\left[t_{l}\right]=\frac{Q\left[t_{l}\right]}{\sum_{i=1}^{4} q_{i}-1} .
$$

One then identifies the deformation parameters with dimensions $\Delta>1$ as the $\mathrm{CB}$ ' $u$ parameters' - the VEVs $u_{l} \equiv\left\langle\mathcal{O}_{l}\right\rangle$ of the CB operators $\mathcal{O}_{l}$ - while the parameters with $\Delta<1$ are supersymmetric deformations of the SCFT (that is, deformations of the $4 \mathrm{~d}$ theory by the F-terms $\left.\int d^{4} \theta \mathcal{O}_{l}\right)$. We denote by $\widehat{r}$ the number of $\mathrm{CB}$ operators - that is, the rank of the SCFT, whose CB is freely generated. Each independent CB operator is paired with a corresponding F-term deformation, with

$$
\Delta\left[t_{l}\right]+\Delta\left[t_{\mu-l+1}\right]=2, \quad l=1, \cdots \widehat{r} .
$$

The deformations with $\Delta=1$, on the other hand, are unpaired, and correspond to complex mass terms, which are VEVs for background vector multiplets for the maximal torus of the flavor symmetry group, $G_{H}^{4 \mathrm{~d}}$, of $\mathscr{T}_{\mathbf{X}}^{4 \mathrm{~d}}$. Let us also mention that, by using the ShapereTachikawa relations [106], the conformal central charges $a$ and $c$ can be easily computed from the spectrum (2.13) [43]. See also [54] for some more recent developments.

The Seiberg-Witten geometry [107] of $\mathscr{T}_{\mathbf{X}}^{4 \mathrm{~d}}$ is entirely captured by the local Calabi-Yau $\widehat{\mathbf{X}}$ fibered over the extended Coulomb branch (ECB), corresponding to the 'normalizable parameters' $t_{l}$ such that $\Delta\left[t_{l}\right] \geq 1$. The low-energy physics is therefore determined by an $\mathrm{SL}(\mu, \mathbb{Z})$ bundle over the ECB, with the SW periods computed classically as

$$
a_{l}=\int_{A_{l}} \Omega_{3}, \quad a_{D, l}=\int_{B_{l}} \Omega_{3}, \quad l=1, \cdots, \widehat{r}, \quad m_{\alpha}=\int_{L} \Omega_{3}, \quad \alpha=1, \cdots, f,
$$


in some appropriate basis of $H_{3}(\widehat{\mathbf{X}}, \mathbb{Z})$; the holomorphic 3 -form $\Omega_{3}$ of the non-compact CY threefold $\widehat{\mathbf{X}}$ generalizes the SW differential $\lambda_{\mathrm{SW}}$ [103]. The non-trivial structure of the SW fibration can be studied using Picard-Lefschetz theory. In particular, one can straightforwardly compute the monodromy group of the $4 \mathrm{~d}$ ECB from the singularity data. Let us also recall that the Coulomb-branch BPS particles are realized as D3-branes wrapped over the vanishing 3 -cycles [8], and that the Milnor number $\mu=2 \widehat{r}+f$ is the dimension of the electro-magnetic charge lattice, extended with the flavor charges.

\subsection{Crepant resolution and anomaly matching on the Higgs branch of $\mathscr{T}_{\mathrm{X}}^{4 \mathrm{~d}}$}

The Higgs branch of $\mathscr{T}_{\mathbf{X}}^{4 \mathrm{~d}}$ is engineered in IIB as a torus fibration over the complexified Kähler cone of the singularity — see e.g. [108-110]. While the HB metric is strongly affected by the D-brane instantons, we can nonetheless understand the basic features of the Higgs phase by studying the topology of a generic crepant resolution, $\widetilde{\mathbf{X}}$. The number of hypermultiplets, $\widehat{d}_{H}$, at a generic point on the HB, is given by the number of 2-cycles (2.3), $\widehat{d}_{H}=r+f-$ that is, the number of Kähler parameters. The number of massless vector multiplets at such a Higgs-branch point, on the other hand, is given by $\frac{1}{2} b_{3}$, half the number of 3-cycles in the crepant resolution (they come in pairs [99]). Finally, there might remain some terminal singularities $\mathbf{X}_{\mathrm{IR}}$ on the exceptional locus of the resolution $\widetilde{\mathbf{X}}$, which cannot be resolved further. Such singularities are interpreted as 'irreducible' SCFTs, which live at every point on the Higgs branch. (This general structure of the Higgs branch was discussed from the VOA perspective in [70].) To summarize, we have the following Higgs-branch lowenergy effective theory:

$$
\mathcal{T}_{\mathrm{HB}}\left[\mathscr{T}_{\mathbf{X}}^{4 \mathrm{~d}}\right] \cong\left(\widehat{d}_{H} \text { hypers }\right) \oplus\left(\frac{1}{2} b_{3} \text { vectors }\right) \oplus \mathscr{T}_{\mathbf{X}_{\mathrm{IR}}}^{4 \mathrm{~d}} \quad \leftrightarrow \quad(2 \text {-cycles }) \oplus(3 \text {-cycles }) \oplus \mathbf{X}_{\mathrm{IR}}
$$

with the indicated geometric correspondence. The IR SCFT is a tensor product of distinct SCFTs whenever there are several distinct residual terminal singularities. We can provide a strong check of this picture by matching the 't Hooft anomaly $\operatorname{Tr}\left(\mathrm{U}(1)_{r}\right)$, since $\mathrm{U}(1)_{r}$ is preserved on the Higgs branch. For the SCFT in the UV, the anomaly is simply related to the conformal anomalies [106]:

$$
\mathcal{A}_{r}\left[\mathscr{T}_{\mathbf{X}}^{4 \mathrm{~d}}\right]=24(c-a)=n_{h}-n_{v},
$$

where $n_{h}=-16 a+20 c$ and $n_{v}=8 a-4 c$ are the 'effective' number of hypermultiplet and vector multiplets, respectively, at the fixed point. Upon going on the Higgs branch, we have to match with the anomaly of the IR theory (2.16):

$$
\mathcal{A}_{r}\left[\mathcal{T}_{\mathrm{HB}}\right]=\widehat{d}_{H}-\frac{1}{2} b_{3}+\mathcal{A}_{r}\left[\mathscr{T}_{\mathbf{X}_{\mathrm{IR}}^{4 \mathrm{~d}}}\right]
$$

Thus, we must have:

$$
24(c-a)=\widehat{d}_{H}-\frac{1}{2} b_{3}+24\left(c^{\mathrm{IR}}-a^{\mathrm{IR}}\right),
$$

where $a^{\mathrm{IR}}, c^{\mathrm{IR}}$ are the central charges of the IR SCFT $\mathscr{T}_{\mathbf{X}_{\mathrm{IR}}}^{4 \mathrm{~d}}$. (See $[111,112]$ for related discussions.) This anomaly matching condition provides a strong check on the geometric 
engineering picture, since it looks quite miraculous in terms of the geometry, relating the deformation data (which encodes $a-c$ ) to the crepant resolution data. We checked this relation explicitly in many examples. It would be very interesting to prove it directly from the geometry. For future reference, let us also define the quantity

$$
\Delta \mathcal{A}_{r} \equiv 24(c-a)-\widehat{d}_{H}=-\frac{1}{2} b_{3}+24\left(c^{\mathrm{IR}}-a^{\mathrm{IR}}\right),
$$

which vanishes if the Higgs branch consists of free hypermultiplets only. ${ }^{9}$

\subsection{Flavor symmetries, conifold transitions and boundary five-manifold}

For $\mathbf{X}$ a hypersurface singularity, we have seen that the integer denoted by $f$ appears in two distinct ways:

$$
\begin{aligned}
f & =\rho(\mathbf{X}) \\
& =\operatorname{dim} H^{2,2}(\widehat{\mathbf{X}}) .
\end{aligned}
$$

On the first line, $\rho(\mathbf{X})$ is the divisor class number - that is, the number of non-compact divisors, or, equivalently, the number of compact 2 -cycles $\mathcal{C}_{\alpha}, \alpha=1, \cdots, f$, to which they are dual, in any crepant resolution $\widetilde{\mathbf{X}}$. They give rise to background vector multiplets in the Coulomb phase of the $5 \mathrm{~d}$ SCFT $\mathcal{T}_{\mathbf{X}}^{5 \mathrm{~d}}$, in the M-theory construction. Since every conserved current in $\mathcal{T}_{\mathbf{X}}^{5 \mathrm{~d}}$ gives rise to an $\mathrm{SU}(2)_{R}$-preserving massive deformation of the $5 \mathrm{~d}$ fixed point [96], we would naively think that all such mass terms should be visible as Kähler parameters of the resolved singularity. This is however not the case, which is related to the possible presence of $b_{3} \in 2 \mathbb{Z} 3$-cycles in the resolved geometry. We claim that the flavor rank of $\mathcal{T}_{\mathbf{X}}^{5 \mathrm{~d}}$ is given by:

$$
\operatorname{rank}\left(G_{H}^{5 \mathrm{~d}}\right)=f+n_{\mathrm{ssb}}, \quad n_{\mathrm{ssb}} \leq \frac{1}{2} b_{3} .
$$

Here, the interpretation of the 3 -cycles in $\tilde{\mathbf{X}}$ is that some operators in $\mathcal{T}_{\mathbf{X}}^{5 \mathrm{~d}}$ have been given a VEV, landing us in a 'partial Higgs phase,' which spontaneously breaks a subgroup $\mathrm{U}(1)^{n_{\text {ssb }}} \subset G_{H}^{5 \mathrm{~d}}$ of the flavor symmetry of $\mathcal{T}_{\mathbf{X}}^{5 \mathrm{~d}}$. The number $n_{\text {ssb }}$ must be computed in a case-by-case basis, as it depends on the details of the resolution (we will see examples of this in the next section) - it corresponds to the number of Kähler parameters that can be gained by geometric transitions on these 3 -cycles, which preserve the $5 \mathrm{~d}$ Coulomb phase.

On the second line of (2.21), $f$ is defined as the number of 'unpaired' three-cycles in any generic deformation of the singularity. These are the compact 3 -cycles $S_{\alpha}^{3} \subset \widehat{\mathbf{X}}$, $\alpha=1, \cdots, f$, dual to non-compact 3 -cycles, which give rise to background vector multiplets in the Coulomb phase of the $4 \mathrm{~d}$ SCFT $\mathscr{T}_{\mathbf{X}}^{4 \mathrm{~d}}$, in the IIB construction, and $f$ is then identified as the rank of the flavor symmetry of $\mathscr{T}_{\mathbf{X}}^{4 \mathrm{~d}}$. Since there cannot be any 2- and 4-cycles in the deformation $\widehat{\mathbf{X}}$ of an IHS [113], we do not have any mixed Higgs phase on the CB of $\mathcal{T}_{\mathbf{X}}^{5 \mathrm{~d}}$, and therefore we expect that

$$
\operatorname{rank}\left(G_{H}^{4 \mathrm{~d}}\right)=f .
$$

\footnotetext{
${ }^{9}$ The converse is not true, of course, since we can have the coincidence that $\Delta \mathcal{A}_{r}=0$ because $b_{3}=$ $48\left(c^{\mathrm{IR}}-a^{\mathrm{IR}}\right)$. On the other hand, $\Delta \mathcal{A}_{r} \neq 0$ implies that the HB theory includes additional degrees of freedom besides the hypermultiplets, and $\Delta \mathcal{A}_{r}$ non-integer implies that there is some irreducible IR SCFT.
} 
Note that, even when $b_{3}=0$ and $G_{H}^{5 \mathrm{~d}}$ and $G_{H}^{4 \mathrm{~d}}$ have the same rank, the flavor groups $G_{H}^{5 \mathrm{~d}}$ and $G_{H}^{4 \mathrm{~d}}$ themselves, which act as $\mathrm{SU}(2)_{R}$-preserving isometries on the quantum Higgs branches $\mathcal{M}_{H}\left[\mathcal{T}_{\mathbf{X}}^{5 \mathrm{~d}}\right]$ and $\mathcal{M}_{H}\left[\mathscr{T}_{\mathbf{X}}^{4 \mathrm{~d}}\right]$, will in general not be the same.

Geometrically, there are two related ways to understand the 'coincidence' (2.21). The first one is that there exists a geometric transition that shrinks the curve $\mathcal{C}_{\alpha} \subset \widetilde{\mathbf{X}}$ to zero size, before turning on the complex structure deformation that gives rise to the 3-cycle $S_{\alpha}^{3}$, or vice versa, generalizing the well-studied conifold transition $[9,114]$ :

$$
\mathcal{C}_{\alpha} \quad \longleftrightarrow \quad S_{\alpha}^{3}
$$

Such a transition, in general, has to go through the singularity $\mathbf{X}$ at the common origin the Kähler and complex structure parameter spaces. Physically, in either the $5 \mathrm{~d}$ or $4 \mathrm{~d}$ interpretation, this transition $\left(\mathcal{C}_{\alpha} \rightarrow S_{\alpha}^{3}\right.$ in M-theory, or $S_{\alpha}^{3} \rightarrow \mathcal{C}_{\alpha}$ in IIB) corresponds to sending a mass to zero, upon which some field charged under the flavor symmetry $\mathrm{U}(1)_{\alpha}$ becomes massless, and can be then given a VEV that spontaneously breaks the symmetry.

The second, related explanation is that both the 2-cycles $\mathcal{C}_{\alpha}$ and the 3 -cycles $S_{\alpha}^{3}$ sit in the image of the embedding map for the boundary into the threefold, namely

$$
\mathcal{C}_{\alpha} \in \operatorname{im}\left[H_{2}(\partial \widetilde{\mathbf{X}}, \mathbb{Z}) \rightarrow H_{2}(\widetilde{\mathbf{X}}, \mathbb{Z})\right], \quad S_{\alpha}^{3} \in \operatorname{im}\left[H_{3}(\partial \widehat{\mathbf{X}}, \mathbb{Z}) \rightarrow H_{3}(\widehat{\mathbf{X}}, \mathbb{Z})\right] .
$$

For an isolated singularity, the act of resolving or deforming the singularity does not affect the boundary at infinity, and we have

$$
\partial \widetilde{\mathbf{X}} \cong \partial \widehat{\mathbf{X}} \cong L_{5}(\mathbf{X})
$$

Here, $L_{5}(\mathbf{X})$ is the link of the singularity $\mathbf{X} .{ }^{10}$ For quasi-homogeneous isolated hypersurface singularities, $L_{5}(\mathbf{X})$ is simply-connected [113], so that its homology takes the general form:

$$
\begin{array}{lll}
H_{0}\left(L_{5}, \mathbb{Z}\right)=\mathbb{Z}, & H_{1}\left(L_{5}, \mathbb{Z}\right)=0, & H_{2}\left(L_{5}, \mathbb{Z}\right)=\mathbb{Z}^{f} \oplus \mathfrak{h}_{2}, \\
H_{3}\left(L_{5}, \mathbb{Z}\right)=\mathbb{Z}^{f}, & H_{4}\left(L_{5}, \mathbb{Z}\right)=0, & H_{5}\left(L_{5}, \mathbb{Z}\right)=\mathbb{Z},
\end{array}
$$

Both $f$ and the finite abelian group $\mathfrak{h}_{2}$ can be computed directly from the weights $q_{i}[116$, 117]. (See [10] for a review of the relevant combinatorial formulas.) We will discuss the physical meaning of Tor $H_{2}\left(L_{5}, \mathbb{Z}\right)=\mathfrak{h}_{2}$ in subsection 2.7.

\subsection{Circle reductions and the electric quiverines}

In both the M-theory engineering of $\mathcal{T}_{\mathbf{X}}^{5 \mathrm{~d}}$ and the Type IIB engineering of $\mathscr{T}_{\mathbf{X}}^{4 \mathrm{~d}}$, the extended Coulomb branch geometry arises semi-classically in string theory. On the one hand, the ECB of $\mathcal{T}_{\mathbf{X}}^{5 \mathrm{~d}}$ is identified with the extended Kähler cone of the singularity, of real dimension $r+f$, parameterizing the crepant resolutions of $\mathbf{X}$ (see e.g. [15] for a more detailed review). On the other hand, the ECB of $\mathscr{T}_{\mathbf{X}}^{4 \mathrm{~d}}$ is identified with the versal family of complex structure

\footnotetext{
${ }^{10}$ If the singularity admits a Ricci-flat metric, the five-dimensional link admits a Sasaki-Einstein metric (by definition). Here, we will not assume this, as there are generally obstructions to the existence of a SE metric — see [115] for a nice review. The non-existence of $\mathrm{SE}_{5}$ metrics, for many singularities of physical interest for geometric engineering, raises quite interesting questions, which however go beyond the scope of this paper.
} 
deformations, with the special-Kähler conical structure arising naturally from the vanishing 3-cycles.

By contrast, the Higgs branch in either case is much harder to study, since it receives M2- or D-brane instanton corrections. From the assumed superconformal invariance, we know that it must be a hyper-Kähler cone, and, from the geometric engineering, we know its quaternionic dimension:

$$
\operatorname{dim} \mathcal{M}_{H}\left[\mathcal{T}_{\mathbf{X}}^{5 \mathrm{~d}}\right]=d_{H}=\widehat{r}+f, \quad \operatorname{dim} \mathcal{M}_{H}\left[\mathscr{T}_{\mathbf{X}}^{4 \mathrm{~d}}\right]=\widehat{d}_{H}=r+f,
$$

in $5 \mathrm{~d}$ and $4 \mathrm{~d}$, respectively. Note that, in this paper, we are always talking about the 'quantum Higgs branch' of the SCFT [57], which generally does not have any Lagrangian description - in particular, the Higgs branch may not be realizable as a hyper-Kähler quotient. Of course, one could also consider mixed branches, which correspond to partially resolving then deforming the singularity, or vice versa.

It is clear, from the discussion so far, that the Higgs branch data and the Coulomb branch data of the $5 \mathrm{~d}$ and $4 \mathrm{~d}$ SCFTs are related to each other. In particular, we see from (2.28) that the quaternionic Higgs branch dimension in $5 \mathrm{~d}, d_{H}$, is equal to the complex dimension of the extended Coulomb branch of $\mathscr{T}_{\mathbf{X}}^{4 \mathrm{~d}}$. In this subsection and the next, we spell out this relation in more detail.

Let us first consider compactifying the theory $\mathscr{T}_{\mathbf{X}}^{4 \mathrm{~d}}$ on a finite-size circle. This gives rise to a three-dimensional $\mathcal{N}=4$ supersymmetric field theory, whose Coulomb branch is now hyper-Kähler - each $4 \mathrm{~d} \mathcal{N}=2$ abelian vector multiplet gives us a $3 \mathrm{~d} \mathcal{N}=4$ vector multiplet, which contains three real scalars plus a (dual) photon, and the hyper-Kähler structure is dictated by $3 \mathrm{~d} \mathcal{N}=4$ supersymmetry. Note that this happens as soon as we compactify the theory on $S^{1}$, at any radius $[118,119]$. The $3 \mathrm{~d} \mathcal{N}=4 \mathrm{CB}$ receives quantum corrections which dramatically correct its metric — say, compared to a semi-classical description for a $4 \mathrm{~d}$ Lagrangian theory on $\mathbb{R}^{3} \times S^{1}$. In string theory, these corrections arise from D3-branes instantons wrapping the circle times any vanishing 3-cycle in $\widehat{\mathbf{X}}$. Let $D_{S^{1}} \mathscr{T}_{\mathbf{X}}^{4 \mathrm{~d}}$ denote the KK $3 \mathrm{~d} \mathcal{N}=4$ theory obtained by circle compactification. In the far IR (compared to the KK scale), we obtain a new $3 \mathrm{~d} \mathcal{N}=4 \mathrm{SCFT}$, which we denote by:

$$
\mathrm{EQ}^{(4)}[\mathbf{X}] \stackrel{3 \mathrm{~d}}{\cong} D_{S^{1}} \mathscr{T}_{\mathbf{X}}^{4 \mathrm{~d}} .
$$

More precisely, we should think of $\mathrm{EQ}^{(4)}[\mathbf{X}]$ (also written as $\mathrm{EQ}^{(4)}$, with the dependence on the choice of singularity $\mathbf{X}$ implicit) as a three-dimensional SQFT which coincides with $D_{S^{1}} \mathscr{T}_{\mathbf{X}}^{4 \mathrm{~d}}$ below the KK scale - for $\mathscr{T}_{\mathbf{X}}^{4 \mathrm{~d}}$ given by a superconformal Lagrangian, it would simply be the dimensionally-reduced Lagrangian theory; for non-Lagrangian theories (the generic case), we need to work harder. The $3 \mathrm{~d} \mathcal{N}=4 \mathrm{SQFT} \mathrm{EQ}^{(4)}$ is called the 'electric quiver(ine)' of the $4 \mathrm{~d}$ SCFT $\mathscr{T}_{\mathbf{X}}^{4 \mathrm{~d}}$. Its IR fixed point is the $3 \mathrm{~d} \mathcal{N}=4$ SCFT we are interested in. It has a scale-invariant Coulomb branch, which must be a hyper-Kähler cone of quaternionic dimension

$$
\operatorname{dim} \mathrm{CB}\left[\mathrm{EQ}^{(4)}[\mathbf{X}]\right]=\widehat{r},
$$

while its Higgs branch, of dimension $\widehat{d}_{H}$, coincides with the Higgs branch of $\mathscr{T}_{\mathbf{X}}^{4 \mathrm{~d}}$. Note that the $\mathrm{SU}(2)_{C} R$-symmetry acting on $\mathrm{CB}\left[\mathrm{EQ}^{(4)}\right]$ arises as an accidental IR symmetry, af- 
ter non-trivial mixing between the $\mathrm{U}(1)_{r}$ symmetry of $\mathscr{T}_{\mathbf{X}}^{4 \mathrm{~d}}$ and $3 \mathrm{~d}$ topological symmetries that are present along the RG flow [120].

Similarly, we may consider the $5 \mathrm{~d}$ SCFT $\mathcal{T}_{\mathbf{X}}^{5 \mathrm{~d}}$ on a finite-size torus, which defines for us a KK field theory denoted by $D_{T^{2}} \mathcal{T}_{\mathbf{X}}^{5 \mathrm{~d}}$. This gives us another $3 \mathrm{~d} \mathcal{N}=4$ SCFT in the deep IR, and we denote the intermediate $3 \mathrm{~d} \mathcal{N}=4$ SQFT by

$$
\mathrm{EQ}^{(5)}[\mathbf{X}] \stackrel{3 \mathrm{~d}}{\cong} D_{T^{2}} \mathcal{T}_{\mathbf{X}}^{5 \mathrm{~d}}
$$

Since $\mathcal{T}_{\mathbf{X}}^{5 \mathrm{~d}}$ is never given by a superconformal Lagrangian, the 'electric quiver' $\mathrm{EQ}^{(5)}$ is not something we can read off easily. (Note, in particular, that this is not the dimensional reduction of some 5d IR gauge-theory description which may arise on the ECB of the $5 \mathrm{~d}$ SCFT.) By definition, the electric quiver of $\mathcal{T}_{\mathbf{X}}^{5 \mathrm{~d}}$ has a Coulomb branch of dimension

$$
\operatorname{dim} \mathrm{CB}\left[\mathrm{EQ}^{(5)}[\mathbf{X}]\right]=r
$$

while its Higgs branch, of dimension $d_{H}$, coincides with the Higgs branch of $\mathcal{T}_{\mathbf{X}}^{5 \mathrm{~d}}$.

As anticipated in the introduction, the $\mathcal{T}_{\mathbf{X}}^{5 \mathrm{~d}}$ on a torus and $\mathscr{T}_{\mathbf{X}}^{4 \mathrm{~d}}$ are related by T-duality in Type II string theory, as suggested by our construction

$$
D_{T_{2}} \mathcal{T}_{\mathbf{X}}^{5 \mathrm{~d}} \leftrightarrow \quad \leftrightarrow \quad \text { IIA on } \mathbb{R}^{3} \times S_{\beta}^{1} \times \mathbf{X} \quad \leftrightarrow \quad \text { IIB on } \mathbb{R}^{3} \times S_{\frac{1}{\beta}}^{1} \times \mathbf{X} \quad \leftrightarrow \quad D_{S^{1}} \mathscr{T}_{\mathbf{X}}^{4 \mathrm{~d}},
$$

and it is known that T-duality realizes $3 \mathrm{~d} \mathcal{N}=4$ mirror symmetry as a perturbative stringtheory symmetry [9] (we can keep $g_{s}$ very small in this whole discussion, by taking the Mtheory circle to be small). Thus, we expect the two $3 \mathrm{~d} \mathcal{N}=4$ effective descriptions (2.29) and (2.31) to be related by mirror symmetry. This, however, cannot be exactly true in our setup, since the dimension of the $\mathrm{CB}$ of $\mathrm{EQ}^{(4)},(2.30)$, does not match the dimension of the $\mathrm{HB}$ of $\mathrm{EQ}^{(5)}\left(d_{H}=\widehat{r}+f\right)$ unless $f=0$, and vice versa. The discrepancy comes from the $f \mathrm{U}(1)$ vectors in either description (4d in IIB, or $5 \mathrm{~d}$ in M-theory), which we discussed in subsection 2.4 .

In the following, we give a natural prescription to explicitly relate the SQFT descriptions of the $4 \mathrm{~d}$ and $5 \mathrm{~d}$ SCFTs compactified to 3d. A more detailed explanation of our approach will be given in [10].

\subsection{Higgs branches, magnetic quiverines and 3d mirrors}

Let us first define the magnetic quiverine of the $4 \mathrm{~d}$ SCFT $\mathscr{T}_{\mathbf{X}}^{4 \mathrm{~d}}$ as the $3 \mathrm{~d} \mathcal{N}=4$ mirror dual to its electric quiver: ${ }^{11}$

$$
\mathrm{MQ}^{(4)} \quad \stackrel{3 \mathrm{~d} \text { mirror }}{\Longleftrightarrow} \quad \mathrm{EQ}^{(4)}
$$

This magnetic quiverine - by the definition, the $3 \mathrm{~d}$ mirror description of the $3 \mathrm{~d}$ SCFT obtained by compactifying the $4 \mathrm{~d}$ SCFT on a circle - is more often called the $3 d$ mirror of $\mathscr{T}_{\mathbf{X}}^{4 \mathrm{~d}}$. In recent years, $3 \mathrm{~d}$ mirror theories have been obtained for many strongly-coupled theories, in particular for many Argyres-Douglas theories [86, 121-123]. The interesting point is that $\mathrm{MQ}^{(4)}$ may have an explicit Lagrangian description in $3 \mathrm{~d}$, even if $\mathrm{EQ}^{(4)}$ does not.

\footnotetext{
${ }^{11}$ Recall that we use the term 'quiverine' for the $3 \mathrm{~d}$ theories in general, which may not have a description as a standard quiver gauge theory.
} 
Similarly, we define the magnetic quiverine of the $5 \mathrm{~d} \operatorname{SCFT} \mathcal{T}_{\mathbf{X}}^{5 \mathrm{~d}}$ as the $3 \mathrm{~d}$ mirror

$$
\mathrm{MQ}^{(5)} \quad 3 \mathrm{~d} \text { mirror } \quad \mathrm{EQ}^{(5)}
$$

In the recent literature, the magnetic quiver (MQ) of any SQFT with eight Poincaré supercharges, $\mathcal{T}$, has been defined as a useful 'auxiliary' construction (generally constructed from a brane-web diagram), from which the Higgs branch of $\mathcal{T}$ can be computed as a $3 \mathrm{~d}$ $\mathcal{N}=4$ Coulomb branch

$$
\mathcal{M}_{H}[\mathcal{T}]=\mathrm{CB}[\mathrm{MQ}]
$$

The geometric-engineering approach gives us a fully geometric realization of this perspective [31], at least abstractly. Indeed, we really ought to define the magnetic quiver(ine)s directly from the string 'compactification', as follows.

Let us first consider the $5 \mathrm{~d} \operatorname{SCFT} \mathcal{T}_{\mathbf{X}}^{5 \mathrm{~d}}$ on $T^{2} \cong S_{M}^{1} \times S_{\beta}^{1}$, as in (2.31). It is well appreciated that abelian flavor symmetries in $3 \mathrm{~d}$ can be gauged and 'ungauged' at minimal cost $[74,75]$. The theory $\mathrm{EQ}^{(5)}$ has a $\mathrm{U}(1)^{f}$ flavor symmetry, ${ }^{12}$ which acts on its Higgs branch as an isometry commuting with the $\mathrm{SU}(2)_{R}$ action. We may then gauge this symmetry, to obtain a new theory, denoted by:

$$
\mathrm{MQ}^{(4)} \equiv \mathrm{EQ}^{(5)} / \mathrm{U}(1)^{f} .
$$

This gauging operation takes a theory that depends on background multiplets $V_{(F)}$ for $\mathrm{U}(1)^{f}$ and gives us another theory with a new topological symmetry $\mathrm{U}(1)_{T}^{f}$ (one topological current for each gauged $\mathrm{U}(1)$ ) coupled to background vector multiplets $V_{(T)}$. Schematically, we have:

$$
S_{\mathrm{MQ}^{(4)}}\left[V_{(T)}\right]=-\log \int\left[D V_{(F)}\right] \exp \left(-S_{\mathrm{MQ}^{(5)}}\left[V_{(F)}\right]-\frac{i}{2 \pi} \int A_{(T)} \wedge d A_{(F)}+\cdots\right),
$$

where $A$ denotes the gauge fields in vector multiplets, and the ellipsis denotes the supersymmetric completion (including Fayet-Iliopoulos terms). In particular, this so-called ' $S$ operation' sends an SCFT to another SCFT [75]. The operation is reversible; if we now gauge the new topological symmetries of $\mathrm{MQ}^{(4)}$, we obtain the original theory: ${ }^{13}$

$$
\mathrm{EQ}^{(5)} \equiv \mathrm{MQ}^{(4)} / \mathrm{U}(1)_{T}^{f}
$$

Note also that the gauging operation (2.37) acts on the Higgs branch of the theory as a hyper-Kähler quotient ${ }^{14}$

$$
\mathrm{HB}\left[\mathrm{MQ}^{(4)}\right]=\mathcal{M}_{H}\left[\mathcal{T}_{\mathbf{X}}^{5 \mathrm{~d}}\right] / / / \mathrm{U}(1)^{f},
$$

while it increases the dimension of the Coulomb branch by $f$ (roughly speaking, the ECB becomes the $\mathrm{CB}$ ). In particular, we have:

$$
\operatorname{dim} \mathrm{HB}\left[\mathrm{MQ}^{(4)}\right]=d_{H}-f=\widehat{r}, \quad \operatorname{dim} \mathrm{CB}\left[\mathrm{MQ}^{(4)}\right]=r+f=\widehat{d}_{H} .
$$

This is perfectly consistent with the $3 \mathrm{~d}$ mirror symmetry (2.34).

\footnotetext{
${ }^{12}$ Which may enhance to a larger, non-abelian flavor symmetry, in which case we are only considering the maximal torus.

${ }^{13}$ More precisely, we obtain the original electric quiver theory coupled to vector multiplets of opposite sign.

${ }^{14}$ This is what we called 'reduced HB' in table 1.
} 
By the same token, we may define the magnetic quiver of the $\mathcal{T}_{\mathbf{X}}^{5 \mathrm{~d}}$ as the gauging of the electric quiver for $\mathscr{T}_{\mathbf{X}}^{4 \mathrm{~d}}$ :

$$
\mathrm{MQ}^{(5)} \equiv \mathrm{EQ}^{(4)} / \mathrm{U}(1)^{f}
$$

Here, the $\mathrm{U}(1)^{f}$ flavor symmetry acts as an isometry of the Higgs branch $\mathcal{M}_{H}\left[\mathcal{T}_{\mathbf{X}}^{5 \mathrm{~d}}\right]$, and we have:

$$
\operatorname{dim} \mathrm{HB}\left[\mathrm{MQ}^{(5)}\right]=r, \quad \operatorname{dim} \mathrm{CB}\left[\mathrm{MQ}^{(5)}\right]=d_{H},
$$

in agreement with (2.35). These relations are simple tools to study the Higgs branch of either $\mathcal{T}_{\mathbf{X}}^{5 \mathrm{~d}}$ or $\mathscr{T}_{\mathbf{X}}^{4 \mathrm{~d}}$. They also relate many previously-known results to each other. In the following sections, we will demonstrate this in a few concrete examples.

\subsection{Higher-form symmetries of SCFTs}

The higher-form symmetries [124] of the SCFT $\mathcal{T}_{\mathbf{X}}^{5 \mathrm{~d}}$ or $\mathscr{T}_{\mathbf{X}}^{4 \mathrm{~d}}$, defined from the singularity $\mathbf{X}$, can be studied as in [79-81]. Here, we briefly discuss the case of $\mathbf{X}$ an isolated hypersurface singularity, and refer to [10] for a more detailed discussion.

The data of any QFT generally involves a choice of 'global structure' - for instance, for gauge theories, we need to choose a gauge group $G$, as opposed to only choosing a Lie algebra $\operatorname{Lie}(G)$; this choice translates into distinct spectra of Wilson lines and of $(d-3)$ dimensional 't Hooft operators [125]. In the geometric-engineering picture of the SCFT, the choice of global structure is related to a choice of consistent boundary conditions for the torsion fluxes in the non-compact threefold [79, 126].

A time-honored general strategy to study the SCFTs is through the analysis of its moduli space of vacua, which corresponds to resolving and/or deforming the singularity X. For instance, one can see the 'enhanced' flavor symmetry $G_{H}$ of the fixed point as the hyper-Kähler isometries of the conical Higgs branch. Similarly, one-form symmetries, or their magnetic $(d-3)$-form version for an $\mathrm{SCFT}_{d}$, can be studied on the Coulomb branch, where they are spontaneously broken [124]. We can also have $(d-2)$-form symmetries and their 'electro-magnetic' dual 0-form symmetries, which can be spontaneously broken on the Higgs branch.

In practice, one analyses the $q$-form symmetries by looking for $q$-dimensional charged operators that can obtain VEVs, in either the Higgs phase or the Coulomb phase. These operators are realized in string-theory as branes wrapping a relative 3-cycle of $\widehat{\mathbf{X}}$ which ends on a torsion 2-cycle of the boundary, $\partial \widehat{\mathbf{X}}$. We can then compute the higher-form symmetry [79-81] of the SCFT from the following relative homology group

$$
\text { Tor }\left[\operatorname{im}: H_{3}(\widehat{\mathbf{X}}, \partial \widehat{\mathbf{X}}, \mathbb{Z}) \rightarrow H_{2}(\widehat{\mathbf{X}}, \mathbb{Z})\right] \cong \operatorname{Tor} H_{2}\left(L_{5}(\mathbf{X}), \mathbb{Z}\right) \equiv \mathfrak{h}_{2}
$$

A choice of global structure corresponds to choosing a consistent half-dimensional sublattice of mutually-commuting fluxes. Note that, here, we always have:

$$
\mathfrak{h}_{2} \cong \mathfrak{f} \oplus \mathfrak{f},
$$

and that we will choose the simplest sub-lattices $(\mathfrak{f}, 0) \subset \mathfrak{h}_{2}$ or $(0, \mathfrak{f}) \subset \mathfrak{h}_{2}$, for simplicity, leaving a systematic analysis of the space of global structures for future work. We then have the following (non-exhaustive) list of possibilities, also summarized in table 2 : 


\begin{tabular}{|c|c|c|}
\hline SCFT $[\mathbf{X}]$ & 'electric' sym. / charged object & 'magnetic' sym. / charged object \\
\hline $\mathcal{T}_{\mathbf{X}}^{\text {d }}$ & $\Gamma_{e}^{(0)} /$ M2-brane & $\Gamma_{m}^{(3)} /$ M5-brane \\
$\mathscr{T}_{\mathbf{X}}^{4 \mathrm{~d}}$ & $\Gamma_{e}^{(1)} /$ D3-brane & $\Gamma_{m}^{(1)} /$ D3-brane \\
\hline
\end{tabular}

Table 2. The group $\mathfrak{f} \subset H_{2}\left(L_{5}(\mathbf{X}), \mathbb{Z}\right)$, interpreted as a $q$-form symmetry group $\Gamma^{(q)}$.

Three-form/zero-form symmetries of $\mathcal{T}_{\mathbf{X}}^{5 \mathrm{~d}}$. For the $5 \mathrm{~d}$ SCFT $\mathcal{T}_{\mathbf{X}}^{5 \mathrm{~d}}$, the defect group (2.44) corresponds to either M5-branes or M2-branes wrapping non-compact 3-cycles ending on a 2 -cycle in $\mathfrak{h}_{2}$. If we only allow for M5-branes in the spectrum, we have a $5 \mathrm{~d}$ theory with a 3 -form symmetry, while if we only allow M2-branes, we have a 0-form symmetry. Thus we have:

$$
\mathcal{T}_{\mathbf{X}}^{5 \mathrm{~d}}: \quad \Gamma_{m}^{(3)}=\mathfrak{f} \quad \text { or } \quad \Gamma_{e}^{(0)}=\mathfrak{f},
$$

for the 'magnetic' or 'electric' symmetry, respectively. These defect operators could be constructed in various ways in the field theory - similar codimension-2 defects in $5 \mathrm{~d}$ theories were constructed in [127]. We leave their analysis for future work.

Note that the SCFT $\mathcal{T}_{\mathbf{X}}^{5 \mathrm{~d}}$ arising from an hypersurface singularity does not have any one-form (or 2-form) symmetry [80,81], since the boundary $L_{5}(\mathbf{X})$ is simply-connected. This is contrast with the case of toric singularities, which we will briefly discuss in section 5 .

One-form symmetries of $\mathscr{T}_{\mathbf{X}}^{4 \mathrm{~d}}$. Similarly, in type-IIB string theory, we can wrap D3branes on non-compact 3 -cycles in $\widehat{\mathbf{X}}$. They correspond to line operators probing the Coulomb phase. We then have the one-form symmetry:

$$
\mathscr{T}_{\mathbf{X}}^{4 \mathrm{~d}}: \quad \Gamma_{m}^{(1)}=\mathfrak{f} \quad \text { or } \quad \Gamma_{e}^{(1)}=\mathfrak{f} .
$$

If $\mathscr{T}_{\mathbf{X}}^{4 \mathrm{~d}}$ is given by a superconformal Lagrangian, the global structure should simply correspond to the choice of gauge group $G$ - in particular, we will have a one-form symmetry if a discrete subgroup of the center, $\mathfrak{f} \subset Z(G)$, acts trivially on the matter fields. We will see examples of this below. For non-Lagrangian theories, on the other hand, such as generalized Argyres-Douglas (AD) theories, the analysis of the one-form symmetries will necessarily be more subtle. In fact, the string-theory engineering makes very specific predictions for the one-form symmetries of some AD theories, as we will discuss in section 4.4 below.

\section{5d SCFTs with Lagrangian 4d SCFT counterparts}

In this section, we illustrate the general discussion of the previous section with examples, where both sides of the $5 \mathrm{~d} / 4 \mathrm{~d}$ correspondence have a Lagrangian description. We start by discussing the $E_{8}$ rank-one $5 \mathrm{~d}$ SCFT [5], and its rank- $N$ generalizations [7], which already brings about interesting subtleties. We furthermore discuss a $5 \mathrm{~d}$ SCFT, which has an IR description in terms of a quiver theory, as well as the SCFT associated to $G_{2}+5 \boldsymbol{F}$. Each example illustrates different aspects. 


\begin{tabular}{|c|c|c|c|c|c|c|c|c|}
\hline$F$ & $r$ & $f$ & $d_{H}$ & $\widehat{r}$ & $\widehat{d}_{H}$ & $\Delta \mathcal{A}_{r}$ & $b_{3}$ & $\mathfrak{f}$ \\
\hline$x_{1}^{2}+x_{2}^{3}+x_{3}^{6}+x_{4}^{6 N}$ & $N$ & 6 & $12 N-1$ & $12 N-7$ & $N+6$ & $-N+1$ & $2 N-2$ & $\mathbb{Z}_{N}$ \\
$x_{1}^{2}+x_{2}^{4}+x_{3}^{4}+x_{4}^{4 N}$ & $N$ & 7 & $18 N-1$ & $18 N-8$ & $N+7$ & $-N+1$ & $2 N-2$ & $\mathbb{Z}_{N}$ \\
$x_{1}^{2}+x_{2}^{3}+x_{3}^{6}+x_{4}^{6 N}$ & $N$ & 8 & $30 N-1$ & $30 N-9$ & $N+8$ & $-N+1$ & $2 N-2$ & $\mathbb{Z}_{N}$ \\
\hline
\end{tabular}

Table 3. Isolated hypersurface singularities engineering the rank- $N E_{n}$ theories, for $n=6,7,8$.

\subsection{The rank- $N \quad E_{8}$ theory}

The famous rank-one $E_{n}$ SCFT [5], for $n \leq 8$, can be engineered in M-theory on a canonical singularity, $\mathbf{X}_{E_{n}}$, whose crepant resolution is the canonical line bundle over the del Pezzo surface $d P_{n} \cong \mathrm{Bl}_{n-1} \mathbb{P}^{1} \times \mathbb{P}^{1}[6]$. This resolution probes the extended Coulomb branch of the SCFT $\mathcal{T}_{\mathbf{X}_{E_{n}}}^{5 \mathrm{~d}}$, whose low-energy description is equivalent to the extended Coulomb branch of the $5 \mathrm{~d} \mathcal{N}=1 \mathrm{SU}(2)$ gauge theory with $N_{f}=n-1$ fundamental hypermultipets. These $5 \mathrm{~d}$ fixed points admit an interesting rank- $N$ generalization, with a gauge-theory phase:

$$
\operatorname{Sp}(N)+(n-1) \boldsymbol{F}+\boldsymbol{A} \boldsymbol{S} .
$$

For $N=1$, this reduces to the $\mathrm{Sp}(1) \cong \mathrm{SU}(2)+(n-1) \boldsymbol{F}$ gauge theory. The Higgs branch of the UV fixed point is expected to be the moduli space of $N E_{n}$ instantons in $\mathbb{C}^{2}$. For $n \geq 6$, these $5 \mathrm{~d}$ SCFTs can be engineered in M-theory on the following isolated hypersurface singularities:

$$
\begin{aligned}
& \mathbf{X}_{E_{6}}: \quad x_{1}^{3}+x_{2}^{3}+x_{3}^{3}+x_{4}^{3 N}=0, \\
& \mathbf{X}_{E_{7}}: x_{1}^{2}+x_{2}^{4}+x_{3}^{4}+x_{4}^{4 N}=0, \\
& \mathbf{X}_{E_{8}}: \quad x_{1}^{2}+x_{2}^{3}+x_{3}^{6}+x_{4}^{6 N}=0,
\end{aligned}
$$

up to some interesting subtleties for $N>1$, which we will discuss below. For $N=1$, in particular, each of these singularities admits a crepant resolution obtained by blowing up the origin of $\mathbb{C}^{4}, x_{1}=x_{2}=x_{3}=x_{4}=0$, by projective spaces with weights $(1,1,1,1)$, $(2,1,1,1)$ or $(3,2,1,1)$, respectively. The exceptional divisor is then $d P_{n}$ viewed as a hypersurface in (weighted) projective space.

Some of the basic properties of the singularities (3.2) are summarized in table 3, note that $b_{3}$ and $H_{2}\left(L_{5}, \mathbb{Z}\right)$ were also computed in [128]. In this section, we focus on the $E_{8}$ theories, for definiteness. The $E_{6}$ and $E_{7}$ families can be treated in exactly the same manner.

\subsubsection{Singularity spectrum and $4 \mathrm{~d}$ superconformal quiver}

Consider the singularity $\mathbf{X}_{E_{8}}$

$$
F(x)=x_{1}^{2}+x_{2}^{3}+x_{3}^{6}+x_{4}^{6 N}=0, \quad\left(q_{1}, q_{2}, q_{3}, q_{4}\right)=\left(\frac{1}{2}, \frac{1}{3}, \frac{1}{6}, \frac{1}{6 N}\right),
$$

with the scaling weights $q_{i}$ as indicated. The Milnor ring, $\mathcal{M}(F)$, of the corresponding isolated singularity, $\mathbf{X}=\{F=0\}$, is easily determined. One finds a non-trivial mixed Hodge structure on the level set of the singularity, with the dimensions (2.7) given by:

$$
f=\operatorname{dim} H^{2,2}(\widehat{\mathbf{X}})=8, \quad \widehat{r}=\operatorname{dim} H^{1,2}(\widehat{\mathbf{X}})=30 N-9,
$$


while the Milnor number is given by $\mu=2 \widehat{r}+f=60 N-10$. In particular, $H^{2,2}(\widehat{\mathbf{X}})$ is generated by the 8 deformations of $\mathbf{X}$ corresponding to the monomial in $x^{\mathfrak{m}} \in \mathcal{M}(F)$ with scaling dimension $Q=1-\frac{1}{6 N}$, namely:

$$
\begin{array}{llll}
x_{3} x_{4}^{5 N-1}, & x_{3}^{2} x_{4}^{4 N-1}, & x_{3}^{3} x_{4}^{3 N-1}, & x_{3}^{4} x_{4}^{2 N-1}, \\
x_{2} x_{4}^{4 N-1}, & x_{2} x_{3} x_{4}^{3 N-1}, & x_{2} x_{3}^{2} x_{4}^{2 N-1}, & x_{2} x_{3}^{3} x_{4}^{N-1} .
\end{array}
$$

In the IIB construction, they correspond to mass terms $-\mathrm{CB}$ operators of $\mathscr{T}_{\mathbf{X}}^{4 \mathrm{~d}}$ with scaling dimension $\Delta=1$. On general ground, as discussed in the previous section, $f$ is also the number of 'unpaired' 2 -cycles in the resolution $\widetilde{\mathbf{X}}$ of the singularity, so that we also have $f=85 \mathrm{~d} \mathcal{N}=1$ real mass deformations (sitting in background vector multiplets) apparent on the ECB of $\mathcal{T}_{\mathbf{X}}^{5 \mathrm{~d}}$. For $N>1$, there is an apparent mismatch with the number of real masses expected from the gauge-theory description (3.1)

$$
\operatorname{Sp}(N)+7 \boldsymbol{F}+\boldsymbol{A} \boldsymbol{S}
$$

from which one would expect 9 mass deformations ( 7 from the fundamentals, 1 from the antisymmetric, and 1 gauge coupling). Correspondingly, the $5 \mathrm{~d}$ global symmetry at the UV fixed point of (3.6) is expected to be $E_{8}$ for $N=1$, and $E_{8} \times \mathrm{SU}(2)$ for $N>1$. We will explain the reason for this discrepancy (when $N>1$ ) in subsection 3.1.2 below.

Let us first consider the $4 \mathrm{~d}$ SCFT $\mathscr{T}_{\mathbf{X}}^{4 \mathrm{~d}}$ engineered from the singularity (3.3) in IIB. In this case, as first shown in [103], the $4 \mathrm{~d}$ SCFT has a Lagrangian description as a superconformal gauge theory with gauge group:

$$
G=\prod_{d_{k}=\text { Dynkin label of } \widehat{E}_{8}} \mathrm{SU}\left(d_{k} N\right)
$$

where $d_{k}$ are the Dynkin labels of the affine $E_{8}$ algebra. This gauge group is coupled to hypermultiplets in bifundamental representations, giving us the $\widehat{E}_{8}$ gauge-theory quiver shown in figure 1. This result can be derived from the integer-valued singularity spectrum $\{\Delta\}$, as in [45]. For instance, for $N=1$, we have the following scaling dimensions of CB operators $(\Delta \geq 1)$ :

$$
\begin{array}{|l|l|l|l|l|l|l|}
\hline \Delta & 1 & 2 & 3 & 4 & 5 & 6 \\
\hline \# & 8 & 8 & 6 & 4 & 2 & 1 \\
\hline
\end{array}
$$

with their multiplicities indicated on the second line. Since an $\mathrm{SU}(K)$ gauge group contributes $\mathrm{CB}$ operators of dimension $\Delta=\{2,3, \cdots, K\}$, this spectrum is compatible with:

$$
G=\mathrm{SU}(6) \times \mathrm{SU}(5) \times \mathrm{SU}(4)^{2} \times \mathrm{SU}(3)^{2} \times \mathrm{SU}(2)^{2}, \quad \text { for } N=1 .
$$

This reasoning generalizes to $N>1$, giving us (3.7). There is then a unique way to couple all these gauge groups together with hypermultiplets to obtain an SCFT [129]. As a consistency check, we can compute the conformal anomalies of $\mathscr{T}_{\mathbf{X}}^{4 \mathrm{~d}}$ directly from the singularity spectrum, to obtain:

$$
n_{h}=-16 a+20 c=120 N^{2}, \quad n_{v}=8 a-4 c=120 N^{2}-9 .
$$




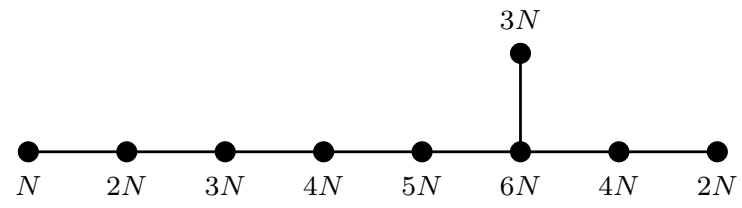

Figure 1. $4 \mathrm{~d} S U$-type $\mathcal{N}=2$ gauge-theory quiver for the rank-N $E_{8}$ theory. The same quiver graph describes the 'electric quiver' $\mathrm{EQ}^{(4)}$, seen as a $3 \mathrm{~d} \mathcal{N}=4$ theory.

This exactly matches the number of hypermultiplets and vector multiplets, respectively, in the $4 \mathrm{~d} \mathcal{N}=2$ gauge-theory quiver of figure 1 .

Since $\mathscr{T}_{\mathbf{X}}^{4 d}$ is a Lagrangian theory, its Higgs branch is computed semi-classically, as a hyper-Kähler quotient

$$
\mathcal{M}_{H}^{4 \mathrm{~d}} \cong \mathbb{C}^{240 N^{2}} / / / G
$$

For $N>1$, however, there is an interesting subtlety: a subgroup $\mathrm{U}(1)^{N-1} \subset G$ survives as free vector multiplets at low-energy. ${ }^{15}$ The Higgs branch quaternionic dimension is then:

$$
\widehat{d}_{H}=n_{h}-n_{v}+N-1=8+N .
$$

The $\mathrm{U}(1)_{r}$ 't Hooft anomaly of course match:

$$
24(c-a)=\widehat{d}_{H}-(N-1) .
$$

According to the general discussion in section 2.3, we should expect $2(N-1) 3$-cycles in the resolved geometry $\widetilde{\mathbf{X}}$. This is indeed the case, as we will see momentarily. The $4 \mathrm{~d}$ $\mathcal{N}=2$ theory of figure 1 also has $\mathbb{Z}_{N}$ one-form symmetry, in agreement with the prediction from the geometry.

\subsubsection{Resolutions: 5d Coulomb branch and 4d Higgs branch}

From the singular equation (3.3) for $\mathbf{X}$, we can explicitly construct the crepant resolution $\pi: \widetilde{\mathbf{X}} \rightarrow \mathbf{X}$. Using the notation of $[21,131]$, the resolution is obtained as a sequence of weighted blow-ups (taking the proper transform) in the ambient space:

$$
\begin{aligned}
& \left(x_{1}^{(3)}, x_{2}^{(2)}, x_{3}^{(1)}, x_{4}^{(1)} ; \delta_{1}\right), \\
& \left(x_{1}^{(3)}, x_{2}^{(2)}, x_{3}^{(1)}, \delta_{i}^{(1)} ; \delta_{i+1}\right), \quad \text { for } i=1, \cdots, N-1 .
\end{aligned}
$$

\footnotetext{
${ }^{15}$ This is rather familiar in a different context: this $\widehat{E}_{8} 4 \mathrm{~d} \mathcal{N}=2$ quiver also arises as the SCFT at low-energy on the worldvolume of $N$ D3-branes probing the (resolved) orbifold of $\mathbb{C}^{2}$ by the ' $E_{8}$ ' finite subgroup of $\mathrm{SU}(2), Y=\mathbb{C}^{2} / \Gamma_{E_{8}}$ [130]. In that language, the Higgs branch of the $\mathcal{N}=2$ quiver corresponds to the 8 resolution parameters of the orbifold singularity (the so-called baryonic operators), $\pi: \widetilde{Y} \rightarrow Y$, and to the $N$ positions of the D3-branes on $\widetilde{Y}$ (the so-called mesonic operators). The low-energy U(1) vector multiplets are the degrees of the freedom of the $N$ separated probe D3-branes (modulo the center-of-mass $\mathrm{U}(1)$, which is decoupled).
} 
The superscripts denote the weights of the blowup, and $\delta_{i}$ are the sections associated to the exceptional divisors of the blowup. The fully resolved singularity is completely smooth can be summarized by the equation:

$$
\widetilde{\mathbf{X}}: \quad x_{1}^{2}+x_{2}^{3}+x_{3}^{6}+x_{4}^{6 N} \prod_{i=1}^{N} \delta_{i}^{6 N-6 i}=0 .
$$

If $N=1$, the single exceptional divisor, $S_{1} \cong\left\{\delta_{1}=0\right\}$, is a smooth degree-6 hypersurface in the weighted projective space $\mathbb{P}^{3,2,1,1}$, which exactly has the topology of $d P_{8}$. This confirms that the $5 \mathrm{~d}$ SCFT $\mathcal{T}_{\mathbf{X}}^{5 \mathrm{~d}}$ is the rank-one $E_{8}$ theory, with the IR gauge-theory description $\mathrm{SU}(2)+7 \boldsymbol{F}[6]$.

For $N \geq 2$, the triple intersection numbers among the $N$ exceptional divisors $S_{i}$ are:

$$
\begin{aligned}
S_{1}^{3}=S_{2}^{3}=\cdots=S_{N-1}^{3} & =0, & S_{N}^{3} & =1, \\
S_{i}^{2} \cdot S_{i-1} & =-1, & S_{i-1}^{2} \cdot S_{i} & =1, \quad(i=2, \ldots, N),
\end{aligned}
$$

with all other intersection numbers vanishing. From the Riemann-Roch theorem and the adjunction formula:

$$
S_{1}^{2} \cdot S_{2}+S_{1} \cdot S_{2}^{2}=2 g\left(S_{1} \cdot S_{2}\right)-2,
$$

one finds that the intersection curves $S_{i} \cdot S_{i+1}(i=1, \ldots, N-1)$ all have genus $g\left(S_{i} \cdot S_{i+1}\right)=1$. In fact, the surfaces $S_{i}, i<N$, are ruled surface over a genus-1 curve, $\mathbb{P}^{1} \rightarrow S_{i} \rightarrow T^{2}$, while $S_{N}$ is a $d P_{8}$ surface. These intersection numbers are exactly the ones obtained for the gauge theory (3.6) engineered in [7]. ${ }^{16}$ The Hodge diamond of the surface $S_{k}$, for $k<N$, reads:

$$
h^{i, j}\left(S_{k}\right)=\left(\begin{array}{lll}
1 & 1 & 0 \\
1 & 2 & 1 \\
0 & 1 & 1
\end{array}\right), \quad k=1, \cdots, N-1 .
$$

In particular, we find a pair of 3-cycles on each of these surface. Therefore, in total, there are $2(N-1) 3$-cycles among all the $S_{k}$. This agrees with the number of 3 -cycles in the smooth local threefold $\widetilde{\mathbf{X}}$, which can be computed using the methods of [100]:

$$
b_{3}=H_{3}(\widetilde{\mathbf{X}}, \mathbb{Z})=2 N-2 .
$$

Note that, on the other hand, the space $\widetilde{\mathbf{X}}$ is simply connected.

When $N \geq 2$, interestingly, one can go through a geometric transition that flops the genus-1 ruled surfaces $S_{i}(i<N)$ into Hirzebruch surfaces, and blows up the surface $S_{N}$ (which becomes $\mathrm{Bl}_{8} \mathbb{F}_{3}$ ). (For the case of $N=2$, this flop was explicitly constructed in $[16,21]$.) After this transition, the $(N-1)$-intersection curves $S_{i} \cdot S_{i+1}$ all become genuszero curves. As a consequence, all the 3-cycles disappear and there is one additional 2cycle in the flopped geometry, $\widetilde{\mathbf{X}}_{\text {flopped }}$. Alternatively, this geometric transition can also be understood from the $(p, q)$-web for the $5 \mathrm{~d} \operatorname{Sp}(N)$ gauge theory, as we discuss in appendix $\mathrm{C}$.

\footnotetext{
${ }^{16}$ See equations (8.13)-(8.14) of [7], plugging in $g=0, g^{\prime}=1$ and $n_{F}=7$.
} 
Interpretation in $\mathcal{T}_{\mathrm{X}}^{5 \mathrm{~d}}$ : the ' $\boldsymbol{A S}$ hypermultiplet phase transition.' The resolved geometry (3.15) should correspond to a generic point on the extended Coulomb branch of the 5d SCFT $\mathcal{T}_{\mathbf{X}_{E_{8}}}^{5 \mathrm{~d}}$. As already mentioned, from the gauge theory perspective (3.6), one expects the ECB to be of real dimension $N+9$, instead of:

$$
d_{H}=r+f=N+8,
$$

as dictated by the geometry. Recall that a hypermultiplet in a representation $\mathfrak{R}$ of the $5 \mathrm{~d}$ gauge group $G_{5 \mathrm{~d}}$ contributes to the prepotential as [7]:

$$
\mathcal{F}_{\mathfrak{R}}=-\frac{1}{12} \sum_{\rho \in \mathfrak{R}}\left|\rho(\varphi)+m_{\mathfrak{R}}\right|^{3},
$$

where the sum is over all the weights $\rho$ of the representation, $\varphi$ denotes the CB VEVs of $G_{5 \mathrm{~d}}$ and $m_{\mathfrak{R}}$ is a real 'flavor' mass. For the antisymmetric of $\operatorname{Sp}(N)$, we have:

$$
\mathcal{F}_{\boldsymbol{A} \boldsymbol{S}}=-\frac{N-1}{12}\left|m_{\boldsymbol{A} \boldsymbol{S}}\right|^{3}-\frac{1}{12} \sum_{\rho \neq 0}\left|\rho(\varphi)+m_{\boldsymbol{A} \boldsymbol{S}}\right|^{3} .
$$

The key point is that the $\boldsymbol{A S}$ of $\operatorname{Sp}(N)$, of dimension $(2 N+1)(N-1)$, has $N-1$ vanishing weights, $\rho=(0, \cdots, 0)$. Therefore, at a generic point on the $\operatorname{Sp}(N)$ Coulomb branch, we have $N-1$ neutral hypermultiplets of mass $m_{\boldsymbol{A} S}$. In the limit $m_{\boldsymbol{A} S} \rightarrow 0$, these neutral hypermultiplets can be given a VEV.

This phase transition resolves our puzzle. In M-theory, we interpret the resolved geometry (3.15) as the Coulomb branch of the SCFT in a 'partial Higgs phase,' which can be described as the $\operatorname{Sp}(N)$ gauge theory on its CB with $m_{A S}=0$ and non-zero VEVs for the $N-1$ neutral hypermultiplets, as we just discussed. In this phase, the $5 \mathrm{~d}$ SCFT flavor symmetry will be:

$$
G_{H}^{5 \mathrm{~d}}=E_{8},
$$

for any $N$, as we will see. The geometric transition mentioned above corresponds to turning off these VEVs and turning on the mass for the antisymmetric, $m_{\boldsymbol{A} S} \neq 0$, which corresponds to the new Kähler parameter for the additional curve in $S_{N} \subset \widetilde{\mathbf{X}}_{\text {flopped }}$ after the flop. One could then, in principle, go to the origin of the Kähler cone of this flopped geometry, where we would expect an SCFT with flavor symmetry $E_{8} \times \mathrm{SU}(2)$. For $N=2$, this flavor symmetry is manifest in the model derived from a non-isolated singularity [21]; however, to our knowledge the geometry that makes the $\mathrm{SU}(2)$ manifest for $N>2$ is unknown. It would be interesting to find such explicit geometries, including as isolated singularities if they exist.

In summary, our puzzle in the counting of the mass parameters of $\mathcal{T}_{\mathbf{X}}^{5 \mathrm{~d}}$ arose as an order-of-limit issue. If we consider $\mathcal{T}_{\mathbf{X}}^{5 \mathrm{~d}}$ as defined by the canonical singularity (3.3), there is no issue except that the 5 d gauge-theory interpretation (3.1) is partially lost. ${ }^{17}$ If we

\footnotetext{
${ }^{17}$ Note that is not in contradiction with [7], where the prepotential of the $\operatorname{Sp}(N)+7 \boldsymbol{F}+\boldsymbol{A} \boldsymbol{S}$ gauge theory was matched to the prepotential computed from the intersection numbers (3.16), because that computation was not keeping track of the mass parameters. Instead, one should redo these computations while keeping track of all the non-compact divisors, as done recently in [15, 132] for toric singularities, and in [21] for elliptic models.
} 
insist on interpreting this SCFT as a 'strong-coupling limit' (i.e. a UV completion) of the $5 \mathrm{~d} \mathcal{N}=1$ gauge theory, the correct interpretation is that one needs to first send $m_{A S}$ to zero, go to the 'partial Higgs phase' for the chargeless hypermultiplets, then take a scaling limit wherein all the remaining $N+8$ ECB parameters are sent to zero.

\subsubsection{Magnetic quivers and the 5d Higgs branch}

The $4 \mathrm{~d} \mathcal{N}=2$ quiver of figure 1 can be directly reduced to $3 \mathrm{~d}$, since it is a Lagrangian SCFT. Thus, we see that the 'electric quiver' $\mathrm{MQ}^{(4)}$ of $\mathscr{T}_{\mathbf{X}}^{4 \mathrm{~d}}$ is just the $\widehat{E}_{8}$ quiver with $\mathrm{SU}(N)$ gauge group, now viewed as a $3 \mathrm{~d} \mathcal{N}=4$ gauge theory. This theory (like $\mathscr{T}_{\mathbf{X}}^{4 \mathrm{~d}}$ itself) has a flavor symmetry $\mathrm{U}(1)^{8}$, which are simply the symmetries rotating the 8 bifundamental hypermultiplets. Gauging this symmetry according to our general prescription, we obtain the magnetic quiver of the $5 \mathrm{~d}$ SCFT

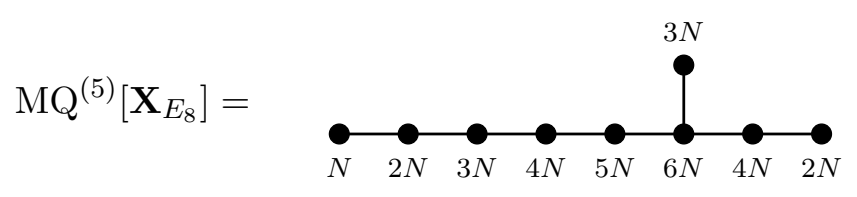

with the gauge group $\prod_{k} \mathrm{U}\left(d_{k} N\right)$ modulo the diagonal $\mathrm{U}(1)$. The $\mathrm{CB}$ of this $\mathrm{MQ}^{(5)}$ can be studied by standard methods. We see, in particular, that its has the expected dimension:

$$
d_{H}=\operatorname{dim} \mathcal{M}_{H}^{5 \mathrm{~d}}=\widehat{r}+f=30 N-1 .
$$

Moreover, this $\mathrm{MQ}^{(5)}$ implies that $\mathcal{T}_{\mathbf{X}}^{5 \mathrm{~d}}$ has the enhanced flavor symmetry $E_{8}$; the Cartan of this $E_{8}$ is the topological symmetry $\mathrm{U}(1)^{8}$ of the unitary quiver. For $N>1$, the $\mathrm{MQ}^{(5)}$ (3.24) is distinct from the one expected from the strong-coupling limit of the $5 \mathrm{~d}$ $\operatorname{Sp}(N)$ theory, for the reasons explained above. The latter quiver would be:

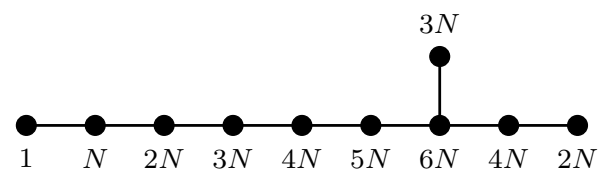

whose CB gives the (centered) moduli space of $N$ instantons - see e.g. [133]. Note that the additional U(1) node, which renders the node adjacent to it 'unbalanced,' could not come from some Lagrangian matter in a 4d SCFT, since that would break four-dimensional conformal invariance.

Finally, let us highlight the fact that we have derived the magnetic quiver (3.24) directly from the singularity $\mathbf{X}_{E_{8}}$, without using $(p, q)$-webs. The same $\mathrm{MQ}^{(5)}$ can be obtained from a standard web construction for the $\operatorname{Sp}(N)$ theory in its 'partial Higgs phase', as explained in appendix C.

\subsection{Rank-two $5 \mathrm{~d}$ SCFT with a gauge-theory phase $\mathrm{SU}(2)_{0}-\mathrm{SU}(2)-5 \mathrm{~F}$}

As another interesting example, consider the canonical singularity:

$$
F(x)=x_{1}^{2}+x_{2}^{5}+x_{3}^{10}+x_{3} x_{4}^{3}=0, \quad\left(q_{1}, q_{2}, q_{3}, q_{4}\right)=\left(\frac{1}{2}, \frac{1}{5}, \frac{1}{10}, \frac{3}{10}\right) .
$$




\begin{tabular}{|c|c|c|c|c|c|c|c|c|}
\hline$F$ & $r$ & $f$ & $d_{H}$ & $\widehat{r}$ & $\widehat{d}_{H}$ & $\Delta \mathcal{A}_{r}$ & $b_{3}$ & $\mathfrak{f}$ \\
\hline$x_{1}^{2}+x_{2}^{5}+x_{3}^{10}+x_{3} x_{4}^{3}$ & 2 & 8 & 46 & 38 & 10 & 0 & 0 & 0 \\
\hline$x_{1}^{2}+x_{2}^{5}+x_{3}^{5}+x_{4}^{5}$ & 2 & 0 & 32 & 32 & 2 & -6 & 12 & $\mathbb{Z}_{2}^{6}$ \\
\hline
\end{tabular}

Table 4. The isolated singularities studied in subsections 3.2 and 3.3, respectively.

Its basic properties are summarized on the first line of table 4 . As we will show, this singularity engineers an SCFT $\mathcal{T}_{\mathbf{X}}^{5 \mathrm{~d}}$ which admits a gauge-theory phase:

$$
\mathrm{SU}(2)_{0}-\mathrm{SU}(2)-5 \boldsymbol{F} .
$$

The enhanced symmetry at the UV fixed point is known to be $G_{H}^{5 \mathrm{~d}}=E_{8}$, through other methods $[21,134,135]$. Let us analyse this model in the language of this paper.

\subsubsection{Singularity spectrum and $4 \mathrm{~d}$ superconformal quiver}

The Milnor ring of the singularity (3.27) has $\mu=84$ generators. Amongst these, there are exactly eight generators corresponding to $4 \mathrm{~d} \mathcal{N}=2 \mathrm{CB}$ operators with scaling dimension $\Delta=1$ (that is, scaling weight $Q=\frac{9}{10}$ ):

$$
x_{3}^{6} x_{4}, x_{3}^{9}, x_{2} x_{3}^{4} x_{4}, x_{2} x_{3}^{7}, x_{2}^{2} x_{3}^{2} x_{4}, x_{2}^{2} x_{3}^{5}, x_{2}^{3} x_{4}, x_{2}^{3} x_{3}^{3} .
$$

We thus find $f=8$, which matches the rank of the flavor symmetry (including the topological symmetries) of the $5 \mathrm{~d}$ gauge theory (3.28). In addition, there are $\widehat{r}=38$ generators with $\Delta>1$, corresponding to the $\mathrm{CB}$ operators of $\mathscr{T}_{\mathbf{X}}^{4 \mathrm{~d}}$, whose ECB spectrum reads:

$$
\begin{array}{|r|r|r|r|r|r|r|r|r|r|r|}
\hline \Delta & 1 & 2 & 3 & 4 & 5 & 6 & 7 & 8 & 9 & 10 \\
\hline \# & 8 & 8 & 7 & 7 & 5 & 4 & 3 & 2 & 1 & 1 \\
\hline
\end{array}
$$

This spectrum is compatible with a $4 \mathrm{~d}$ gauge group:

$$
G=\mathrm{SU}(10) \times \mathrm{SU}(8) \times \mathrm{SU}(7) \times \mathrm{SU}(6) \times \mathrm{SU}(5) \times \mathrm{SU}(4)^{2} \times \mathrm{SU}(2) .
$$

We can also compute the conformal anomalies of $\mathscr{T}_{\mathbf{X}}^{4 \mathrm{~d}}$ or, equivalently, the effective number of hypermultiplets and vector multiplets:

$$
n_{h}=312, \quad n_{v}=302 .
$$

It is then easy to see that the $4 \mathrm{~d}$ SCFT is the $\mathcal{N}=2$ superconformal Lagrangian quiver shown in figure 2, with gauge group (3.31). The 4d Higgs branch then determined semiclassically. In particular, the four-dimensional HB dimension is:

$$
\widehat{d}_{H}=n_{h}-n_{v}=10 .
$$

The $4 \mathrm{~d} \mathcal{N}=2$ quiver directly reduces to the electric quiver $\mathrm{EQ}^{(4)}$, described by the same gauge theory with $3 \mathrm{~d} \mathcal{N}=4$ vector multiplets. By gauging the $\mathrm{U}(1)^{8}$ flavor symmetry 


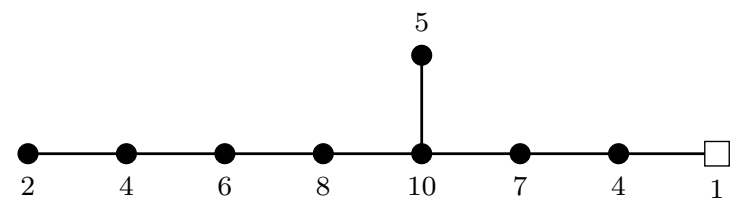

Figure 2. The $\mathcal{N}=2 \operatorname{SCFT} \mathscr{T}_{\mathbf{X}}^{4 \mathrm{~d}}$ for the singularity $x_{1}^{2}+x_{2}^{5}+x_{3}^{10}+x_{3} x_{4}^{3}=0$, where each node is an $\mathrm{SU}(K)$ gauge group, with $K$ as indicated.

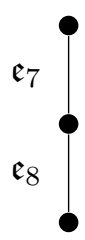

Figure 3. Hasse diagram for $\mathcal{T}_{\mathbf{X}}^{5 \mathrm{~d}}$ defined by $x_{1}^{2}+x_{2}^{5}+x_{3}^{10}+x_{3} x_{4}^{3}=0$, with magnetic quiver (3.34).

rotating the bifundamental hypermultiplets in $\mathrm{EQ}^{(4)}$, we obtain the magnetic quiver of the five-dimensional SCFT, $\mathcal{T}_{\mathbf{X}}^{5 \mathrm{~d}}$, as a unitary quiver given by

$$
\mathrm{MQ}^{(5)}=
$$

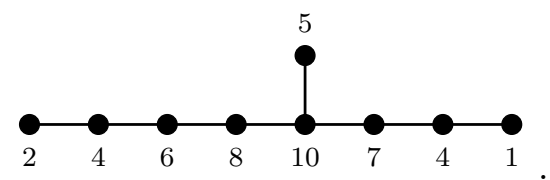

From this magnetic quiver, we can see that the flavor symmetry of $\mathcal{T}_{\mathbf{X}}^{5 \mathrm{~d}}$ enhances to $E_{8}$ at the fixed point. This is apparent in the quiver itself, which has balanced nodes in the shape of an $E_{8}$ Dynkin diagram, and it can also be seen by using the quiver subtraction technique [30] to obtain the Hasse diagram of the Higgs branch $H B\left[\mathcal{T}_{\mathbf{X}}^{5 \mathrm{~d}}\right]$, shown in figure 3 . In fact, the magnetic quiver (3.34) exactly corresponds to the next-to-minimal nilpotent orbit of $E_{8}$, as studied in [136]. The $5 \mathrm{~d}$ HB dimension (computed as the dimension of $\left.\mathrm{CB}\left[\mathrm{MQ}^{(5)}\right]\right)$ also matches the counting of generators in the Milnor ring, $d_{H}=\widehat{r}+f=46$, by construction.

\subsubsection{Resolved geometry and 5d Coulomb branch}

Given the $4 \mathrm{~d}$ Higgs branch dimension (3.33), $\mathcal{T}_{\mathbf{X}}^{5 \mathrm{~d}}$ should have rank $r=\widehat{d}_{H}-f=2$. This can be seen explicitly, by resolving the singularity (3.27). The resolution sequence is:

$$
\begin{aligned}
& \left(x_{1}^{(2)}, x_{2}^{(1)}, x_{3}^{(1)}, x_{4}^{(1)} ; \delta_{1}\right), \\
& \left(x_{1}^{(3)}, x_{4}^{(2)}, x_{2}^{(1)}, \delta_{1}^{(1)} ; \delta_{2}\right),
\end{aligned}
$$

which gives us a smooth threefold:

$$
\widetilde{\mathbf{X}}: x_{1}^{2}+x_{2}^{5} \delta_{1}+x_{3}^{10} \delta_{1}^{6}+x_{3} x_{4}^{3}=0 .
$$


The exceptional divisors, $S_{1} \cong\left\{\delta_{1}=0\right\}$ and $S_{2} \cong\left\{\delta_{2}=0\right\}$, are irreducible, thus the $\mathrm{CB}$ dimension of $\mathcal{T}_{\mathbf{X}}^{5 \mathrm{~d}}$ is indeed given by $r=2$. We can also compute the following triple intersection numbers:

$$
S_{1}^{3}=1, \quad S_{2}^{3}=1, \quad S_{1}^{2} \cdot S_{2}=1, \quad S_{1} \cdot S_{2}^{2}=-1 .
$$

The exceptional divisor $S_{1}$ is a singular surface, however:

$$
\delta_{1}=0: x_{1}^{2}+x_{3} x_{4}^{3}=0 .
$$

It can be viewed as ruled over a singular cubic curve $x_{1}^{2}+x_{4}^{3}=0$, with a cusp at $x_{1}=x_{4}=0$. In this case, we can perform a flop by blowing up this singular cubic at the double point on $S_{2}$ - see [16, 21] for more details and examples. After the flop, we have the following intersection numbers:

$$
S_{1}^{\prime 3}=9, \quad S_{2}^{\prime 3}=0, \quad S_{1}^{\prime 2} \cdot S_{2}^{\prime}=-3, \quad S_{1}^{\prime} \cdot S_{2}^{\prime 2}=1 .
$$

Here, $S_{1}^{\prime}$ has the topology of $\mathbb{P}^{2}$ and $S_{2}^{\prime}$ is a Hirzebruch surface $\mathbb{F}_{3}$ blown up at eight points. After another flop (blowing up $S_{1}$ and blowing down $S_{2}$ ), the intersection numbers match the ones for the rank-two gauge theory $(3.28)[16,21]$.

Note that there is no 3 -cycle in the resolved geometry $\widetilde{\mathbf{X}}$, because the genus-1 curve $S_{1}$. $S_{2}$ is a nodal curve and the 1 -cycle has collapsed. Hence we have $b_{3}=0$. (The same result is obtained with the methods of [100].) This model has no higher-form symmetry, either.

\subsection{Rank-two 5d SCFT with a gauge-theory phase $G_{2}+5 \mathrm{~F}$}

As our third example, consider the isolated singularity:

$$
F(x)=x_{1}^{2}+x_{2}^{5}+x_{3}^{5}+x_{4}^{5}=0, \quad\left(q_{1}, q_{2}, q_{3}, q_{4}\right)=\left(\frac{1}{2}, \frac{1}{5}, \frac{1}{5}, \frac{1}{5}\right) .
$$

whose properties are summarized on the last line of table 4. It turns out that it engineers an SCFT with IR gauge theory descriptions

$$
\mathrm{SU}(3)_{\frac{9}{2}}+5 \boldsymbol{F}, \quad \mathrm{Sp}(2)+3 \boldsymbol{F}+2 \boldsymbol{A} \boldsymbol{S}, \quad G_{2}+5 \boldsymbol{F} .
$$

The enhanced flavor symmetry at the UV fixed point is $G_{H}^{5 \mathrm{~d}}=\operatorname{Sp}(6)$ [21]. Similarly to the $\mathrm{SU}(2)$ flavor symmetry factor for the rank- $N E_{8}$ theory of subsection 3.1, this flavor symmetry turns out to be rather subtle from the point of view of the isolated singularity. We make some preliminary comments on this issue below, and defer a complete discussion to future work.

\subsubsection{Singularity spectrum and $4 \mathrm{~d}$ superconformal quiver}

There are $\mu=64$ generators in the Milnor ring of the singularity (3.40). The $4 \mathrm{~d}$ ECB spectrum, corresponding to monomials with dimension $\Delta \geq 1$, is as follows:

$$
\begin{array}{|l|l|l|l|l|l|}
\hline \Delta & 2 & 4 & 6 & 8 & 10 \\
\hline \# & 12 & 10 & 6 & 3 & 1 \\
\hline
\end{array}
$$


Note that there is mass term (corresponding to $\Delta=1$ ), in this case, and that all the scaling dimension are even. This gives us:

$$
f=0, \quad d_{H}=\widehat{r}=32 .
$$

The spectrum (3.42) and the conformal anomalies:

$$
n_{h}=232, \quad n_{v}=236,
$$

can be matched by a Lagrangian $4 \mathrm{~d} \mathcal{N}=2 \mathrm{SCFT}$, which takes the form the orthosymplectic quiver shown in figure $4 .{ }^{18}$ From the resolution $\widetilde{\mathbf{X}}$, to be discussed below, we find $r=2$ and therefore:

$$
\widehat{d}_{H}=n_{h}-n_{v}+6=2 .
$$

In particular, there should be 6 massless vector at low energy on the Higgs branch of the orthosymplectic quiver of figure 4. Note that this identification is not unique, and as will be discussed in [137], this particular CB spectrum has an alternative quiver realization.

Relatedly, this $4 \mathrm{~d} \mathcal{N}=2$ quiver has a $\mathbb{Z}_{2}^{6}$ one-form symmetry, arising from the fact that the fundamental of $\operatorname{Spin}(K)$ preserves the $\mathbb{Z}_{2}$ center; this matches perfectly with the geometry of the link, since $H_{2}\left(L_{5}(\mathbf{X}), \mathbb{Z}\right)=\mathbb{Z}_{2}^{12}$.

Since $f=0$, the orthosymplectic quiver reduced to $3 \mathrm{~d}$ directly gives us the magnetic quiver of $\mathcal{T}_{\mathbf{X}}^{5 \mathrm{~d}}$, namely:

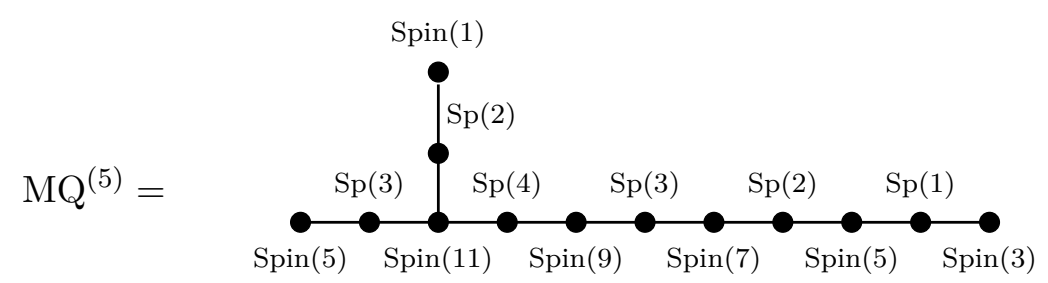

Of course, its Coulomb branch dimension matches $d_{H}=\widehat{r}=32$, by construction. It would be very interesting to study the Coulomb branch of (3.46) further, for instance by computing its Hilbert series.

\subsubsection{Resolved geometry and 5d Coulomb branch}

Given the singular equation (3.40), we apply the following resolution sequence:

$$
\begin{aligned}
& \left(x_{1}^{(2)}, x_{2}^{(1)}, x_{3}^{(1)}, x_{4}^{(1)} ; \delta_{1}\right), \\
& \left(x_{1}, \delta_{1} ; \delta_{2}\right), \\
& \left(\delta_{1}, \delta_{2} ; \delta_{3}\right) .
\end{aligned}
$$

The resulting space is smooth

$$
\widetilde{\mathbf{X}}: \quad x_{1}^{2} \delta_{2}+\left(x_{2}^{5}+x_{3}^{5}+x_{4}^{5}\right) \delta_{1}=0 .
$$

\footnotetext{
${ }^{18}$ This particular quiver also appeared in [129]. In fact, the general discussion of that paper is very useful in order to identify the possible $\mathcal{N}=2$ superconformal Lagrangian (if any) associated to a given $\mathrm{CB}$ spectrum. Note that the rank- $K$ gauge groups $\mathrm{Sp}(K)$ and $\mathrm{SO}(2 K+1)$ both have $\mathrm{CB}$ dimensions $\Delta=\{2,4, \cdots, 2 K\}$.
} 


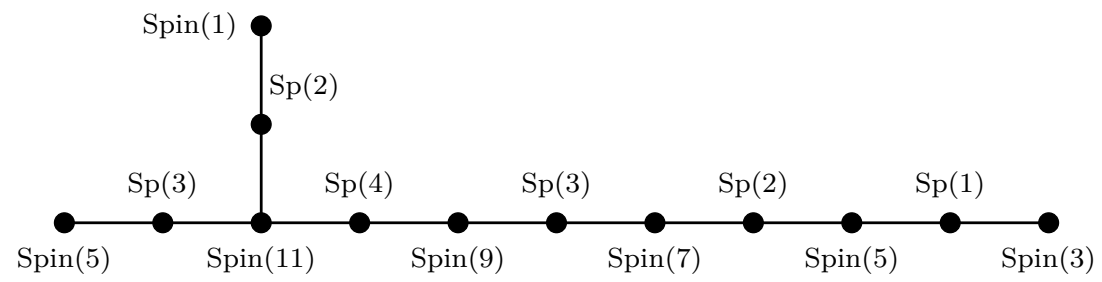

Figure 4 . The $4 \mathrm{~d} \mathcal{N}=2$ superconformal quiver for the singularity $x_{1}^{2}+x_{2}^{5}+x_{3}^{5}+x_{4}^{5}=0$. Here, the links are hypermultiplets in the 'bifundamental' $(\boldsymbol{m}, \boldsymbol{k})$ of $\operatorname{Sp}(m) \times \operatorname{Spin}(k)$.

As one can check, the set $\left\{\delta_{1}=0\right\}$ is empty. There are thus only two exceptional divisors $S_{1} \cong\left\{\delta_{2}=0\right\}$ and $S_{2} \cong\left\{\delta_{3}=0\right\}$, which are both irreducible. Therefore, the 5d SCFT $\mathcal{T}_{\mathbf{X}}^{5 \mathrm{~d}}$ has rank $r=2$. The triple intersection numbers are:

$$
S_{1}^{3}=-40, \quad S_{2}^{3}=9, \quad S_{1}^{2} \cdot S_{2}=25, \quad S_{2}^{2} \cdot S_{1}=-15 .
$$

Using (3.17), we can see that $S_{1} \cdot S_{2}$ is a genus-6 curve. In fact, $S_{1}$ is ruled over the genus-6 curve $S_{1} \cdot S_{2}$. As $S_{2}$ has the topology of a $\mathbb{P}^{2}$, the $S_{1} \cdot S_{2}$ is also exactly the degree-5 curve on $\mathbb{P}^{2}$ with genus $g=6$. This makes sense because the curve $S_{1} \cdot S_{2}$ has equation $\delta_{2}=\delta_{3}=x_{2}^{5}+x_{3}^{5}+x_{4}^{5}=0$, which has degree five. The Hodge diamond of $S_{1}$ takes the form:

$$
h^{i, j}\left(S_{1}\right)=\left(\begin{array}{lll}
1 & 6 & 0 \\
6 & 2 & 6 \\
0 & 6 & 1
\end{array}\right) .
$$

Thus, there are 123 -cycles on $S_{1}$, which also correspond to 123 -cycles in the resolved threefold [100]. These 3 -cycles give rise 6 vector multiplets on the Higgs branch of $\mathscr{T}_{\mathbf{X}}^{4 \mathrm{~d}}$, as in (3.45).

Note that, in the M-theory engineering, these 3-cycles must also corresponds to 6 neutral hypermultiplets obtaining a VEV on the Coulomb branch of $\mathcal{T}_{\mathbf{X}}^{5 \mathrm{~d}}$. Unlike for the case of the higher-rank $E_{8}$ theory of section 3.1, it is not immediately clear whether this 'partial Higgs phase' can be understood directly from the gauge-theory description (3.41).

To connect this resolved geometry to the $5 \mathrm{~d}$ gauge theory, we have to go through a geometric transition: we pinch off six points on the genus-6 curve $S_{1} \cdot S_{2}$, and blow up these six double points on $S_{2}$. After these flops, the triple intersection numbers of the new surfaces $S_{1}^{\prime}, S_{2}^{\prime}$ will be

$$
S_{1}^{\prime 3}=8, \quad S_{2}^{\prime 3}=3, \quad S_{1}^{\prime 2} \cdot S_{2}^{\prime}=1, \quad S_{2}^{\prime 2} \cdot S_{1}^{\prime}=-3 .
$$

Hence $S_{1}^{\prime}$ is a $\mathbb{F}_{3}$ and $S_{2}^{\prime}$ is a $d P_{6}$. These triple intersection numbers exactly give rise to the 5d SCFT with the IR gauge-theory description (3.41). Before the flop, our resolved geometry has $f=0$, in agreement with the singularity spectrum. However, after the six blow-ups of double points, there are six new 2-cycles in the flopped geometry, while all the 3-cycles have disappeared. These six new Kähler parameters then match the rank of the expected UV fixed point of the gauge theories (3.41), which has flavor symmetry $G_{F}=\operatorname{Sp}(6)$. 
Clearly, a better understanding of this apparent phase transition on the Coulomb branch of the $5 \mathrm{~d}$ SCFT $\mathcal{T}_{\mathbf{X}}^{5 \mathrm{~d}}$ is desirable, both in terms of the gauge theory description and in terms of the local Calabi-Yau.

\section{Argyres-Douglas theories and their 5d SCFT counterparts}

In the Type IIB setup, the most studied $4 \mathrm{~d}$ fixed points are the (generalized) ArgyresDouglas (AD) theories [84] engineered at the hypersurface singularities [8, 42]

$$
F\left(x_{1}, x_{2}, x_{3}, x_{4}\right)=g_{G}\left(x_{1}, x_{2}\right)+g_{G^{\prime}}\left(x_{3}, x_{4}\right)=0,
$$

where the two-variable polynomial $g_{G}(x, y)$ defines the ADE singularity

$$
\begin{array}{llrl}
g_{A_{k}}(x, y) & =x^{2}+y^{k+1}, & g_{D_{k}}(x, y) & =x^{k+1}+x y^{2}, \\
g_{E_{6}}(x, y) & =x^{3}+y^{4}, & g_{E_{7}}(x, y) & =x^{3}+x y^{3},
\end{array}
$$

We will denote these so-called $\left(G, G^{\prime}\right)$ theories [42] by

$$
\mathrm{AD}\left[G, G^{\prime}\right] \equiv \mathscr{T}_{\left[G, G^{\prime}\right]}^{4 \mathrm{~d}} .
$$

Of course, one can study many other SCFTs in a similar manner, such as, for instance, the $4 \mathrm{~d}$ SCFTs that arise at exceptional unimodal singularities [138] which are not of the $\left[G, G^{\prime}\right]$ type [139]. Here, we focus on the class (4.1) for definiteness; see [10] for more examples.

We display a few examples, with their basic properties, in table 5. Note that many of these canonical singularities do not admit crepant blow ups, so that $r=0 .{ }^{19}$ From our general discussion, they must therefore correspond to 'rank-zero' $5 \mathrm{~d}$ SCFTs $\mathcal{T}_{\mathbf{X}}^{5 \mathrm{~d}}$. We will see that this is indeed the case, although it is not yet clear whether the rank-zero $\mathcal{T}_{\mathbf{X}}^{5 \mathrm{~d}}$ is a 'trivial' theory - in the sense that it could consists of free 5 d hypermultiplets 'in disguise'.

We will also discuss an interesting example with $r=1$, corresponding to a singularity that engineers a non-trivial, higher-rank SCFT both in 4d and $5 \mathrm{~d}$.

\subsection{4d fixed-points with $r=0$ and 3d mirrors}

Let us first review some well-known fact about some of these AD theories with $r=0$, in this IIB engineering perspective [8, 42, 43].

The theory $\operatorname{AD}\left[\boldsymbol{A}_{1}, \boldsymbol{A}_{\mathbf{2}-1}\right]$. This series includes the free hypermultiplet for $N=1$, since $\mathbf{X}_{\left[A_{1}, A_{1}\right]}$ is the conifold singularity. We have:

$$
f=1, \quad \widehat{r}=N-1, \quad \widehat{d}_{H}=1 .
$$

The $3 \mathrm{~d}$ mirror (that is, $\mathrm{MQ}^{(4)}$ ) is known to be $3 \mathrm{~d} \mathcal{N}=4 \operatorname{SQED}\left[N_{f}=N\right]$; equivalently, it is a $\mathrm{U}(1) \times \mathrm{U}(1)$ quiver with $N$ bifundamentals, modulo the decoupled diagonal $\mathrm{U}(1)$ [86]

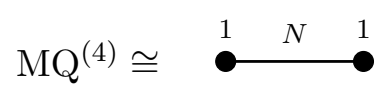

\footnotetext{
${ }^{19}$ The singularities (4.1) have $r=0$ for $\left(G, G^{\prime}\right)=\left(A_{k}, G^{\prime}\right)$, for any $k$ and any $G$ of type $A, D$ or $E$.
} 


\begin{tabular}{|c|c|c|c|c|c|c|c|c|c|c|c|}
\hline$\left[G, G^{\prime}\right]$ & $F$ & $r$ & $f$ & $d_{H}$ & $\widehat{r}$ & $\widehat{d}_{H}$ & $a$ & $c$ & $\Delta \mathcal{A}_{r}$ & $\mu$ & $b_{3}$ \\
\hline$\left[A_{1}, A_{1}\right]$ & $x_{1}^{2}+x_{2}^{2}+x_{3}^{2}+x_{4}^{2}$ & 0 & 1 & 1 & 0 & 1 & $\frac{1}{24}$ & $\frac{1}{12}$ & 0 & 1 & 0 \\
{$\left[A_{1}, A_{2}\right]$} & $x_{1}^{2}+x_{2}^{2}+x_{3}^{2}+x_{4}^{3}$ & 0 & 0 & 1 & 1 & 0 & $\frac{43}{120}$ & $\frac{11}{30}$ & $\frac{1}{5}$ & 2 & 0 \\
{$\left[A_{1}, A_{3}\right]$} & $x_{1}^{2}+x_{2}^{2}+x_{3}^{2}+x_{4}^{4}$ & 0 & 1 & 2 & 1 & 1 & $\frac{11}{24}$ & $\frac{1}{2}$ & 0 & 3 & 0 \\
{$\left[A_{1}, A_{4}\right]$} & $x_{1}^{2}+x_{2}^{2}+x_{3}^{2}+x_{4}^{5}$ & 0 & 0 & 2 & 2 & 0 & $\frac{67}{84}$ & $\frac{17}{21}$ & $\frac{2}{7}$ & 4 & 0 \\
\hline$\left[A_{1}, D_{5}\right]$ & $x_{1}^{2}+x_{2}^{2}+x_{3}^{4}+x_{3} x_{4}^{2}$ & 0 & 1 & 3 & 2 & 1 & $\frac{19}{20}$ & 1 & $\frac{1}{5}$ & 5 & 0 \\
{$\left[A_{1}, D_{6}\right]$} & $x_{1}^{2}+x_{2}^{2}+x_{3}^{5}+x_{3} x_{4}^{2}$ & 0 & 2 & 4 & 2 & 2 & $\frac{13}{12}$ & $\frac{7}{6}$ & 0 & 6 & 0 \\
{$\left[A_{1}, D_{7}\right]$} & $x_{1}^{2}+x_{2}^{2}+x_{3}^{6}+x_{3} x_{4}^{2}$ & 0 & 1 & 4 & 3 & 1 & $\frac{81}{56}$ & $\frac{3}{2}$ & $\frac{2}{7}$ & 7 & 0 \\
{$\left[A_{1}, D_{8}\right]$} & $x_{1}^{2}+x_{2}^{2}+x_{3}^{7}+x_{3} x_{4}^{2}$ & 0 & 2 & 5 & 3 & 2 & $\frac{19}{12}$ & $\frac{5}{3}$ & 0 & 8 & 0 \\
\hline$\left[A_{2}, A_{2}\right]$ & $x_{1}^{2}+x_{2}^{3}+x_{3}^{2}+x_{4}^{3}$ & 0 & 2 & 3 & 1 & 2 & $\frac{7}{12}$ & $\frac{2}{3}$ & 0 & 4 & 0 \\
{$\left[A_{3}, A_{3}\right]$} & $x_{1}^{2}+x_{2}^{4}+x_{3}^{2}+x_{4}^{4}$ & 0 & 3 & 6 & 3 & 3 & $\frac{15}{8}$ & 2 & 0 & 9 & 0 \\
{$\left[A_{4}, A_{4}\right]$} & $x_{1}^{2}+x_{2}^{5}+x_{3}^{2}+x_{4}^{5}$ & 0 & 4 & 10 & 6 & 4 & $\frac{25}{6}$ & $\frac{13}{3}$ & 0 & 16 & 0 \\
\hline$\left[A_{2}, D_{4}\right]$ & $x_{1}^{2}+x_{2}^{3}+x_{3}^{3}+x_{3} x_{4}^{2}$ & 0 & 0 & 4 & 4 & 0 & 2 & 2 & 0 & 8 & 0 \\
\hline
\end{tabular}

Table 5. $5 \mathrm{~d}$ and $4 \mathrm{~d}$ data for some singularities giving rise to $\mathscr{T}_{\mathbf{X}}^{4 \mathrm{~d}}=\mathrm{AD}\left[G, G^{\prime}\right]$. Here, $a$ and $c$ are the conformal anomalies of $\mathscr{T}_{\mathbf{X}}^{4 \mathrm{~d}}$. Note also that $\left[A_{2}, A_{2}\right] \cong\left[A_{1}, D_{4}\right]$.

The Higgs branch is

$$
\mathcal{M}_{H}^{4 \mathrm{~d}} \cong \mathbb{C}^{2} / \mathbb{Z}_{N} \cong \mathrm{CB}\left[\mathrm{MQ}^{(4)}\right]
$$

Of course, for $N=1$, we recover the elementary $3 \mathrm{~d}$ mirror symmetry between a hypermultiplet and $\operatorname{SQED}\left[N_{f}=1\right]$. The Higgs branch (4.6) corresponds to the small resolution of the singularity:

$$
x_{1}^{2}+x_{2}^{2}+x_{3}^{2}+x_{4}^{2 N}=0,
$$

which leaves us with a smooth local CY threefold with a single Kähler parameter (for $N=1$, this is the resolved conifold).

The theory $\operatorname{AD}\left[\boldsymbol{A}_{1}, \boldsymbol{A}_{2 N}\right]$. In this case, one has:

$$
f=0, \quad \widehat{r}=N, \quad \widehat{d}_{H}=0 .
$$

Therefore the Higgs branch is trivial. The CB spectrum is $\Delta=\left\{\frac{2 N+2+2 j}{2 N+3}\right\}_{j=1}^{N}$. It has been proposed that the $3 \mathrm{~d}$ mirror consist of $N$ free hypermultiplets [140, 141]:

$$
\mathrm{MQ}^{(4)} \cong(\text { hyper })^{\otimes N} \text {. }
$$

From the IIB construction, we see that the HB is empty because the terminal singularity:

$$
x_{1}^{2}+x_{2}^{2}+x_{3}^{2}+x_{4}^{2 N+1}=0
$$

does not admit any (small or otherwise) crepant resolution. This is also reflected in the $\operatorname{Tr}\left(\mathrm{U}(1)_{r}\right)$ anomaly, $24(c-a)=\frac{N}{2 N+3}$, which is not integer. 
The theory $\operatorname{AD}\left[A_{1}, D_{2 N+2}\right]$. In this case, one finds:

$$
f=2, \quad \widehat{r}=N, \quad \widehat{d}_{H}=2 .
$$

The $3 \mathrm{~d}$ mirror is given by the following quiver (modulo the diagonal $\mathrm{U}(1)$, so the gauge group is $\left.\mathrm{U}(1)^{2}\right)$ :

$$
\mathrm{MQ}^{(4)}=\underbrace{1}_{1}
$$

The Higgs branch $\mathcal{M}_{H}^{4 \mathrm{~d}} \cong \mathrm{CB}\left[\mathrm{MQ}^{(4)}\right]$ has quaternionic dimension 2. The flavor symmetry $G_{H}^{4 \mathrm{~d}}$ is $\mathrm{SU}(3)$ for $N=1$ and $\mathrm{SU}(2) \times \mathrm{U}(1)$ for $N>1$.

The theory $\mathrm{AD}\left[\boldsymbol{A}_{1}, D_{2 N+1}\right]$. In this case, one finds:

$$
f=1, \quad \widehat{r}=N, \quad \widehat{d}_{H}=1 .
$$

This Higgs branch is $\mathbb{C}^{2} / \mathbb{Z}_{2}$ for any $N$. In addition, the low-energy theory on the Higgs branch also contains the irreducible $\operatorname{SCFT} \mathrm{AD}\left[A_{1}, A_{2 N-2}\right]$, which itself has no Higgs branch. The $3 \mathrm{~d}$ mirror is expected to consists of a tensor product of $\operatorname{SQED}\left[N_{f}=2\right]$ (whose CB realizes the $\mathbb{C}^{2} / \mathbb{Z}_{2}$ Higgs branch) with $(N-1)$ free hypermultiplets:

$$
\mathrm{MQ}^{(4)} \cong(\text { hyper })^{\otimes(N-1)} \otimes \operatorname{SQED}\left[N_{f}=2\right],
$$

as argued recently in [141]. The canonical singularity:

$$
x_{1}^{2}+x_{2}^{2}+x_{3}^{2 N+2}+x_{3} x_{4}^{2}=0
$$

admits a small resolution which leaves us with the residual terminal singularity that gives the IR theory $\operatorname{AD}\left[A_{1}, A_{2 N-2}\right]$.

The theory $\operatorname{AD}\left[\boldsymbol{A}_{k}, \boldsymbol{A}_{\boldsymbol{k}}\right]$. This family of theories is particularly interesting. We have:

$$
f=k, \quad \widehat{r}=\frac{k(k-1)}{2}, \quad \widehat{d}_{H}=k .
$$

The $3 \mathrm{~d}$ mirror is the complete graph with $k+1$ nodes $[86,142]$ :

$$
\mathrm{MQ}^{(4)}\left[\mathbf{X}_{\left[A_{1}, A_{1}\right]}\right]=
$$


modulo the diagonal $\mathrm{U}(1)$. Note that $\mathrm{AD}\left[A_{2}, A_{2}\right] \cong \mathrm{AD}\left[A_{1}, D_{4}\right]$. From the magnetic quivers, we also learn that the flavor group $G_{H}^{4 \mathrm{~d}}$ for $\mathrm{AD}\left[A_{1}, D_{4}\right]$ is $\mathrm{SU}(3)$, while the flavor symmetry for $k>2$ is $\mathrm{U}(1)^{k}$. These singularities admit a smooth resolution, with the exceptional locus consisting of $k$ rational curves, therefore we have a smooth Higgs branch phase. This family also generalizes to $\mathrm{AD}\left[A_{k}, A_{k N+N-1}\right]$, whose $3 \mathrm{~d}$ mirrors are the same complete graphs (4.17) but with all links of multiplicity $N$.

Interestingly, we can also access the 'electric quiverine' directly in certain cases, as follows. The $\mathrm{AD}\left[A_{k}, A_{k}\right]$ theory has $k-2$ marginal couplings, which can be tuned to reach an S-duality cusp [143], from which we can read off some useful 'quasi-Lagrangian' description. Such descriptions consist of $\mathrm{SU}(n)$ gauge groups coupled together with strongly-coupled 'matter' $[144,145]$, which can be reduced to $3 d$ 'sequentially' at weak coupling. As a simple example, consider $\mathrm{AD}\left[A_{3}, A_{3}\right]$, which has an $S$-dual description [146]:

$$
\mathrm{AD}\left[A_{3}, A_{3}\right]=\quad \overbrace{D_{4}-\mathrm{SU}(2)-D_{4}}^{1}
$$

Here, an $\mathrm{SU}(2)$ subgroup of the $D_{4}$ theory (that is, $\left[A_{1}, D_{4}\right]$ ) is gauged in conformal manner; indeed, the CB spectrum of $\left[A_{3}, A_{3}\right]$ is $\Delta=\left\{\frac{3}{2}, \frac{3}{2}, 2\right\}$, and $D_{4}$ is rank- 1 with $\Delta=\frac{3}{2}$. Since $D_{4}$ itself flows to the $\operatorname{SQED}\left[N_{f}=3\right]$ fixed point in $3 \mathrm{~d}$, by weakly gauging the $\mathrm{SU}(2)$, one finds the $3 \mathrm{~d}$ description:

$$
\mathrm{EQ}^{(4)}\left[\mathbf{X}_{\left[A_{3}, A_{3}\right]}\right]=\underset{1}{\longrightarrow} \mathrm{SU}(2) \stackrel{1}{\bullet}
$$

This must be a mirror description of the bottom quiver in (4.17), as recently discussed in $[145]$.

\subsection{5d rank-zero fixed points and their magnetic quivers}

We would like to understand better the $5 \mathrm{~d}$ SCFTs $\mathcal{T}_{\mathbf{X}}^{5 \mathrm{~d}}$ arising from these same singularities. These theories have $r=0$ and $f$ mass deformations, corresponding to $f$ small-resolution Kähler parameters. These mass deformations trigger an RG flow, which ends on the trivial theory (with $f$ background gauge fields, from M-theory on the 2-cycles of $\widetilde{\mathbf{X}}$ ) if the small resolution $\widetilde{\mathbf{X}}$ is smooth. However, we might be left with a terminal singularity, such as, for instance, in (4.10), which would signal that we flowed to a simpler 'irreducible' SCFT $\mathcal{T}_{\mathbf{X}}^{5 \mathrm{~d}}$ without massive deformations. This is the interpretation of the partial resolution of (4.15) in M-theory, in particular.

The singularity $\mathbf{X}\left[\boldsymbol{A}_{1}, \boldsymbol{A}_{2 N-1}\right] . \quad$ For $N=1$, the conifold, we know that $\mathcal{T}_{\mathrm{X}}^{5 \mathrm{~d}}$ is the free $5 \mathrm{~d}$ hypermultiplet, while $\mathscr{T}_{\mathbf{X}}^{4 \mathrm{~d}}$ is the free $4 \mathrm{~d}$ hypermultiplet; we have $f=1$. The electric theory for both the $5 \mathrm{~d}$ and the $4 \mathrm{~d}$ theory is the free hyper in $3 \mathrm{~d}$, while the magnetic quivers are SQED with one electron:

$$
\mathrm{EQ}^{(5)} \cong \mathrm{EQ}^{(4)} \cong \text { hyper }, \quad \mathrm{MQ}^{(5)} \cong \mathrm{MQ}^{(4)} \cong \operatorname{SQED}\left[N_{f}=1\right]
$$


Of course, the electric and magnetic theories are related by mirror symmetry, and at the same time the gauging of the $\mathrm{U}(1)$ flavor of the electric theory gives the correct result, as in (2.37) and (2.42).

Next, consider the case $N=2$. The magnetic quiver $\mathrm{MQ}^{(4)}(4.5)$ is self-mirror in this case, so that:

$$
\operatorname{EQ}^{(4)}\left[\mathbf{X}_{\left[A_{1}, A_{3}\right]}\right]=\operatorname{SQED}\left[N_{f}=2\right],
$$

with the flavor symmetry $\mathrm{U}(1) \subset \mathrm{SU}(2)_{F}$ acting on the two hypermultiplets with charge \pm 1 . Using the gauging prescription (2.42), we get the magnetic quiver of $\mathcal{T}_{\mathbf{X}}^{5 \mathrm{~d}}$ described by a $\mathrm{U}(1) \times \mathrm{U}(1)$ gauge theory coupled to two hypermultiplets with charges:

$$
\mathrm{MQ}^{(5)}\left[\mathbf{X}_{\left[A_{1}, A_{3}\right]}\right]: \quad \quad \begin{array}{|c|cc|}
\hline & H_{1} & H_{2} \\
\hline \mathrm{U}(1)_{1} & 1 & 1 \\
\mathrm{U}(1)_{2} & 1 & -1 \\
\hline
\end{array}
$$

This $3 \mathrm{~d}$ theory obviously has a Higgs branch of dimension zero, while its Coulomb is of quaternionic dimension 2, which by construction gives us the Higgs branch of $\mathcal{T}_{\mathbf{X}}^{5 \mathrm{~d}}$. To gain a better understanding of the structure of the $3 \mathrm{~d}$ quantum $\mathrm{CB}$, we may compute its Hilbert series using the monopole formula [49]. One finds:

$$
\mathrm{HS}_{\mathrm{CB}[\mathrm{MQ}}^{(5)]}(t)=\frac{1+6 t^{2}+t^{4}}{\left(1-t^{2}\right)^{4}} .
$$

This is consistent with $\mathcal{M}_{H}\left[\mathcal{T}_{\mathbf{X}}^{5 \mathrm{~d}}\right]$ being a hyper-Kähler cone of dimension $d_{H}=2$. From the plethystic logarithm $\mathrm{PL}[\mathrm{HS}]=10 t^{2}-20 t^{4}+\cdots$, we see that the $5 \mathrm{~d}$ HB is a non-complete intersection in $\mathbb{C}^{10}$, and from the small-t expansion $\mathrm{HS}=1+10 t^{2}+\cdots$, we find that the theory has 10 conserved currents [147, 148]. Indeed, this Higgs branch is the minimal nilpotent orbit of $\operatorname{Sp}(2)$, and appears as a $\mathbb{Z}_{2}$ gauging of two hypermultiplets [133]. Thus we have the flavor group $G_{H}^{5 \mathrm{~d}}=\operatorname{Sp}(2)$. We provide some more details on the Hilbert series computations in appendix A.

The careful reader ${ }^{20}$ will have noticed that this larger symmetry group seems in contradiction with our general discussion of the global symmetry as seen from the (resolved) geometry in M-theory. In fact, such unexpected 'enhanced symmetries', where even the rank of $G_{H}^{5 \mathrm{~d}}$ is larger than expected, can often happen when $\mathcal{T}_{\mathbf{X}}^{5 \mathrm{~d}}$ contains a free-hypermultiplet sector (modulo discrete gauging), which is the case here. We will discuss this interesting state of affairs in more detail elsewhere.

The singularity $\mathbf{X}\left[\boldsymbol{A}_{1}, \boldsymbol{A}_{\mathbf{2}}\right]$. Given the $3 \mathrm{~d}$ mirror $\mathrm{MQ}^{(4)}$ and the fact that $f=0$, we would conclude that the rank-zero $5 \mathrm{~d} \operatorname{SCFT} \mathcal{T}_{\mathbf{X}_{\left[A_{1}, A_{2 N}\right]}^{5 \mathrm{~d}}}$ flows to $N$ hypers upon compactification on a torus.

The singularity $\mathbf{X}\left[\boldsymbol{A}_{\mathbf{1}}, \boldsymbol{D}_{\mathbf{4}}\right] \cong \mathbf{X}\left[\boldsymbol{A}_{\mathbf{2}}, \boldsymbol{A}_{\mathbf{2}}\right]$. This should give an $r=0, d_{H}=35 \mathrm{~d}$ theory. The $3 \mathrm{~d}$ mirror of the $\mathrm{MQ}^{(4)}(4.12)$ with $N=1$ is simply:

$$
\mathrm{EQ}^{(4)}\left[\mathbf{X}_{\left[A_{2}, A_{2}\right]}\right]=\operatorname{SQED}\left[N_{f}=3\right]
$$

\footnotetext{
${ }^{20}$ And a thorough anonymous JHEP referee.
} 
We now gauge the $\mathrm{U}(1)^{2}$ maximal torus of the $\mathrm{SU}(3)$ flavor symmetry to obtain the 'magnetic quiver' theory:

$$
\mathrm{MQ}^{(5)}\left[\mathbf{X}_{\left[A_{2}, A_{2}\right]}\right]: \quad \quad \begin{array}{ccccc}
\mathrm{U}(1)_{1} & 1 & 1 & 1 \\
\mathrm{U}(1)_{2} & 1 & -1 & 0 \\
\mathrm{U}(1)_{3} & 0 & -1 & 1 \\
\hline
\end{array}
$$

with gauge group $\mathrm{U}(1)^{3}$, three hypermultiplets and a zero-dimensional Higgs branch. We have $d_{H}=3$, with the Hilbert Series:

$$
\mathrm{HS}_{\mathrm{CB}\left[\mathrm{MQ}^{(5)}\right]}(t)=\frac{1-3 t+12 t^{2}-11 t^{3}+12 t^{5}-3 t+t^{6}}{(1-t)^{6}\left(1+t+t^{2}\right)^{3}}=1+9 t^{2}+30 t^{3}+\cdots .
$$

The singularity $\mathbf{X}\left[\boldsymbol{A}_{\boldsymbol{k}}, \boldsymbol{A}_{\boldsymbol{k}}\right]$. The corresponding 5d SCFTs have:

$$
r=0, \quad f=k, \quad d_{H}=\frac{k(k+1)}{2} .
$$

We studied the case $k=2$ above, and the cases $k>2$ could be studied similarly; this is left for future work. Here, let us simply mention that, for $\left[A_{3}, A_{3}\right]$, we can also directly obtain a magnetic quiver for $\mathcal{T}_{\mathbf{X}}^{5 \mathrm{~d}}$ by gauging the obvious $\mathrm{U}(1)^{3}$ flavor symmetry in (4.19):

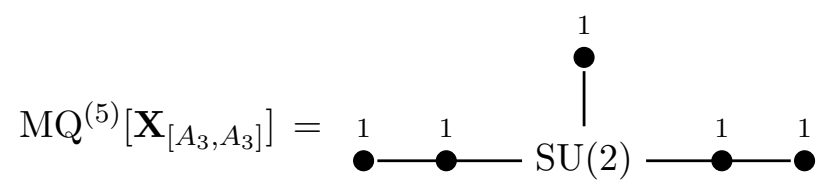

This quiver indeed has a trivial Higgs branch, and a Coulomb branch of dimension $d_{H}=6$.

An interesting feature of this particular family of singularities is that we also know its 5d BPS quiver - the D-brane quiver for $D_{S^{1}} \mathcal{T}_{\mathbf{X}}^{5 \mathrm{~d}}$ in IIA [149]. It consists of a loop of $k+1$ nodes connected by pairs of bifundamental arrows in both directions with a quartic superpotential, generalizing the Klebanov-Witten quiver [150] $(k=1)$ to $k>1$ [151]. It would be very interesting to study the BPS spectrum of $D_{S^{1}} \mathcal{T}_{\mathbf{X}}^{5 \mathrm{~d}}$ for $k>1$ along the lines of [149].

\subsection{Further examples: higher-rank 5d theories}

Let us also mention a couple of other examples with interesting features. The first example involves a non-trivial choice of global structure, while the second example has non-zero rank, $r>0$, in $5 \mathrm{~d}$.

\subsubsection{The $\mathrm{X}_{\left[A_{2}, D_{4}\right]}$ theory}

This singularity has $r=0, f=0$ and $d_{H}=4$. The singularity can be equivalently written as:

$$
x_{1}^{2}+x_{2}^{3}+x_{3}^{3}+x_{4}^{3}=0
$$


The $4 \mathrm{~d}$ SCFT has $\widehat{r}=4$ with the spectrum $\Delta=\left\{2, \frac{4}{3}, \frac{4}{3}, \frac{4}{3}\right\}$ - thus, one marginal coupling - and $\widehat{d}_{H}=0$. We then propose that this theory can be described as the conformal gauging of three $\mathrm{AD}\left[A_{1}, A_{3}\right]$ theories:

$$
\mathrm{AD}\left[A_{2}, D_{4}\right]=\left.\quad\right|_{A_{3}-\mathrm{SU}(2)-A_{3}} ^{A_{3}} \quad \text { or }\left.\quad\right|_{A_{3}-\mathrm{SO}(3)_{+}-A_{3}} ^{A_{3}}
$$

From the geometry, we can compute $H_{2}\left(L_{5}\left(\mathbf{X}_{\left[A_{2}, D_{4}\right]}, \mathbb{Z}\right)=\mathbb{Z}_{2}^{2}\right.$, so that the theory $\mathscr{T}_{\mathbf{X}}^{4 \mathrm{~d}}$ has either an electric or a magnetic $\mathbb{Z}_{2}$ one-form symmetry; we propose that the charged line operators are precisely the Wilson or 't Hooft lines in the gauge-theory description (4.30). (Note that we can also have $\mathrm{SO}(3)_{-}$, with the discrete $\theta$-angle turned on [125], by choosing the diagonal lattice in $(2.45) .{ }^{21}$ )

Changing the gauge coupling in (4.30) corresponds to deforming the singularity (4.29) by the monomial $x_{2} x_{3} x_{4}$. It is tempting to take a singular limit to the singularity $x_{1}^{2}+$ $x_{2} x_{3} x_{4}=0$, which is the toric singularity $\mathbb{C}^{3} /\left(\mathbb{Z}_{2} \times \mathbb{Z}_{2}\right)$, also known as the $5 \mathrm{~d} T_{2}$ model [121]. From the fact that $A_{3}$ reduces to $\operatorname{SQED}\left[N_{f}=2\right]$ in $3 \mathrm{~d}$, we infer:

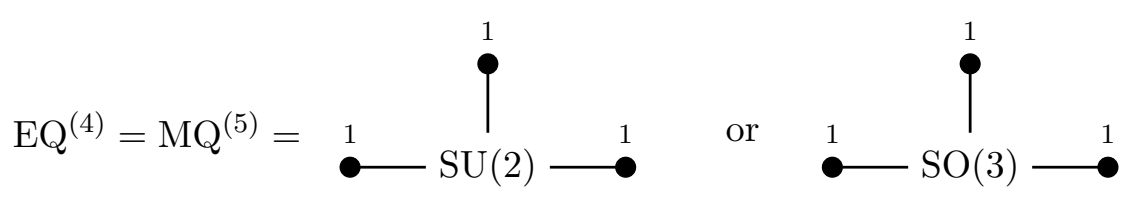

which must also be equal to $\mathrm{MQ}^{(5)}$ since $f=0$. This agrees with a direct application of the rules of [78] for computing $\mathrm{MQ}^{(5)}$ for the toric singularity. Thus, we propose that the SCFT $\mathcal{T}_{\mathbf{X}}^{5 \mathrm{~d}}$ related to the $\left[A_{2}, D_{4}\right]$ Argyres-Douglas theory in IIB is precisely the $5 \mathrm{~d} T_{2}$. Thus, the mirror of (4.31) should be given by 4 hypermultiplets. In fact, the quiver (4.31) is essentially 'ugly' in the technical sense [147], which implies the presence of free fields in the IR, up to discrete gauging. By computing the CB Hilbert series for (4.31) (see appendix A.1), we find the HS of $\mathcal{M}_{H}^{5 \mathrm{~d}}$ for either choice of the global structure:

$$
\mathrm{HS}_{\mathrm{SU}(2)}=\frac{1+28 t^{2}+70 t^{4}+28 t^{6}+t^{8}}{\left(1-t^{2}\right)^{8}}, \quad \quad \mathrm{HS}_{\mathrm{SO}(3)}=\frac{1}{(1-t)^{8}},
$$

where we indicated the choice of gauge group in (4.31). We see that $\mathcal{T}_{\mathbf{X}}^{5 \mathrm{~d}}$ has a Higgs branch $\mathbb{C}^{8}$ in the $\mathrm{SO}(3)$ gauging case. In the $\mathrm{SU}(2)$ gauging case, we obtain the HS of the minimal nilpotent orbit of $\operatorname{Sp}(4)$ [152], consistent with a $\mathbb{Z}_{2}$ gauging of four hypermultiplets.

\subsubsection{Example of a rank-one 5d SCFT coupled to a rank-zero theory}

Our geometric setup also leads to many more examples of 5d SCFTs with $r>0$, which are, in some sense, obtained by 'coupling' the rank-zero theories to higher-rank theories. While a detailed study of such a 'coupling' is beyond the scope of this paper, we present one such example here. (See [10] for many more examples.)

\footnotetext{
${ }^{21}$ This corresponds to lines realized by M2/M5-brane dyonic bound states.
} 


\begin{tabular}{|c|c|c|c|c|c|c|c|c|}
\hline$F$ & $r$ & $f$ & $d_{H}$ & $\widehat{r}$ & $\widehat{d}_{H}$ & $\Delta \mathcal{A}_{r}$ & $b_{3}$ & $\mathfrak{f}$ \\
\hline$x_{1}^{3}+x_{2}^{3}+x_{3}^{3}+x_{4}^{5}$ & 1 & 0 & 16 & 16 & 1 & -1 & 2 & $\mathbb{Z}_{5}$ \\
\hline
\end{tabular}

Table 6. The SCFT data associated to the singularity $x_{1}^{3}+x_{2}^{3}+x_{3}^{3}+x_{4}^{5}=0$.

This example is a rank-1 $5 \mathrm{~d}$ SCFT coupled to the $\mathbf{X}_{\left[A_{2}, D_{4}\right]}$ theory mentioned in the last section. The resulting theory is an apparently new rank-1 SCFT, not part of the $E_{n}$ series discovered by Seiberg $[5,6]$, and it also provides evidence that the $5 \mathrm{~d}$ rank-0 SCFT $\mathcal{T}_{\mathbf{X}}^{5 \mathrm{~d}}{ }_{\left[A_{2}, D_{4}\right]}$ is non-trivial as a 'matter component'. This rank-one SCFT is defined as the low-energy limit of M-theory at the isolated hypersurface singularity $\mathbf{X}$ given by:

$$
F(x)=x_{1}^{3}+x_{2}^{3}+x_{3}^{3}+x_{4}^{5}=0 .
$$

The resolution is a weighted blow up with trivial weight $(1,1,1,1)$, which is exactly the same as for the rank-1 $E_{6}$ theory:

$$
\left(x_{1}, x_{2}, x_{3}, x_{4} ; \delta_{1}\right)
$$

The resolved equation:

$$
\widetilde{\mathbf{X}}: x_{1}^{3}+x_{2}^{3}+x_{3}^{3}+x_{4}^{5} \delta_{1}^{2}=0,
$$

has a terminal singularity of type $\mathbf{X}_{\left[A_{2}, D_{4}\right]}$ at $\delta_{1}=x_{1}=x_{2}=x_{3}=0$ :

$$
\delta_{1}^{2}+x_{1}^{3}+x_{2}^{3}+x_{3}^{3}=0 .
$$

Thus the $5 \mathrm{~d} \operatorname{SCFT} \mathcal{T}_{\mathbf{X}}^{5 \mathrm{~d}}$ on $\mathbf{X}$ can be interpreted as a non-trivial 'coupling' of a rank-1 $E_{6}$ theory with the $\mathbf{X}_{\left[A_{2}, D_{4}\right]}$ theory. Note that, if we interpret the same resolved geometry as the Higgs branch of the $4 \mathrm{~d}$ SCFT $\mathscr{T}_{\mathbf{X}}^{4 \mathrm{~d}}$, the interpretation is rather mundane: we have a residual $4 \mathrm{~d}$ SCFT $\operatorname{AD}\left[A_{2}, D_{4}\right]$ at every point on the one-dimensional Higgs branch of the larger theory, which happens to be $\operatorname{AD}\left[D_{4}, E_{8}\right]$. On the other hand, the most straightforward interpretation in M-theory is rather more provocative: we have a rank-1 5d SCFT with an enhanced Coulomb branch (in the sense of [153]) — that is, there are additional light degrees of freedom at every point on the CB.

From the deformation and resolution of $\mathbf{X}$, we can compute the relevant data of $\mathcal{T}_{\mathbf{X}}^{5 \mathrm{~d}}$ and $\mathscr{T}_{\mathbf{X}}^{4 \mathrm{~d}}$, as shown in table 6 . As one can see, the flavor rank of $\mathcal{T}_{\mathbf{X}}^{5 \mathrm{~d}}$ is bounded by $\operatorname{rank}\left(G_{H}^{5 \mathrm{~d}}\right) \leq f+\frac{1}{2} b_{3}=1$, and the HB dimension is $d_{H}=16$. More interestingly, there exists a non-trivial 3-form symmetry $\Gamma_{m}^{(3)}=\mathbb{Z}_{5}$ (or a 0-form symmetry $\mathbb{Z}_{5}$ ). These features are distinct from any known rank- $1 E_{n}$ theory, including the $E_{6}$ theory (which has $d_{H}=11$ ). Hence, we conjecture that the $\mathcal{T}_{\mathbf{X}}^{5 \mathrm{~d}}$ associated to the singularity (4.33) is a new rank-1 SCFT.

\subsection{One-form symmetries of the $4 \mathrm{~d}$ SCFTs $\mathrm{AD}\left[G, G^{\prime}\right]$}

In this subsection, we further illustrate our discussion of higher-form symmetries from section 2.7 , by computing the one-form symmetry $\mathfrak{f}$ (electric or magnetic) of numerous $4 \mathrm{~d}$ SCFTs of type $\operatorname{AD}\left[G, G^{\prime}\right]$. We simply need to compute the torsion subgroup $\mathfrak{h}_{2}=\mathfrak{f} \oplus \mathfrak{f} \subset$ $H_{2}\left(L_{5}(\mathbf{X}), \mathbb{Z}\right)$. 
For instance, for $\operatorname{AD}\left[A_{k}, A_{l}\right]$, we find that this group is always trivial. On the other hand, for $\left[A_{k}, D_{m}\right]$, we can have non-trivial torsion:

\begin{tabular}{|c|c|ccccccccccccc|}
\hline$\Gamma^{(1)}=\mathfrak{f}$ & $D_{4}$ & $D_{5}$ & $D_{6}$ & $D_{7}$ & $D_{8}$ & $D_{9}$ & $D_{10}$ & $D_{11}$ & $D_{12}$ & $D_{13}$ & $D_{14}$ & $D_{15}$ \\
\hline$A_{1}$ & 0 & 0 & 0 & 0 & 0 & 0 & 0 & 0 & 0 & 0 & 0 & 0 \\
$A_{2}$ & $\mathbb{Z}_{2}$ & 0 & 0 & $\mathbb{Z}_{2}$ & 0 & 0 & $\mathbb{Z}_{2}$ & 0 & 0 & $\mathbb{Z}_{2}$ & 0 & 0 \\
$A_{3}$ & 0 & $\mathbb{Z}_{2}$ & 0 & 0 & 0 & $\mathbb{Z}_{2}$ & 0 & 0 & 0 & $\mathbb{Z}_{2}$ & 0 & 0 \\
$A_{4}$ & 0 & 0 & $\mathbb{Z}_{2}^{2}$ & 0 & 0 & 0 & 0 & $\mathbb{Z}_{2}^{2}$ & 0 & 0 & 0 & 0 \\
$A_{5}$ & 0 & 0 & 0 & $\mathbb{Z}_{2}^{2}$ & 0 & 0 & 0 & 0 & 0 & $\mathbb{Z}_{2}^{2}$ & 0 & 0 \\
$A_{6}$ & 0 & 0 & 0 & 0 & $\mathbb{Z}_{2}^{3}$ & 0 & 0 & 0 & 0 & 0 & 0 & $\mathbb{Z}_{2}^{3}$ \\
$A_{7}$ & 0 & 0 & 0 & 0 & 0 & $\mathbb{Z}_{2}^{3}$ & 0 & 0 & 0 & 0 & 0 & 0 \\
$A_{8}$ & $\mathbb{Z}_{2}$ & 0 & 0 & $\mathbb{Z}_{2}$ & 0 & 0 & $\mathbb{Z}_{2}^{4}$ & 0 & 0 & $\mathbb{Z}_{2}$ & 0 & 0 \\
\hline
\end{tabular}

This includes the case $\left[A_{2}, D_{4}\right]$ studied above, which has $\mathfrak{f}=\mathbb{Z}_{2}$. Similarly, for $\left[D_{k}, D_{m}\right]$, we find:

\begin{tabular}{|c|c|cccccccccccc|}
\hline$\Gamma^{(1)}=\mathfrak{f}$ & $D_{4}$ & $D_{5}$ & $D_{6}$ & $D_{7}$ & $D_{8}$ & $D_{9}$ & $D_{10}$ & $D_{11}$ & $D_{12}$ & $D_{13}$ & $D_{14}$ & $D_{15}$ \\
\hline$D_{4}$ & 0 & 0 & 0 & $\mathbb{Z}_{2}$ & 0 & 0 & 0 & 0 & 0 & $\mathbb{Z}_{2}$ & 0 & 0 \\
$D_{5}$ & 0 & 0 & 0 & $\mathbb{Z}_{2}$ & 0 & $\mathbb{Z}_{2}^{2}$ & 0 & $\mathbb{Z}_{2}$ & 0 & 0 & 0 & $\mathbb{Z}_{2}$ \\
$D_{6}$ & 0 & 0 & 0 & 0 & 0 & 0 & 0 & $\mathbb{Z}_{2}^{2}$ & 0 & 0 & 0 & 0 \\
$D_{7}$ & $\mathbb{Z}_{2}$ & $\mathbb{Z}_{2}$ & 0 & 0 & 0 & $\mathbb{Z}_{2}$ & $\mathbb{Z}_{2}$ & 0 & 0 & $\mathbb{Z}_{2}^{3}$ & 0 & 0 \\
$D_{8}$ & 0 & 0 & 0 & 0 & 0 & 0 & 0 & 0 & 0 & 0 & 0 & $\mathbb{Z}_{2}^{3}$ \\
$D_{9}$ & 0 & $\mathbb{Z}_{2}^{2}$ & 0 & $\mathbb{Z}_{2}$ & 0 & 0 & 0 & $\mathbb{Z}_{2}$ & 0 & $\mathbb{Z}_{2}^{2}$ & 0 & $\mathbb{Z}_{2}$ \\
$D_{10}$ & 0 & 0 & 0 & $\mathbb{Z}_{2}$ & 0 & 0 & 0 & 0 & 0 & $\mathbb{Z}_{2}$ & 0 & 0 \\
\hline
\end{tabular}

As a last set of examples, consider the series:

$$
\left[E_{k}, A_{l}\right]: \begin{array}{|c|ccccccccccccccc|c|}
\hline \Gamma^{(1)}=\mathfrak{f} & A_{1} & A_{2} & A_{3} & A_{4} & A_{5} & A_{6} & A_{7} & A_{8} & A_{9} & A_{10} & A_{11} & A_{12} & A_{13} & A_{14} & A_{15} \\
\cline { 2 - 4 } & 0 & 0 & \mathbb{Z}_{3} & 0 & \mathbb{Z}_{2} & 0 & \mathbb{Z}_{3} & 0 & 0 & 0 & 0 & 0 & 0 & 0 & \mathbb{Z}_{3} \\
E_{7} & 0 & 0 & 0 & 0 & \mathbb{Z}_{3} & 0 & 0 & \mathbb{Z}_{2}^{3} & 0 & 0 & \mathbb{Z}_{3} & 0 & 0 & 0 & 0 \\
E_{8} & 0 & 0 & 0 & 0 & \mathbb{Z}_{5} & 0 & 0 & 0 & \mathbb{Z}_{3}^{2} & 0 & \mathbb{Z}_{5} & 0 & 0 & \mathbb{Z}_{2}^{4} & 0 \\
\hline
\end{array}
$$

These one-forms symmetries deserve further study. As a preliminary comment, we note that they always seem related to the existence of S-duality frames in which we have a weakly coupled gauge group. For instance, we have:

$$
\begin{aligned}
& {\left[A_{3}, E_{6}\right]: \quad \mathfrak{f}=\mathbb{Z}_{3}, \quad \Delta=\left\{\frac{5}{4}, \frac{5}{4}, \frac{3}{2}, \frac{3}{2}, \frac{3}{2}, 2, \frac{9}{4}, \frac{9}{4}, 3\right\},} \\
& {\left[A_{5}, E_{7}\right]: \quad \mathfrak{f}=\mathbb{Z}_{3}, \quad \Delta=\left\{\frac{5}{4}, \frac{5}{4}, \frac{3}{2}, \frac{3}{2}, \frac{3}{2}, \frac{7}{4}, 2,2, \frac{9}{4}, \frac{9}{4}, \frac{5}{2}, \frac{11}{4}, 3,3, \frac{7}{2}, \frac{15}{4}, \frac{9}{2}\right\},} \\
& {\left[A_{5}, E_{8}\right]: \quad \mathfrak{f}=\mathbb{Z}_{5}, \quad \Delta=\left\{\frac{7}{6}, \frac{4}{3}, \frac{4}{3}, \frac{3}{2}, \frac{3}{2}, \frac{5}{3}, \frac{5}{3}, 2, \frac{13}{6}, \frac{7}{3}, \frac{7}{3}, \frac{5}{2}, \frac{5}{2}, 3, \frac{19}{6}, \frac{10}{3}, \frac{10}{3}, 4, \frac{25}{6}, 5\right\},}
\end{aligned}
$$


These Coulomb-branch spectra are compatible with the existence of 'partially weaklycoupled description' with gauge group $\mathrm{SU}(3), \mathrm{SU}(3) \times \mathrm{SU}(3)$ and $\mathrm{SU}(5)$, respectively. This would explain the one-form symmetries if we could understand how strongly-coupled systems are coupled to these gauge groups, and why they preserve the center symmetry.

\section{Rank-zero 4d SCFTs from isolated toric singularities}

In the previous section, we discussed some isolated singularities that had $r=0$, and therefore correspond to rank-zero $5 \mathrm{~d}$ SCFTs $\mathcal{T}_{\mathbf{X}}^{5 \mathrm{~d}}$. Conversely, a rank-zero $4 \mathrm{~d}$ SCFT $\mathscr{T}_{\mathbf{X}}^{4 \mathrm{~d}}$ would arise from an isolated singularity such that $\widehat{r}=0$, as computed from the geometry. If we restrict ourselves to isolated hypersurface singularities, there is a unique canonical singularity that fits the bill

$$
x_{1}^{2}+x_{2}^{2}+x_{3}^{2}+x_{4}^{2}=0,
$$

the conifold singularity, which engineers the free hypermultiplet in Type IIB string theory. To obtain potentially more interesting rank-zero 4d SCFTs, we need to consider more general canonical singularities. In that case, we would also need to properly define what we mean by the quantities $f$ and $\widehat{r}$ geometrically, for the deformed singularity. A natural conjecture is that there always exists a mixed Hodge structure on the deformation space of any $\mathbf{X}$, in some appropriate sense, such that:

$$
f=\operatorname{dim} H^{2,2}(\widehat{\mathbf{X}}), \quad \widehat{r}=\operatorname{dim} H^{1,2}(\widehat{\mathbf{X}})=\operatorname{dim} H^{2,1}(\widehat{\mathbf{X}}),
$$

generalizing the hypersurface case. This is bound to be somewhat more subtle because, in general, the space of deformations can have several distinct branches, which can intersect in a non-trivial manner. In any case, $\widehat{r}=0$ would correspond to a threefold whose deformation parameters all have a scaling dimension $\Delta=1$, once we identify the correct $\mathrm{U}(1)_{r}$ scaling action on the coordinate ring of the singularity. In the following, we make some preliminary comments on the case, when $\mathbf{X}$ is a toric singularity.

\subsection{Toric singularities and deformations}

An isolated toric Calabi-Yau threefold singularity $\mathbf{X}$ corresponds to a strictly convex toric diagram. ${ }^{22}$ The basic data of any of its crepant resolutions, $\widetilde{\mathbf{X}}$, is read off from the toric diagram as:

$$
f=n_{E}-3, \quad r=n_{I},
$$

where $n_{E}$ and $n_{I}$ denote the number of external and internal points, respectively, in the toric diagram of $\mathbf{X}$. Here, $f$ again denotes the number of compact 2-cycles in $\widetilde{\mathbf{X}}$ dual to non-compact divisors, and it therefore counts the number of flavor background vector multiplet on the Coulomb branch of $\mathcal{T}_{\mathbf{X}}^{5 \mathrm{~d}}$. On the other hand, $\widehat{d}_{H}=r+f$, the dimension of the Kähler cone, must still be the dimension of the Higgs branch of $\mathscr{T}_{\mathbf{X}}^{4 \mathrm{~d}}$.

The $5 \mathrm{~d}$ SCFT $\mathcal{T}_{\mathbf{X}}^{5 \mathrm{~d}}$ from an isolated toric singularity has a 1-form (or a magnetic 2-form) symmetry [80, 81], which arise from the geometry of the boundary five-manifold $L_{5}(\mathbf{X})$,

\footnotetext{
${ }^{22}$ For recent work in the $5 \mathrm{~d}$ context and a summary of toric geometry see e.g. [15, 27].
} 
which does not need to be simply-connected (unlike in the hypersurface case). This then implies that the putative $4 \mathrm{~d}$ SCFT from IIB on $\mathbf{X}$ has a 0 -form or 2-form symmetry given by the same discrete group at the one-form symmetry in $5 \mathrm{~d}$, namely Tor $H_{1}\left(L_{5}(\mathbf{X}), \mathbb{Z}\right)$. This torsion group is readily computed from the toric data [80, 81].

The versal space of deformations of any isolated toric singularity has dimension [154]:

$$
\widetilde{d}_{H}=\#(\text { versal deformations }) \leq f
$$

More precisely, there are always exactly $f$ first-order deformations, but obstructions generally arise at higher order. ${ }^{23}$ There also exists an explicit algorithm to construct the deformed coordinate ring, for any deformation [154].

Let us assume that $\widehat{d}_{H}=f$ for simplicity. Then, the versal deformation space has a single branch, which is the case most similar to the hypersurface singularities that we studied so far. A generic versal deformation of $\mathbf{X}$ then leads to a deformed singularity $\widehat{\mathbf{X}}$ with $f$ 3-cycles. All these 3 -cycles can undergo geometric transitions to the $N$ 'flavor' 2-cycles in the (partially) resolved geometry. Thus, even without a detailed analysis of the $\mathrm{U}(1)_{r}$ action on the singularity, we see that all these deformations must correspond to mass term in the putative $4 \mathrm{~d}$ SCFT $\mathscr{T}_{\mathbf{X}}^{4 \mathrm{~d}}$ engineered in IIB. Therefore, the $5 \mathrm{~d} / 4 \mathrm{~d}$ correspondence studied in this paper directly suggests that toric singularities geometrically engineer rankzero $4 d S C F T s$ in Type IIB. This was first proposed in [46] for toric orbifolds. Here, we will present one interesting class of example with $\widetilde{d}_{H}=f=1$, and present evidence that such theories $\mathscr{T}_{\mathbf{X}}^{4 \mathrm{~d}}$ can be non-trivial, in a limited sense that their Higgs branch can be a non-trivial hyper-Kähler cone. A more detailed analysis is left for future work.

\subsection{The $Y^{N, 0}$ geometry, $5 \mathrm{~d} \mathrm{SU}(N)_{0}$ and rank-zero $4 \mathrm{~d}$ SCFTs}

Consider the toric diagram, which is a lattice polygon with four vertices at position $(0,0)$, $(1,0),(1, N)$ and $(2, N)$

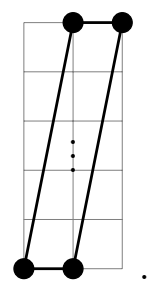

Note that we have $f=1$ and $r=N-1$. This toric singularity is known as the cone over $Y^{N, 0}$ [155]. It engineers a rank $N-15 \mathrm{~d}$ SCFT with a single deformation, which triggers a flow to the $5 \mathrm{~d}$ gauge theory $\mathrm{SU}(N)_{0}$. In the limiting case $N=1$, we have the conifold singularity (which can also be viewed as an 'SU(1)' theory in $5 \mathrm{~d}$ [15]), and for $N=2$ we have the complex cone over $\mathbb{F}_{0}$, engineering the rank-one $E_{1}$ SCFT. The space of versal deformations (5.5) is one dimensional, giving us a smooth local Calabi-Yau threefold $\widetilde{\mathbf{X}}$. For instance, the $E_{1}$ singularity is a non-complete intersection in $\mathbb{C}^{9}$, which admits single

\footnotetext{
${ }^{23}$ Hypersurfaces are 'too simple' in that particular regard, since there is no obstruction in that case.
} 
a consistent deformation to [154]:

$$
\begin{array}{llll}
x_{1} x_{3}=\left(x_{9}-\varepsilon\right)^{2}, & x_{2} x_{4}=x_{9}^{2}, & x_{5} x_{7}=\left(x_{9}-\varepsilon\right) x_{9}, & x_{6} x_{8}=\left(x_{9}-\varepsilon\right) x_{9}, \\
x_{5} x_{8}=x_{1} x_{9}, & x_{5} x_{6}=x_{2}\left(x_{9}-\varepsilon\right), & x_{6} x_{7}=x_{3} x_{9}, & x_{7} x_{8}=x_{4}\left(x_{9}-\varepsilon\right), \\
x_{1} x_{6}=x_{5}\left(x_{9}-\varepsilon\right), & x_{2} x_{8}=x_{5} x_{9}, & x_{2} x_{7}=x_{6} x_{9}, & x_{3} x_{5}=x_{6}\left(x_{9}-\varepsilon\right), \\
x_{3} x_{8}=x_{7}\left(x_{9}-\varepsilon\right), & x_{4} x_{6}=x_{7} x_{9}, & x_{1} x_{7}=x_{8}\left(x_{9}-\varepsilon\right), & x_{4} x_{5}=x_{8} x_{9},
\end{array}
$$

with the isolated singularity at $\varepsilon=0$.

The Higgs branch of $\mathcal{T}_{\mathbf{X}}^{5 \mathrm{~d}}$ can be studied using the tropical-geometric methods developed in [78], which are easily specialized to the case of an isolated toric geometry. We summarize the relevant algorithm in appendix B. For the toric singularity (5.5), one finds the following magnetic quiver for $\mathcal{T}_{\mathbf{X}}^{5 \mathrm{~d}}$ :

$$
\mathrm{MQ}^{(5)}=\stackrel{1}{\bullet} \boldsymbol{\bullet}^{-1}
$$

modulo the overall $\mathrm{U}(1)$. Equivalently, $\mathrm{MQ}^{(5)}=\mathrm{SQED}\left[N_{f}=N\right]$. Its Coulomb branch gives the $5 \mathrm{~d}$ Higgs branch, which is the $A_{N-1}$ Kleinian singularity:

$$
\mathcal{M}_{H}^{5 \mathrm{~d}}=\mathrm{CB}\left[\mathrm{MQ}^{(5)}\right]=\mathbb{C}^{2} / \mathbb{Z}_{N}
$$

The $3 \mathrm{~d}$ mirror theory is the $\widehat{a}_{N-1}$ affine quiver:

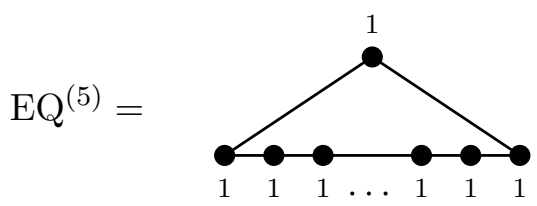

This quiver has a gauge group $\mathrm{U}(1)^{N} / \mathrm{U}(1)$ and a single flavor symmetry, the 'baryonic symmetry' $\mathrm{U}(1)_{B}$, which assigns the same charge, $B=b_{0}$, to all the hypermultiplets in the loop. According to our general prescription, to obtain the magnetic quiver of the $4 \mathrm{~d}$ SCFT $\mathscr{T}_{\mathbf{X}}^{4 \mathrm{~d}}$, we should gauge this symmetry:

$$
\mathrm{MQ}^{(4)}=\mathrm{EQ}^{(5)} / \mathrm{U}(1)_{B}
$$

There are two natural choices for the normalization of $\mathrm{U}(1)_{B}$. The $\mathrm{CB}$ of $(5.7)$ is described as $T^{+} T^{-}=\Phi^{N}$, where $\Phi$ is the vector multiplet scalar of $\operatorname{SQED}\left[N_{f}=N\right]$, and $T^{ \pm}$are its monopole operators [156] of topological charge $B= \pm 1$. The topological symmetry is identified with the baryonic symmetry in the mirror (5.9), whose Higgs branch is described as $\mathcal{B}^{+} \mathcal{B}^{-}=M^{N}$, with $\mathcal{B}^{+}=H_{1} \cdots H_{N}$ schematically, where $H_{i}, i=1, \cdots, N$ are the hypermultiplets of the circular quiver (5.9). Thus, we have the two possible charge assignments:

$$
\begin{array}{lllll}
B\left[T^{ \pm}\right]= \pm 1 & \text { in } \mathrm{MQ}^{(5)} & \leftrightarrow & B\left[H_{i}\right]=\frac{1}{N} & \text { in } \mathrm{EQ}^{(5)}, \\
B\left[T^{ \pm}\right]= \pm N & \text { in } \mathrm{MQ}^{(5)} & \leftrightarrow & B\left[H_{i}\right]=1 & \text { in } \mathrm{EQ}^{(5)} .
\end{array}
$$


The magnetic quiver of the putative $4 \mathrm{~d}$ SCFT is then given by a $\mathrm{U}(1)^{N}$ gauge theory with $N$ hypermultiplets and the charges:

\begin{tabular}{c|c|cccccc|}
\hline & $H_{1}$ & $H_{2} \cdots$ & $H_{N-2}$ & & $H_{N-1}$ & $H_{N}$ \\
\hline $\mathrm{U}(1)_{1}$ & 1 & 0 & $\cdots$ & 0 & 0 & -1 \\
$\mathrm{U}(1)_{2}$ & -1 & 1 & $\cdots$ & 0 & 0 & 0 \\
$\vdots$ & $\vdots$ & & $\ddots$ & & & $\vdots$ \\
$\mathrm{U}(1)_{N-1}$ & 0 & 0 & $\cdots$ & -1 & 1 & 0 \\
$\mathrm{U}(1)_{B}$ & $b_{0}$ & $b_{0}$ & $\cdots$ & $b_{0}$ & $b_{0}$ & $b_{0}$ \\
\hline
\end{tabular}

Here, $b_{0}=B\left[H_{i}\right]$ in either normalization in (5.11). The mirror of (5.12), which is the electric quiverine of $\mathscr{T}_{\mathbf{X}}^{4 \mathrm{~d}}$, is obtained by gauging the topological symmetry of $\mathrm{MQ}^{(5)}$. Using the results of [75], it is easy to see that, depending on the normalization (5.11), we obtain:

(i) $\quad \mathrm{EQ}^{(4)}=(N$ hypermultiplets $)$,

(ii) $\quad \mathrm{EQ}^{(4)}=(N$ hypermultiplets $) / \mathbb{Z}_{N}$.

In the first case, we have $N$ free hypermultiplets, while in the second case we have a $\mathbb{Z}_{N}$ gauge theory of $N$ hypermultiplets. This discrete gauging leads to a non-trivial Higgs branch chiral ring, as can be ascertained by computing the HB Hilbert series. This is most easily done by computing the Hilbert series of the $\mathrm{CB}$ of $\mathrm{MQ}^{(4)}$, as discussed in appendix A.2. Of course, in the first case, we have the Hilbert series of $\mathbb{C}^{2 N}$, while for the $\mathbb{Z}_{N}$ gauge theory we find:

$$
\begin{aligned}
\mathrm{HS}_{\mathcal{M}_{H}^{4 \mathrm{~d}}}^{(N=2)} & =\frac{1+6 t^{2}+t^{4}}{\left(1-t^{2}\right)^{4}} \\
\mathrm{HS}_{\mathcal{M}_{H}^{4 d}}^{(N=3)} & =\frac{1-3 t+12 t^{2}-11 t^{3}+12 t^{4}-3 t^{5}+t^{6}}{(1-t)^{6}\left(1+t+t^{2}\right)^{3}} \\
\mathrm{HS}_{\mathcal{M}_{H}^{4 \mathrm{~d}}}^{(N=4)} & =\frac{1+12 t^{2}+108 t^{4}+212 t^{6}+358 t^{8}+212 t^{10}+108 t^{12}+12 t^{14}+t^{16}}{\left(1-t^{2}\right)^{8}\left(1+t^{2}\right)^{4}},
\end{aligned}
$$

etc. For $N=2$, this corresponds to the minimal nilpotent orbit of $\operatorname{Sp}(2)$, while for $N>2$ we have a Higgs branch with a $\mathrm{U}(N)$ flavor group. From the mirror description (5.13), we know that these Higgs branches are $\mathbb{Z}_{N}$ quotients of $\mathbb{C}^{2 N}$ preserving the hyper-Kähler structure - that is, quotients preserving $3 \mathrm{~d} \mathcal{N}=4$ supersymmetry. In the $N=2$ case, we also know from the analysis of [111] that the central charges $a$ and $c$ are the same as for two free hypermultiplets, yet the $4 \mathrm{~d}$ theory has a non-trivial Higgs-branch chiral ring.

Let us also note that the $5 \mathrm{~d} \operatorname{SCFT} \mathcal{T}_{\mathbf{X}}^{5 \mathrm{~d}}$ has a $\mathbb{Z}_{N} 1$-form symmetry or a 2 -form symmetry, corresponding to having a gauge theory $\mathrm{SU}(N)_{0}$ or $\operatorname{PSU}(N)_{0}$ in the IR description [80]. This choice parallels the choice of global structure of $\mathscr{T}_{\mathbf{X}}^{4 \mathrm{~d}}$ apparent in (5.11), in which case $\mathscr{T}_{\mathbf{X}}^{4 \mathrm{~d}}$ should have a 0 -form symmetry or a 2 -form symmetry, respectively. Finally, it would be tempting to trivially 'uplift' the $3 \mathrm{~d} \mathcal{N}=4$ theory (5.13) to 'conclude' that the $4 \mathrm{~d} \mathcal{N}=2$ SCFT $\mathscr{T}_{\mathbf{X}}^{4 \mathrm{~d}}$ consists of $N$ free hypermultiplets, or a discrete gauging thereof (which then has a two-form symmetry). Of course, this is not guaranteed, since the flow from $4 \mathrm{~d}$ to $3 \mathrm{~d}$ 
could wash out some interesting information. It would certainly be interesting to explore these subtle issues further.

\section{Acknowledgments}

We thank Fabio Apruzzi, Marieke van Beest, Lakshya Bhardwaj, Antoine Bourget, Stefano Cremonesi, Michele del Zotto, Julius Eckhard, Simone Giacomelli, Amihay Hanany, Zohar Komargodski, Horia Magureanu, Mario Martone, James Sparks, Yuji Tachikawa and Yifan Wang for very interesting discussions and comments on the draft. CC also gratefully acknowledges Yvette Siegert for her terminological contribution. CC is a Royal Society University Research Fellow and a Research Fellow at St John's College, Oxford. The work of SSN and YW is supported by the ERC Consolidator Grant number 682608 "Higgs bundles: Supersymmetric Gauge Theories and Geometry (HIGGSBNDL)". SSN acknowledges support also from the Simons Foundation.

\section{A Higgs-branch Hilbert series from magnetic quivers}

In this appendix, we present some relevant details on some of the Hilbert series computations mentioned in the main text. For completeness, let us first review some well-known technology. We would like to consider the Higgs branch in an SCFT with eight supercharges:

$$
\mathcal{M}_{H}=\operatorname{Spec} \mathcal{R}_{H}
$$

Here, $\mathcal{R}_{H}$ denote the $\mathrm{HB}$ chiral ring, which is graded by the $\mathrm{SU}(2)_{R}$ charge $R \in \frac{1}{2} \mathbb{Z}$, and the moduli space must be a hyper-Kähler singularity. The Hilbert series of $\mathcal{M}_{H}$ is the formal series over the chiral ring operators weighted by their $R$-charges:

$$
\operatorname{HS}_{\mathcal{M}_{H}}(t)=\sum_{\mathcal{O} \in \mathcal{R}_{\mathrm{H}}} t^{2 R[\mathcal{O}]}
$$

Given the Hilbert series, we have some simple 'diagnostic tests' of the structure of the Higgs branch (see [157] for a nice review):

- The quaternionic dimension of $\mathcal{M}_{H}$ corresponds to the pole at $t=1$ :

$$
\operatorname{HS}_{\mathcal{M}_{H}} \sim \frac{1}{(1-t)^{2 \operatorname{dim}\left(\mathcal{M}_{H}\right)}}
$$

- Expanding around $t=0$, the order- $t$ term gives the number $n_{\text {free }}$ of free hypermultiplets $\left(\mathbb{C}^{2}\right.$ factors in $\left.\mathcal{M}_{H}\right)$ while the order- $t^{2}$ term gives the number $n_{\mathrm{cc}}$ of conserved currents:

$$
\mathrm{HS}_{\mathcal{M}_{H}}(t)=1+2 n_{\text {free }} t+n_{\mathrm{cc}} t^{2}+\cdots
$$

Each free hypermultiplet corresponds to $\mathrm{HS}_{\mathbb{C}^{2}}=(1-t)^{-2}$, which factorizes from the full Hilbert series. 
- By taking the plethystic logarithm ${ }^{24} \mathrm{PL}\left(\mathrm{HS}_{\mathcal{M}_{H}}(t)\right)$, we can find the number of generators $n_{H}$ of $\mathcal{R}_{H}$ (with their $R$-charges), and the number of relations amongst them. The PL terminates if and only if $\mathcal{M}_{H}$ is a complete intersection in $\mathbb{C}^{n_{H}}$. In particular, $\mathrm{PL}=2 t$ for a free hypermultiplet.

One can refine this analysis by including fugacities for various flavor symmetries; in this paper, we only considered the 'unrefined' HS, for simplicity.

For $3 \mathrm{~d} \mathcal{N}=4$ theories, we can also compute the HS of the Coulomb branch, which is itself hyper-Kähler, with the $\mathrm{CB}$ operators weighted by the $\mathrm{SU}(2)_{C} R$-charge. Indeed, we would like to understand the $3 \mathrm{~d} \mathcal{N}=4$ Coulomb branches of the 'magnetic quivers' of $4 \mathrm{~d}$ or 5d SCFTs. Assuming the magnetic quiver is given by an explicit gauge-theory UV description in $3 \mathrm{~d}$, with gauge group $G$, its CB Hilbert series is easily computed thanks to the monopole formula [49]:

$$
\mathrm{HS}_{\mathrm{CB}}(t)=\sum_{\mathfrak{m} \in \Gamma / W_{G}} t^{2 R(\mathfrak{m})} P_{G}(t ; \mathfrak{m}), \quad P_{G}(t ; \mathfrak{m})=\prod_{k=1}^{\operatorname{rank}(G)} \frac{1}{1-t^{d_{k}\left(G_{\mathfrak{m}}\right)}},
$$

where the sum is over the GNO-quantized magnetic fluxes of $G$ (modulo the Weyl group), and $P_{G}(t ; \mathfrak{m})$ denotes the Coulomb-branch index of the gauge group $G_{\mathfrak{m}}$ that commutes with the flux $\mathfrak{m}$ - that is, $d_{k}\left(G_{\mathfrak{m}}\right)$ are the dimension of the Casimir invariants of $G_{\mathfrak{m}}$. (For instance, we have $P_{\mathrm{U}(N)}(t ; 0)=\prod_{k=1}^{N}\left(1-t^{k}\right)^{-1}$.) Finally, $R(\mathfrak{m})$ is the quantum dimension of the monopole operator for this magnetic flux [147]:

$$
R(\mathfrak{m})=-\sum_{\alpha \in \Delta^{+}}|\alpha(\mathfrak{m})|+\frac{1}{2} \sum_{\rho \in \mathfrak{R}}|\rho(\mathfrak{m})|,
$$

where $\Delta^{+}$is the set of positive roots of $\mathfrak{g}=\operatorname{Lie}(G)$, and the second sum runs over all the weights of the (generally reducible) representation $\mathfrak{R}$ of $\mathfrak{g}$ under which the hypermultiplets transform. Note that the monopole formulas 'knows' about the global structure of $G$ through the sum over the magnetic flux lattices. ${ }^{25}$

Let us also note that, for abelian theories with gauge group $G=\prod_{k=1}^{r} \mathrm{U}(1)_{k}$, the monopole formula (A.5) simplifies to:

$$
\operatorname{HS}_{\mathrm{CB}}(t)=\frac{1}{\left(1-t^{2}\right)^{r}} \sum_{\mathfrak{m} \in \mathbb{Z}^{r}} t^{2 R(\mathfrak{m})}, \quad R(\mathfrak{m})=\sum_{i} \sum_{k=1}^{r}\left|q_{i}^{k} \mathfrak{m}_{k}\right|
$$

where the sum $\sum_{i}$ runs over all the hypermultiplets $H_{i}$, with electric charges $q_{i}^{k}$.

\footnotetext{
${ }^{24}$ Recall the definition of the plethystic exponentional (giving us the 'multi-particle states' from the 'single-particle states' [158]), and its inverse, the plethystic logarithm (PL). For a single variable $t$, we have:

$$
\mathrm{PE}[f(t)]=\exp \left(\sum_{p=1}^{\infty} \frac{1}{p} f\left(t^{p}\right)\right), \quad \mathrm{PL}[g(t)]=\exp \left(\sum_{k=1}^{\infty} \frac{\mu(k)}{k} \log g\left(t^{k}\right)\right),
$$

assuming $f(0)=0$ and $g(0)=1$; here, $\mu(k)$ is the Möbius function.

${ }^{25}$ Interestingly, the HS can also be viewed a supersymmetric partition function - under some assumptions, it is both a twisted partition function on $S^{2} \times S^{1}[159]$ and a limit of the 3d superconformal index [160].
} 


\section{A.1 The $\mathrm{CB}$ of $\mathrm{EQ}^{(4)}$ for $\mathrm{AD}\left[A_{2}, D_{4}\right]$}

Consider the $\mathrm{CB}$ of the $3 \mathrm{~d}$ quiver of eq. (4.31), with either $\mathrm{SU}(2)$ or $\mathrm{SO}(3)$ as the central node. In the $\mathrm{SU}(2)$ case, the HS takes the form:

$$
\mathrm{HS}_{\mathrm{MQ}^{(5)}\left[\mathbf{X}_{\left[A_{2}, D_{4}\right]}\right]}(t)=\sum_{\mathfrak{m} \in \mathbb{Z}^{3}} \frac{1}{\left(1-t^{2}\right)^{3}}\left(\frac{t^{2 R(\mathfrak{m}, 0)}}{1-t^{4}}+\sum_{\mathfrak{n}>0} \frac{t^{2 R(\mathfrak{m}, \mathfrak{n})}}{1-t^{2}}\right)
$$

with $\mathfrak{m}=\left(\mathfrak{m}_{1}, \mathfrak{m}_{2}, \mathfrak{m}_{3}\right)$ for the three $\mathrm{U}(1)$ factors, and $\mathfrak{n} \in \mathbb{Z}_{>0}$ the $\mathrm{SU}(2)$ fluxes, and:

$$
R(\mathfrak{m}, \mathfrak{n})=-|2 \mathfrak{n}|+\frac{1}{2} \sum_{i=1}^{3}\left(\left|\mathfrak{n}+\mathfrak{m}_{i}\right|+\left|-\mathfrak{n}+\mathfrak{m}_{i}\right|\right)
$$

we can write this as:

$$
\mathrm{HS}_{\mathrm{MQ}^{(5)}\left[\mathbf{X}_{\left[A_{2}, D_{4}\right]}(\mathrm{SU}(2))\right.}(t)=\frac{\mathrm{h}(t ; 0)^{3}}{1-t^{4}}+\sum_{\mathfrak{n}=1}^{\infty} \frac{t^{-4 \mathfrak{n}} \mathrm{h}(t ; \mathfrak{n})^{3}}{1-t^{2}}
$$

corresponding to the $\mathrm{SU}(2)$ gauging of three copies of $\mathrm{SQED}\left[N_{f}=2\right]$, whose CB Hilbert series, with background flux $\mathfrak{n}$ for the $\mathrm{SU}(2)$ flavor group, can be easily computed:

$$
\mathrm{h}(t ; \mathfrak{n}) \equiv \operatorname{HS}_{\mathrm{CB}\left[\operatorname{SQED}\left[N_{f}=2\right]\right]}(t ; \mathfrak{n})=\frac{t^{2|\mathfrak{n}|}\left(1+t^{2}+2|\mathfrak{n}|\left(1-t^{2}\right)\right)}{\left(1-t^{2}\right)^{2}}
$$

This gives:

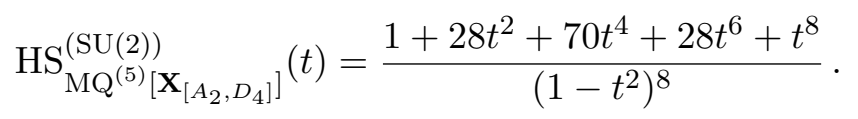

Similarly, the $\mathrm{SO}(3)$ gauging corresponds to summing over $\mathfrak{n} \in \frac{1}{2} \mathbb{Z}_{>0}$ in (A.8), and one finds:

$$
\mathrm{HS}_{\mathrm{MQ}^{(5)}\left[\mathbf{X}_{\left[A_{2}, D_{4}\right]}(\mathrm{SO}(3))\right.}(t)=\frac{1}{(1-t)^{8}} .
$$

\section{A.2 Gauging the affine $\widehat{a}_{N-1}$ quiver}

Consider the $\mathrm{CB}$ Hilbert series of the $\mathrm{MQ}^{(4)}$ (5.12). The monopole formula gives:

$$
\mathrm{HS}=\frac{1}{(1-t)^{N}} \sum_{\mathfrak{m}_{B} \in \mathbb{Z}} \sum_{\mathfrak{m}_{1} \in \mathbb{Z}} \cdots \sum_{m_{N-1} \in \mathbb{Z}} t^{2 R\left(\mathfrak{m}_{B}, \mathfrak{m}\right)},
$$

with $2 R\left(\mathfrak{m}_{B}, \mathfrak{m}\right)=\sum_{i=1}^{N-1}\left|m_{i}-m_{i+1}+b_{0} \mathfrak{m}_{B}\right|$. For $b_{0}=\frac{1}{N}$, we can perform a $\operatorname{SL}(N, \mathbb{Z})$ transformation on the electric charges, so that we have $N$ copies of $\operatorname{SQED}\left[N_{f}=1\right]$, mirror to $N$ free hypers. In general, we can change basis so that:

$$
\mathrm{HS}=\frac{1}{(1-t)} \sum_{\mathfrak{m}_{B} \in \mathbb{Z}}\left[\frac{1}{(1-t)^{N-1}} \sum_{\mathfrak{n}_{1} \in \mathbb{Z}} \cdots \sum_{n_{N} \in \mathbb{Z}} t^{2 R\left(\mathfrak{m}_{B}, \mathfrak{n}\right)}\right],
$$

with:

$$
2 R\left(\mathfrak{m}_{B}, \mathfrak{n}\right)=\left|b_{0} N \mathfrak{m}_{B}-\sum_{i=1}^{N-1} \mathfrak{n}_{i}\right|+\sum_{i=1}^{N-1}\left|\mathfrak{n}_{i}\right|
$$


We can first fix $\mathfrak{m}_{B}$ and do the sum over $\mathfrak{n}_{i}$ in (A.15), which gives the HS of the CB of the $\widehat{a}_{N-1}$ quiver, corresponding to the minimal nilpotent orbit of $a_{N-1}$, in the presence of background magnetic flux $\mathfrak{m}_{B}$. For instance:

$$
\begin{aligned}
& N=2: \quad \operatorname{HS}\left(\mathfrak{m}_{\mathrm{B}}\right)=\frac{t^{2 b_{0} \mathfrak{m}_{B}}\left(1+t^{2}-2 b_{0} \mathfrak{m}_{B}\left(1-t^{2}\right)\right)}{\left(-1+t^{2}\right)^{2}} \\
& N=3: \quad \operatorname{HS}\left(\mathfrak{m}_{\mathrm{B}}\right)=\frac{t^{3 b_{0} \mathfrak{m}_{B}}\left(9 b_{0}^{2} \mathfrak{m}_{B}^{2}\left(1-t^{2}\right)^{2}-9 b_{0} \mathfrak{m}_{B}\left(1-t^{4}\right)+2\left(1+4 t^{2}+t^{4}\right)\right)}{2\left(1-t^{2}\right)^{4}}
\end{aligned}
$$

etc. Then, summing over $\mathfrak{m}_{B}$, with either choice of $b_{0}$, we obtain the result shown in (5.14). Note that we can view this $\mathrm{CB}$ (for this $\mathrm{MQ}^{(4)}$ ) as a $\mathbb{C}^{2}$ fibration over the minimal nilpotent orbit of $a_{N-1}$ (the $\mathrm{CB}$ of the 'ungauged' theory, $\mathrm{EQ}^{(5)}$ ), as this computation makes rather explicit.

\section{B Magnetic quivers $\mathrm{MQ}^{(5)}$ for isolated toric singularities}

In this appendix we summarize the construction of the magnetic quiver starting with the toric diagram [78]. Here we will limit ourselves to the strictly convex toric geometries, as these are relevant in the context of the $4 \mathrm{~d}$ SCFTs.

Consider then a strictly convex lattice polygons $P$ in $\mathbb{Z}^{2} \subset \mathbb{R}^{2}$, with vertices $\boldsymbol{v}_{i}, i=$ $1, \cdots, n$. The flavor rank is $f=n-3$ and the strict convexity requires that there are no lattice points along the edges connecting two of the vertices. The proposal in [78] is that the magnetic quiver is obtained from a Minkowski sum decomposition of the polygon into convex polygons $P_{i}$

$$
P=P_{1}+\cdots+P_{c},
$$

where $A+B=\{a+b ; a \in A, b \in B\}$ is the Minkowski sum of two convex polygons of dimension 1 or 2 (i.e. edge segments or 2 d polygons). This induces an edge coloring, by coloring each edge, that arises from $P_{i}$ into one color. An example is the toric polygon for the $5 \mathrm{~d} E_{1}$ theory, which has an IR description in $5 \mathrm{~d}$ as $\mathrm{SU}(2)_{0}$

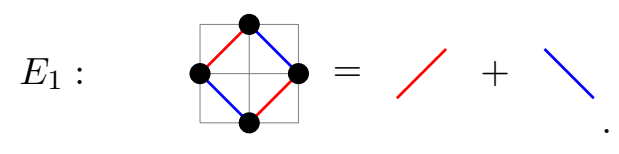

The magnetic quiver for these isolated toric models is computed as follows:

1. Determine all distinct Minkowski sum decompositions of $P$, and associated edge colorings.

2. Each edge coloring can be extended by adding internal edges, such that the polygon is covered by polygons that have a single edge color, or by parallelograms, which are at most bi-colored, with opposite edges of the same color.

3. Each color gets associated a node in the magnetic quiver of multiplicity 1 . 
4. The number of edges $k_{c_{1}, c_{2}}$ between nodes associated to $c_{1}$ and $c_{2}$ are determined by the mixed volume, i.e.

$$
k_{c_{1}, c_{2}}=\operatorname{Area}\left(G_{c_{1}, c_{2}}\right)=\text { area of the } c_{1}, c_{2} \text { bicolored paralellogram } .
$$

For the $E_{1}$ there is one Minkowski sum decomposition, shown already above, from which the magnetic quiver follows to be two vertices and one double-line between them

$$
\operatorname{MQ}\left(E_{1}\right)=\stackrel{1}{\rightleftarrows}
$$

We will now focus on examples where the deformation theory is unobstructed, and therefore the Higgs branch dimension agrees with the flavor rank $f$. The $E_{3}$-theory (in $5 \mathrm{~d}$ the rank 1 theory with $\mathrm{SU}(2)+2 \boldsymbol{F}$ IR description) is of this type:

$$
E_{3}: \bigcap_{-1}^{\infty}=-1+/=\nabla+\Delta
$$

This implies the magnetic quiver, which has two components

$$
\mathrm{MQ}\left(E_{3}\right)=\stackrel{1}{\longrightarrow}
$$

An infinite class of theories is the set of toric singularities with vertices

$$
\{(0,0),(1,0),(1, N),(2, N)\}
$$

i.e.

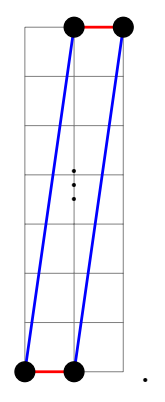

This toric diagram gives rise to the SCFT with IR description $\mathrm{SU}(N)_{0}$ in M-theory. Here we will consider it in Type IIB. As the coloring indicates, there is precisely one magnetic quiver, which has two multiiplicity 1 nodes connected by $N$ edges, i.e. the $A_{N-1}$ Kleinian singularity

$$
A_{N-1}=\stackrel{1}{\bullet} \quad{ }^{N} \stackrel{1}{\bullet}
$$




\section{Rank- $N E_{1}$ magnetic quivers from the $(p, q)$-webs}

In this appendix, we discuss the brane-web construction of the magnetic quiver $\mathrm{MQ}^{(5)}$ for the rank $N E$-strings, focusing on the AS matter representation. The brane-webs for the rank $N E_{n}$-strings were determined in $[14,121]$ and a derivation for their entire decoupling tree will appear in [161]. Here, our interest is in the AS hypermultiplet phase transition, that we discussed in section 3.1. To illustrate this point, we simply consider the case with no fundamental flavor, i.e. the theory with an IR description:

$$
\operatorname{Sp}(N)+1 \boldsymbol{A} \boldsymbol{S}
$$

Its UV fixed point is the rank- $N E_{1}$ theory. We present the generalized toric diagram for both the theory with the massless and massive anti-symmetric. Consider for instance the $N=2 E_{0}$-theory, which has generalized toric diagram [78]

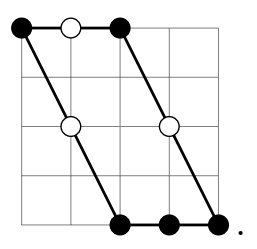

In the dual brane-web the white dots indicate that the associated 5-branes dual to the edge segments adjacent to it end on the same 7-brane - for a review, we refer the reader to [121]. The magnetic quiver of this theory is readily computed using the methods in [78]. For general $N$, one finds:

$$
\mathrm{MQ}^{(5)}=\stackrel{1}{\bullet} \stackrel{N}{\stackrel{N}{\longrightarrow}} \stackrel{N}{\bullet},
$$

This is $N$ times the affine $\mathfrak{a}_{1}$ Dynkin diagram, with an additional multiplicity- 1 node.

We can mimick the transition described in section 3.1 at the level of the brane web. This is realized by the model where the 5-branes, which realize the $\boldsymbol{A S}$, end on one 7-brane, which effectively corresponds to decoupling of $N-15$-brane segments in the transverse direction. For $N=2$, for instance, we then obtain:

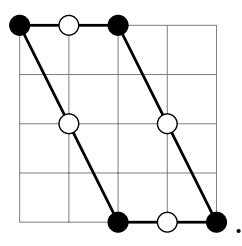

Here and for general $N$, the $\mathrm{MQ}^{(5)}$ is then simply $N$ times the affine $\mathfrak{a}_{1}$ Dynkin diagram, in agreement with our discussion in section 3.1: ending $N$ 5-branes on the same 7 -brane, and displacing $N-1$ 5-brane segments transversely, corresponds to the 'partial Higgs phase' on the $\mathrm{CB}$ of the $5 \mathrm{~d}$ gauge theory.

Open Access. This article is distributed under the terms of the Creative Commons Attribution License (CC-BY 4.0), which permits any use, distribution and reproduction in any medium, provided the original author(s) and source are credited. 


\section{References}

[1] E. Witten, Phase transitions in M-theory and F-theory, Nucl. Phys. B 471 (1996) 195 [hep-th/9603150] [INSPIRE].

[2] S.H. Katz, A. Klemm and C. Vafa, Geometric engineering of quantum field theories, Nucl. Phys. B 497 (1997) 173 [hep-th/9609239] [InSPIRE].

[3] A. Klemm, W. Lerche, P. Mayr, C. Vafa and N.P. Warner, Selfdual strings and $N=2$ supersymmetric field theory, Nucl. Phys. B 477 (1996) 746 [hep-th/9604034] [INSPIRE].

[4] M. Bershadsky, K.A. Intriligator, S. Kachru, D.R. Morrison, V. Sadov and C. Vafa, Geometric singularities and enhanced gauge symmetries, Nucl. Phys. B 481 (1996) 215 [hep-th/9605200] [INSPIRE].

[5] N. Seiberg, Five-dimensional SUSY field theories, nontrivial fixed points and string dynamics, Phys. Lett. B 388 (1996) 753 [hep-th/9608111] [INSPIRE].

[6] D.R. Morrison and N. Seiberg, Extremal transitions and five-dimensional supersymmetric field theories, Nucl. Phys. B 483 (1997) 229 [hep-th/9609070] [INSPIRE].

[7] K.A. Intriligator, D.R. Morrison and N. Seiberg, Five-dimensional supersymmetric gauge theories and degenerations of Calabi-Yau spaces, Nucl. Phys. B 497 (1997) 56 [hep-th/9702198] [INSPIRE].

[8] A.D. Shapere and C. Vafa, BPS structure of Argyres-Douglas superconformal theories, hep-th/9910182 [INSPIRE].

[9] K. Hori, H. Ooguri and C. Vafa, Non-Abelian conifold transitions and $N=4$ dualities in three-dimensions, Nucl. Phys. B 504 (1997) 147 [hep-th/9705220] [INSPIRE].

[10] C. Closset, S. Schäfer-Nameki and Y.-N. Wang, On SCFTs at canonical singularities. Part 1 , to appear.

[11] H. Hayashi, C. Lawrie and S. Schäfer-Nameki, Phases, flops and F-theory: SU(5) gauge theories, JHEP 10 (2013) 046 [arXiv: 1304.1678] [INSPIRE].

[12] H. Hayashi, C. Lawrie, D.R. Morrison and S. Schäfer-Nameki, Box graphs and singular fibers, JHEP 05 (2014) 048 [arXiv: 1402 .2653] [INSPIRE].

[13] M. Del Zotto, J.J. Heckman and D.R. Morrison, $6 D$ SCFTs and phases of $5 D$ theories, JHEP 09 (2017) 147 [arXiv:1703.02981] [INSPIRE].

[14] P. Jefferson, H.-C. Kim, C. Vafa and G. Zafrir, Towards classification of 5d SCFTs: single gauge node, arXiv:1705.05836 [INSPIRE].

[15] C. Closset, M. Del Zotto and V. Saxena, Five-dimensional SCFTs and gauge theory phases: an M-theory/type IIA perspective, SciPost Phys. 6 (2019) 052 [arXiv:1812.10451] [INSPIRE].

[16] P. Jefferson, S. Katz, H.-C. Kim and C. Vafa, On geometric classification of 5d SCFTs, JHEP 04 (2018) 103 [arXiv: 1801.04036] [INSPIRE].

[17] F. Apruzzi, L. Lin and C. Mayrhofer, Phases of $5 d$ SCFTs from M-/F-theory on non-flat fibrations, JHEP 05 (2019) 187 [arXiv:1811.12400] [INSPIRE].

[18] L. Bhardwaj and P. Jefferson, Classifying 5d SCFTs via 6d SCFTs: rank one, JHEP 07 (2019) 178 [Addendum ibid. 01 (2020) 153] [arXiv:1809.01650] [INSPIRE]. 
[19] L. Bhardwaj and P. Jefferson, Classifying 5d SCFTs via 6d SCFTs: arbitrary rank, JHEP 10 (2019) 282 [arXiv: 1811.10616] [INSPIRE].

[20] F. Apruzzi, C. Lawrie, L. Lin, S. Schäfer-Nameki and Y.-N. Wang, 5d superconformal field theories and graphs, Phys. Lett. B 800 (2020) 135077 [arXiv:1906.11820] [INSPIRE].

[21] F. Apruzzi, C. Lawrie, L. Lin, S. Schäfer-Nameki and Y.-N. Wang, Fibers add flavor. Part I. Classification of 5d SCFTs, flavor symmetries and BPS states, JHEP 11 (2019) 068 [arXiv: 1907.05404] [INSPIRE].

[22] F. Apruzzi, C. Lawrie, L. Lin, S. Schäfer-Nameki and Y.-N. Wang, Fibers add flavor. Part II. 5d SCFTs, gauge theories, and dualities, JHEP 03 (2020) 052 [arXiv:1909.09128] [INSPIRE].

[23] L. Bhardwaj, On the classification of 5d SCFTs, JHEP 09 (2020) 007 [arXiv:1909.09635] [INSPIRE].

[24] F. Apruzzi, S. Schäfer-Nameki and Y.-N. Wang, 5d SCFTs from decoupling and gluing, JHEP 08 (2020) 153 [arXiv: 1912.04264] [INSPIRE].

[25] L. Bhardwaj, P. Jefferson, H.-C. Kim, H.-C. Tarazi and C. Vafa, Twisted circle compactifications of $6 d$ SCFTs, JHEP 12 (2020) 151 [arXiv:1909.11666] [INSPIRE].

[26] L. Bhardwaj, Do all 5d SCFTs descend from 6d SCFTs?, arXiv:1912.00025 [INSPIRE].

[27] J. Eckhard, S. Schäfer-Nameki and Y.-N. Wang, Trifectas for $T_{N}$ in 5d, JHEP 07 (2020) 199 [arXiv: 2004.15007] [INSPIRE].

[28] L. Bhardwaj, More 5d KK theories, arXiv:2005.01722 [INSPIRE].

[29] O. Aharony, A. Hanany and B. Kol, Webs of $(p, q)$ five-branes, five-dimensional field theories and grid diagrams, JHEP 01 (1998) 002 [hep-th/9710116] [INSPIRE].

[30] S. Cabrera and A. Hanany, Quiver subtractions, JHEP 09 (2018) 008 [arXiv:1803.11205] [INSPIRE].

[31] S. Cabrera, A. Hanany and F. Yagi, Tropical geometry and five dimensional Higgs branches at infinite coupling, JHEP 01 (2019) 068 [arXiv:1810.01379] [INSPIRE].

[32] S. Cabrera, A. Hanany and M. Sperling, Magnetic quivers, Higgs branches, and $6 d$ $N=(1,0)$ theories, JHEP 06 (2019) 071 [Erratum ibid. 07 (2019) 137] [arXiv: 1904.12293] [INSPIRE].

[33] A. Bourget et al., The Higgs mechanism - Hasse diagrams for symplectic singularities, JHEP 01 (2020) 157 [arXiv: 1908. 04245] [inSPIRE].

[34] A. Bourget, S. Cabrera, J.F. Grimminger, A. Hanany and Z. Zhong, Brane webs and magnetic quivers for SQCD, JHEP 03 (2020) 176 [arXiv: 1909.00667] [INSPIRE].

[35] S. Cabrera, A. Hanany and M. Sperling, Magnetic quivers, Higgs branches, and $6 d$ $N=(1,0)$ theories - orthogonal and symplectic gauge groups, JHEP 02 (2020) 184 [arXiv: 1912.02773] [INSPIRE].

[36] J.F. Grimminger and A. Hanany, Hasse diagrams for $3 d N=4$ quiver gauge theories inversion and the full moduli space, JHEP 09 (2020) 159 [arXiv:2004.01675] [INSPIRE].

[37] A. Bourget, J.F. Grimminger, A. Hanany, M. Sperling, G. Zafrir and Z. Zhong, Magnetic quivers for rank 1 theories, JHEP 09 (2020) 189 [arXiv:2006.16994] [INSPIRE]. 
[38] A. Bourget, J.F. Grimminger, A. Hanany, M. Sperling and Z. Zhong, Magnetic quivers from brane webs with O5 planes, JHEP 07 (2020) 204 [arXiv: 2004.04082] [INSPIRE].

[39] S.S.-T. Yau and Y. Yu, Classification of 3-dimensional isolated rational hypersurface singularities with C*-action, Rocky Mountain J. Math. 35 (2005) 1795 [math. AG/0303302] [INSPIRE].

[40] I.C. Davenport and I.V. Melnikov, Landau-Ginzburg skeletons, JHEP 05 (2017) 050 [arXiv: 1608. 04259] [INSPIRE].

[41] V.I. Arnold, S.M. Gusein-Zade and A.N. Varchenko eds., Singularities of differentiable maps, volume 2: monodromy and asymptotics of integrals, Birkhäuser, Boston, MA, U.S.A. (1988).

[42] S. Cecotti, A. Neitzke and C. Vafa, R-twisting and $4 d / 2 d$ correspondences, arXiv: 1006.3435 [INSPIRE].

[43] D. Xie and S.-T. Yau, $4 d N=2$ SCFT and singularity theory part I: classification, arXiv:1510.01324 [INSPIRE].

[44] B. Chen, D. Xie, S.-T. Yau, S.S.T. Yau and H. Zuo, $4 d N=2$ SCFT and singularity theory. Part II: complete intersection, Adv. Theor. Math. Phys. 21 (2017) 121 [arXiv:1604.07843] [INSPIRE].

[45] Y. Wang, D. Xie, S.S.T. Yau and S.-T. Yau, $4 d N=2 S C F T$ from complete intersection singularity, Adv. Theor. Math. Phys. 21 (2017) 801 [arXiv:1606.06306] [InSPIRE].

[46] B. Chen, D. Xie, S.S.T. Yau, S.-T. Yau and H. Zuo, $4 d N=2$ SCFT and singularity theory. Part III: rigid singularity, Adv. Theor. Math. Phys. 22 (2018) 1885 [arXiv:1712.00464] [INSPIRE].

[47] D. Xie and S.-T. Yau, Three dimensional canonical singularity and five dimensional $N=1$ SCFT, JHEP 06 (2017) 134 [arXiv:1704.00799] [INSPIRE].

[48] K.A. Intriligator and N. Seiberg, Mirror symmetry in three-dimensional gauge theories, Phys. Lett. B 387 (1996) 513 [hep-th/9607207] [INSPIRE].

[49] S. Cremonesi, A. Hanany and A. Zaffaroni, Monopole operators and Hilbert series of Coulomb branches of $3 d N=4$ gauge theories, JHEP 01 (2014) 005 [arXiv:1309.2657] [INSPIRE].

[50] H. Nakajima, Towards a mathematical definition of Coulomb branches of 3-dimensional $N=4$ gauge theories, I, Adv. Theor. Math. Phys. 20 (2016) 595 [arXiv:1503.03676] [INSPIRE].

[51] M. Bullimore, T. Dimofte and D. Gaiotto, The Coulomb branch of $3 d N=4$ theories, Commun. Math. Phys. 354 (2017) 671 [arXiv:1503.04817] [InSPIRE].

[52] A. Braverman, M. Finkelberg and H. Nakajima, Towards a mathematical definition of Coulomb branches of 3-dimensional $N=4$ gauge theories, II, Adv. Theor. Math. Phys. 22 (2018) 1071 [arXiv: 1601.03586] [INSPIRE].

[53] P.C. Argyres, M. Lotito, Y. Lü and M. Martone, Geometric constraints on the space of $N=2$ SCFTs. Part II: construction of special Kähler geometries and RG flows, JHEP 02 (2018) 002 [arXiv: 1601.00011] [inSPIRE].

[54] M. Martone, Towards the classification of rank-r $N=2$ SCFTs. Part I. Twisted partition function and central charge formulae, JHEP 12 (2020) 021 [arXiv:2006.16255] [INSPIRE]. 
[55] P. Argyres and M. Martone, Construction and classification of Coulomb branch geometries, arXiv: 2003.04954 [INSPIRE].

[56] P.C. Argyres and M. Martone, Towards a classification of rank-r $N=2$ SCFTs. Part II. Special Kähler stratification of the Coulomb branch, JHEP 12 (2020) 022 [arXiv:2007.00012] [INSPIRE].

[57] P.C. Argyres, K. Maruyoshi and Y. Tachikawa, Quantum Higgs branches of isolated $N=2$ superconformal field theories, JHEP 10 (2012) 054 [arXiv: 1206.4700] [INSPIRE].

[58] M. Del Zotto and A. Hanany, Complete graphs, Hilbert series, and the Higgs branch of the $4 d N=2\left(A_{n}, A_{m}\right)$ SCFTs, Nucl. Phys. B 894 (2015) 439 [arXiv:1403.6523] [inSPIRE].

[59] M. Buican and T. Nishinaka, On the superconformal index of Argyres-Douglas theories, J. Phys. A 49 (2016) 015401 [arXiv: 1505.05884] [INSPIRE].

[60] J. Song, Superconformal indices of generalized Argyres-Douglas theories from $2 d$ TQFT, JHEP 02 (2016) 045 [arXiv:1509.06730] [INSPIRE].

[61] K. Maruyoshi and J. Song, Enhancement of supersymmetry via renormalization group flow and the superconformal index, Phys. Rev. Lett. 118 (2017) 151602 [arXiv:1606.05632] [INSPIRE].

[62] K. Maruyoshi and J. Song, $N=1$ deformations and $R G$ flows of $N=2$ SCFTs, JHEP 02 (2017) 075 [arXiv: 1607.04281] [InSPIRE].

[63] P. Agarwal, K. Maruyoshi and J. Song, $N=1$ deformations and $R G$ flows of $N=2$ SCFTs, part II: non-principal deformations, JHEP 12 (2016) 103 [Addendum ibid. 04 (2017) 113] [arXiv:1610.05311] [INSPIRE].

[64] J. Song, D. Xie and W. Yan, Vertex operator algebras of Argyres-Douglas theories from M5-branes, JHEP 12 (2017) 123 [arXiv:1706.01607] [INSPIRE].

[65] P. Agarwal, A. Sciarappa and J. Song, $N=1$ Lagrangians for generalized Argyres-Douglas theories, JHEP 10 (2017) 211 [arXiv:1707.04751] [INSPIRE].

[66] S. Benvenuti and S. Giacomelli, Lagrangians for generalized Argyres-Douglas theories, JHEP 10 (2017) 106 [arXiv:1707.05113] [INSPIRE].

[67] P. Agarwal, S. Lee and J. Song, Vanishing OPE coefficients in 4d $N=2$ SCFTs, JHEP 06 (2019) 102 [arXiv: 1812.04743] [InSPIRE].

[68] C. Beem, M. Lemos, P. Liendo, W. Peelaers, L. Rastelli and B.C. van Rees, Infinite chiral symmetry in four dimensions, Commun. Math. Phys. 336 (2015) 1359 [arXiv:1312.5344] [INSPIRE].

[69] C. Beem and L. Rastelli, Vertex operator algebras, Higgs branches, and modular differential equations, JHEP 08 (2018) 114 [arXiv:1707.07679] [INSPIRE].

[70] C. Beem, C. Meneghelli and L. Rastelli, Free field realizations from the Higgs branch, JHEP 09 (2019) 058 [arXiv: 1903.07624] [INSPIRE].

[71] C. Beem, C. Meneghelli, W. Peelaers and L. Rastelli, VOAs and rank-two instanton SCFTs, Commun. Math. Phys. 377 (2020) 2553 [arXiv:1907.08629] [InSPIRE].

[72] S. Gukov, C. Vafa and E. Witten, CFT's from Calabi-Yau four folds, Nucl. Phys. B 584 (2000) 69 [Erratum ibid. 608 (2001) 477] [hep-th/9906070] [INSPIRE].

[73] N. Nekrasov, Five dimensional gauge theories and relativistic integrable systems, Nucl. Phys. B 531 (1998) 323 [hep-th/9609219] [INSPIRE]. 
[74] A. Kapustin and M.J. Strassler, On mirror symmetry in three-dimensional Abelian gauge theories, JHEP 04 (1999) 021 [hep-th/9902033] [INSPIRE].

[75] E. Witten, $\mathrm{SL}(2, Z)$ action on three-dimensional conformal field theories with Abelian symmetry, in From fields to strings: circumnavigating theoretical physics. A conference in tribute to Ian Kogan, (2003), pg. 1173 [hep-th/0307041] [INSPIRE].

[76] G. Ferlito, A. Hanany, N. Mekareeya and G. Zafrir, 3d Coulomb branch and $5 d$ Higgs branch at infinite coupling, JHEP 07 (2018) 061 [arXiv: 1712.06604] [INSPIRE].

[77] A. Hanany and N. Mekareeya, The small $E_{8}$ instanton and the Kraft Procesi transition, JHEP 07 (2018) 098 [arXiv:1801.01129] [INSPIRE].

[78] M. van Beest, A. Bourget, J. Eckhard and S. Schäfer-Nameki, (Symplectic) leaves and (5d Higgs) branches in the poly(go)nesian tropical rain forest, JHEP 11 (2020) 124 [arXiv: 2008. 05577] [INSPIRE].

[79] I. García Etxebarria, B. Heidenreich and D. Regalado, IIB flux non-commutativity and the global structure of field theories, JHEP 10 (2019) 169 [arXiv:1908.08027] [INSPIRE].

[80] D.R. Morrison, S. Schäfer-Nameki and B. Willett, Higher-form symmetries in 5d, JHEP 09 (2020) 024 [arXiv: 2005.12296] [INSPIRE].

[81] F. Albertini, M. Del Zotto, I. García Etxebarria and S.S. Hosseini, Higher form symmetries and M-theory, JHEP 12 (2020) 203 [arXiv:2005.12831] [INSPIRE].

[82] M. Del Zotto, I. García Etxebarria and S.S. Hosseini, Higher form symmetries of Argyres-Douglas theories, JHEP 10 (2020) 056 [arXiv: 2007.15603] [INSPIRE].

[83] P.C. Argyres and M.R. Douglas, New phenomena in SU(3) supersymmetric gauge theory, Nucl. Phys. B 448 (1995) 93 [hep-th/9505062] [INSPIRE].

[84] P.C. Argyres, M.R. Plesser, N. Seiberg and E. Witten, New $N=2$ superconformal field theories in four-dimensions, Nucl. Phys. B 461 (1996) 71 [hep-th/9511154] [INSPIRE].

[85] J.A. Minahan and D. Nemeschansky, An $N=2$ superconformal fixed point with $E_{6}$ global symmetry, Nucl. Phys. B 482 (1996) 142 [hep-th/9608047] [INSPIRE].

[86] D. Xie, General Argyres-Douglas theory, JHEP 01 (2013) 100 [arXiv:1204.2270] [INSPIRE].

[87] P. Arras, A. Grassi and T. Weigand, Terminal singularities, Milnor numbers, and matter in F-theory, J. Geom. Phys. 123 (2018) 71 [arXiv:1612.05646] [INSPIRE].

[88] A. Grassi and T. Weigand, On topological invariants of algebraic threefolds with (Q-factorial) singularities, arXiv:1804.02424 [INSPIRE].

[89] P.C. Argyres and M. Martone, $4 d N=2$ theories with disconnected gauge groups, JHEP 03 (2017) 145 [arXiv:1611.08602] [InSPIRE].

[90] O. Aharony and Y. Tachikawa, $S$-folds and $4 d N=3$ superconformal field theories, JHEP 06 (2016) 044 [arXiv: 1602.08638] [INSPIRE].

[91] Y. Tachikawa, On gauging finite subgroups, SciPost Phys. 8 (2020) 015 [arXiv:1712.09542] [INSPIRE].

[92] F. Apruzzi, S. Giacomelli and S. Schäfer-Nameki, $4 d N=2$ S-folds, Phys. Rev. D 101 (2020) 106008 [arXiv: 2001.00533] [INSPIRE].

[93] A. Hanany and G. Zafrir, Discrete gauging in six dimensions, JHEP 07 (2018) 168 [arXiv: 1804.08857] [INSPIRE]. 
[94] A. Hanany and M. Sperling, Discrete quotients of 3-dimensional $N=4$ Coulomb branches via the cycle index, JHEP 08 (2018) 157 [arXiv:1807.02784] [INSPIRE].

[95] A. Hanany and A. Zajac, Discrete gauging in Coulomb branches of three dimensional $N=4$ supersymmetric gauge theories, JHEP 08 (2018) 158 [arXiv:1807.03221] [INSPIRE].

[96] C. Cordova, T.T. Dumitrescu and K. Intriligator, Deformations of superconformal theories, JHEP 11 (2016) 135 [arXiv:1602.01217] [INSPIRE].

[97] C.-M. Chang, 5d and 6d SCFTs have no weak coupling limit, JHEP 09 (2019) 016 [arXiv: 1810.04169] [INSPIRE].

[98] A.C. Cadavid, A. Ceresole, R. D'Auria and S. Ferrara, Eleven-dimensional supergravity compactified on Calabi-Yau threefolds, Phys. Lett. B 357 (1995) 76 [hep-th/9506144] [INSPIRE].

[99] M. Caibar, Minimal models of canonical singularities and their cohomology, Ph.D. thesis, University of Warwick, Warwick, U.K. (1999).

[100] M. Caibar, Minimal models of canonical 3-fold singularities and their Betti numbers, Int. Math. Res. Not. 2005 (2005) 1563.

[101] H. Ooguri and C. Vafa, Summing up D instantons, Phys. Rev. Lett. 77 (1996) 3296 [hep-th/9608079] [inSPIRE].

[102] A. Bourget, J.F. Grimminger, A. Hanany, R. Kalveks, M. Sperling and Z. Zhong, Magnetic lattices for orthosymplectic quivers, JHEP 12 (2020) 092 [arXiv:2007.04667] [INSPIRE].

[103] S. Katz, P. Mayr and C. Vafa, Mirror symmetry and exact solution of $4 D N=2$ gauge theories: 1, Adv. Theor. Math. Phys. 1 (1998) 53 [hep-th/9706110] [InSPIRE].

[104] M. Del Zotto, C. Vafa and D. Xie, Geometric engineering, mirror symmetry and $6 d_{(1,0)} \rightarrow 4 d_{(\mathcal{N}=2)}$, JHEP 11 (2015) 123 [arXiv: 1504.08348] [INSPIRE].

[105] Y. Wang and D. Xie, Classification of Argyres-Douglas theories from M5 branes, Phys. Rev. D 94 (2016) 065012 [arXiv: 1509.00847] [INSPIRE].

[106] A.D. Shapere and Y. Tachikawa, Central charges of $N=2$ superconformal field theories in four dimensions, JHEP 09 (2008) 109 [arXiv:0804.1957] [INSPIRE].

[107] N. Seiberg and E. Witten, Electric-magnetic duality, monopole condensation, and confinement in $N=2$ supersymmetric Yang-Mills theory, Nucl. Phys. B 426 (1994) 19 [Erratum ibid. 430 (1994) 485] [hep-th/9407087] [INSPIRE].

[108] U. Lindström, M. Roček and R. von Unge, Hyper-Kähler quotients and algebraic curves, JHEP 01 (2000) 022 [hep-th/9908082] [INSPIRE].

[109] B. de Wit, M. Roček and S. Vandoren, Hypermultiplets, hyper-Kähler cones and quaternion Kähler geometry, JHEP 02 (2001) 039 [hep-th/0101161] [INSPIRE].

[110] S. Alexandrov, D. Persson and B. Pioline, On the topology of the hypermultiplet moduli space in type-II/CY string vacua, Phys. Rev. D 83 (2011) 026001 [arXiv:1009.3026] [INSPIRE].

[111] H. Shimizu, Y. Tachikawa and G. Zafrir, Anomaly matching on the Higgs branch, JHEP 12 (2017) 127 [arXiv: 1703.01013] [INSPIRE].

[112] C.-M. Chang, M. Fluder, Y.-H. Lin and Y. Wang, Proving the 6d Cardy formula and matching global gravitational anomalies, arXiv:1910.10151 [INSPIRE]. 
[113] J. Milnor, Singular points of complex hypersurfaces, Princeton University Press, Princeton, NJ, U.S.A. (1968).

[114] P. Candelas and X.C. de la Ossa, Comments on conifolds, Nucl. Phys. B 342 (1990) 246 [INSPIRE].

[115] J. Sparks, Sasaki-Einstein manifolds, Surveys Diff. Geom. 16 (2011) 265 [arXiv: 1004.2461] [INSPIRE].

[116] R.C. Randell, The homology of generalized Brieskorn manifolds, Topology 14 (1975) 347.

[117] P. Orlik, On the homology of weighted homogeneous manifolds, in Proceedings of the second conference on compact transformation groups, H.T. Ku, L.N. Mann, J.L. Sicks and J.C. Su eds., Springer, Berlin, Heidelberg, Germany (1972), pg. 260.

[118] N. Seiberg and E. Witten, Gauge dynamics and compactification to three-dimensions, in Conference on the mathematical beauty of physics (in memory of C. Itzykson), (1996), pg. 333 [hep-th/9607163] [INSPIRE].

[119] D. Gaiotto, G.W. Moore and A. Neitzke, Four-dimensional wall-crossing via three-dimensional field theory, Commun. Math. Phys. 299 (2010) 163 [arXiv:0807.4723] [INSPIRE].

[120] M. Buican and T. Nishinaka, Argyres-Douglas theories, $S^{1}$ reductions, and topological symmetries, J. Phys. A 49 (2016) 045401 [arXiv: 1505.06205] [INSPIRE].

[121] F. Benini, S. Benvenuti and Y. Tachikawa, Webs of five-branes and $N=2$ superconformal field theories, JHEP 09 (2009) 052 [arXiv:0906.0359] [INSPIRE].

[122] F. Benini, Y. Tachikawa and D. Xie, Mirrors of 3d Sicilian theories, JHEP 09 (2010) 063 [arXiv: 1007.0992] [INSPIRE].

[123] A. Dey, Three dimensional mirror symmetry beyond ADE quivers and Argyres-Douglas theories, arXiv:2004.09738 [INSPIRE].

[124] D. Gaiotto, A. Kapustin, N. Seiberg and B. Willett, Generalized global symmetries, JHEP 02 (2015) 172 [arXiv: 1412.5148] [INSPIRE].

[125] O. Aharony, N. Seiberg and Y. Tachikawa, Reading between the lines of four-dimensional gauge theories, JHEP 08 (2013) 115 [arXiv:1305.0318] [INSPIRE].

[126] D.S. Freed, G.W. Moore and G. Segal, Heisenberg groups and noncommutative fluxes, Annals Phys. 322 (2007) 236 [hep-th/0605200] [INSPIRE].

[127] D. Gaiotto and H.-C. Kim, Duality walls and defects in $5 d N=1$ theories, JHEP 01 (2017) 019 [arXiv: 1506.03871] [inSPIRE].

[128] C. van Coevering, Examples of asymptotically conical Ricci-flat Kähler manifolds, Math. Z. 267 (2011) 465 [INSPIRE].

[129] L. Bhardwaj and Y. Tachikawa, Classification of $4 d N=2$ gauge theories, JHEP 12 (2013) 100 [arXiv: 1309.5160] [INSPIRE].

[130] M.R. Douglas and G.W. Moore, D-branes, quivers, and ALE instantons, hep-th/9603167 [INSPIRE].

[131] C. Lawrie and S. Schäfer-Nameki, The Tate form on steroids: resolution and higher codimension fibers, JHEP 04 (2013) 061 [arXiv: 1212.2949] [INSPIRE]. 
[132] V. Saxena, Rank-two 5d SCFTs from M-theory at isolated toric singularities: a systematic study, JHEP 04 (2020) 198 [arXiv:1911.09574] [INSPIRE].

[133] S. Cremonesi, G. Ferlito, A. Hanany and N. Mekareeya, Coulomb branch and the moduli space of instantons, JHEP 12 (2014) 103 [arXiv: 1408.6835] [INSPIRE].

[134] H. Hayashi, S.-S. Kim, K. Lee and F. Yagi, Dualities and 5-brane webs for $5 d$ rank 2 SCFTs, JHEP 12 (2018) 016 [arXiv: 1806. 10569] [INSPIRE].

[135] H. Hayashi, S.-S. Kim, K. Lee and F. Yagi, Complete prepotential for $5 d N=1$ superconformal field theories, JHEP 02 (2020) 074 [arXiv:1912.10301] [INSPIRE].

[136] A. Hanany and R. Kalveks, Quiver theories and formulae for nilpotent orbits of exceptional algebras, JHEP 11 (2017) 126 [arXiv:1709.05818] [INSPIRE].

[137] C. Closset, S. Giacomelli, S. Schäfer-Nameki and Y.-N. Wang, 5d and 4d SCFTs: canonical singularities, trinions and S-dualities, arXiv:2012.12827 [INSPIRE].

[138] V.I. Arnold, Critical points of smooth functions and their normal forms, Russ. Math. Surv. 30 (1975) 1.

[139] S. Cecotti and M. Del Zotto, On Arnold's 14 'exceptional' $N=2$ superconformal gauge theories, JHEP 10 (2011) 099 [arXiv:1107.5747] [INSPIRE].

[140] S. Benvenuti, A tale of exceptional 3d dualities, JHEP 03 (2019) 125 [arXiv:1809.03925] [INSPIRE].

[141] M. Dedushenko and Y. Wang, $4 d / 2 d \rightarrow 3 d / 1 d$ : a song of protected operator algebras, arXiv: 1912.01006 [INSPIRE].

[142] D. Nanopoulos and D. Xie, More three dimensional mirror pairs, JHEP 05 (2011) 071 [arXiv:1011.1911] [INSPIRE].

[143] D. Gaiotto, $N=2$ dualities, JHEP 08 (2012) 034 [arXiv:0904.2715] [INSPIRE].

[144] S. Cecotti, M. Del Zotto and S. Giacomelli, More on the $N=2$ superconformal systems of type $D_{p}(G)$, JHEP 04 (2013) 153 [arXiv: 1303.3149] [INSPIRE].

[145] E. Beratto, S. Giacomelli, N. Mekareeya and M. Sacchi, 3d mirrors of the circle reduction of twisted $A_{2 N}$ theories of class S, JHEP 09 (2020) 161 [arXiv:2007.05019] [INSPIRE].

[146] M. Buican, S. Giacomelli, T. Nishinaka and C. Papageorgakis, Argyres-Douglas theories and S-duality, JHEP 02 (2015) 185 [arXiv:1411.6026] [INSPIRE].

[147] D. Gaiotto and E. Witten, S-duality of boundary conditions in $N=4$ super Yang-Mills theory, Adv. Theor. Math. Phys. 13 (2009) 721 [arXiv:0807.3720] [InSPIRE].

[148] D. Gaiotto and S.S. Razamat, Exceptional indices, JHEP 05 (2012) 145 [arXiv:1203.5517] [INSPIRE].

[149] C. Closset and M. Del Zotto, On 5d SCFTs and their BPS quivers. Part I: B-branes and brane tilings, arXiv:1912.13502 [INSPIRE].

[150] I.R. Klebanov and E. Witten, Superconformal field theory on three-branes at a Calabi-Yau singularity, Nucl. Phys. B 536 (1998) 199 [hep-th/9807080] [INSPIRE].

[151] M. Fazzi and A. Tomasiello, Holography, matrix factorizations and K-stability, JHEP 05 (2020) 119 [arXiv: 1906. 08272] [InSPIRE].

[152] A. Hanany and R. Kalveks, Quiver theories for moduli spaces of classical group nilpotent orbits, JHEP 06 (2016) 130 [arXiv: 1601.04020] [INSPIRE]. 
[153] P. Argyres, M. Lotito, Y. Lü and M. Martone, Geometric constraints on the space of $N=2$ SCFTs. Part III: enhanced Coulomb branches and central charges, JHEP 02 (2018) 003 [arXiv: 1609.04404] [INSPIRE].

[154] K. Altmann, The versal deformation of an isolated toric Gorenstein singularity, alg-geom/9403004.

[155] J.P. Gauntlett, D. Martelli, J.F. Sparks and D. Waldram, A new infinite class of Sasaki-Einstein manifolds, Adv. Theor. Math. Phys. 8 (2004) 987 [hep-th/0403038] [INSPIRE].

[156] V. Borokhov, A. Kapustin and X.-K. Wu, Monopole operators and mirror symmetry in three-dimensions, JHEP 12 (2002) 044 [hep-th/0207074] [INSPIRE].

[157] S. Cremonesi, 3d supersymmetric gauge theories and Hilbert series, Proc. Symp. Pure Math. 98 (2018) 21 [arXiv:1701.00641] [INSPIRE].

[158] S. Benvenuti, B. Feng, A. Hanany and Y.-H. He, Counting BPS operators in gauge theories: quivers, Syzygies and Plethystics, JHEP 11 (2007) 050 [hep-th/0608050] [INSPIRE].

[159] C. Closset and H. Kim, Comments on twisted indices in 3d supersymmetric gauge theories, JHEP 08 (2016) 059 [arXiv: 1605.06531] [INSPIRE].

[160] S.S. Razamat and B. Willett, Down the rabbit hole with theories of class S, JHEP 10 (2014) 099 [arXiv: 1403.6107] [INSPIRE].

[161] M. van Beest, A. Bourget, J. Eckhard and S. Schäfer-Nameki, (Decoupling) trees in the tropical rain forest, to appear. 